\title{
Exploring Halo Substructure with Giant Stars VIII: The Extended Structure of the Sculptor Dwarf Spheroidal Galaxy
}

\author{
Kyle B. Westfall ${ }^{1,2}$, Steven R. Majewski ${ }^{1,3,4}$, James C. Ostheimer ${ }^{1,5}$, Peter M. \\ Frinchaboy ${ }^{1,3}$, William E. Kunkel ${ }^{3,6}$, Richard J. Patterson ${ }^{1}$, and Robert Link ${ }^{1,7}$ \\ westfall@astro.wisc.edu, srm4n@virginia.edu, jco9w@alumni.virginia.edu, \\ pmf8b@virginia.edu, kunkel@jeito.lco.cl, ricky@virginia.edu, \\ robert.link@ngc.com
}

\begin{abstract}
We explore the spatial distribution of stars in the Sculptor dwarf spheroidal (dSph) galaxy over an area of $7.82 \mathrm{deg}^{2}$, including coverage of the central region but extending mostly south and east of the dSph core. Two methods are used to identify stars that are most likely associated with the dSph, and these filtered samples of stars are used to map its spatial structure. First, following the method of previous contributions in this series, we utilize Washington $M, T_{2}+D D O 51$ photometry to identify red giant branch (RGB) star candidates with approximately the same distance and metallicity as the Sculptor dSph. Second, a prominent blue horizontal branch (BHB) population provides a fairly populous and pure sample of Sculptor stars having broadband colors unlike the bulk of the Galactic field star population. A spectroscopically observed subset of Sculptor candidate stars (147 total stars: $\sim 5 \%$ of all Sculptor candidates, $\sim 10 \%$ of Sculptor giant candidates) yields a systemic heliocentric velocity for the system
\end{abstract}

\footnotetext{
${ }^{1}$ University of Virginia, Department of Astronomy, Charlottesville, VA 22903-0818

${ }^{2}$ Department of Astronomy, University of Wisconsin-Madison, 475 N. Charter St., Madison, WI 53706

${ }^{3}$ Visiting Astronomer, Cerro Tololo Inter-American Observatory, National Optical Astronomy Observatory, which is operated by the Association of Universities for Research in Astronomy, Inc., under cooperative agreement with the National Science Foundation.

${ }^{4}$ Visiting Associate, The Observatories of the Carnegie Institution of Washington, 813 Santa Barbara Street, Pasadena, CA 91101.

${ }^{5}$ Present address: 1810 Kalorama Rd., NW, \#A3, Washington, D.C. 20009.

${ }^{6}$ Las Campanas Observatory, Carnegie Institution of Washington, Casilla 601, La Serena, Chile.

${ }^{7}$ Present address: Northrop Grumman Information Technology - TASC, 4801 Stonecroft Blvd., Chantilly, VA 20151.
} 
of $v_{\text {hel }}=110.43 \pm 0.79 \mathrm{~km} \mathrm{~s}^{-1}$, in good agreement with previous studies. We also find a global velocity dispersion of $\sigma_{v}=8.8 \pm 0.6 \mathrm{~km} \mathrm{~s}^{-1}$ with slight indications of a rise in the velocity dispersion past $\sim 0.4 r_{\text {lim }}$. These spectra also provide a check on the reliability of our candidate Sculptor giant sample to $M \sim 19$ : 94\% of the photometrically-selected Sculptor giant star candidates with follow-up spectroscopy are found to be kinematically associated with Sculptor, while four out of ten stars outside of our Sculptor giant star selection criteria that we tested spectroscopically appear to be velocity members of Sculptor. These percentages are in agreement with results for an additional 22 Sculptor field stars with radial velocities in the literature. All available velocities show that our methodology for picking Sculptor giants is both reliable and conservative. Thus, these giant star samples should provide a reliable means to explore the structure of the Sculptor dSph. Nevertheless, considerable care has been taken to assess the level of background contamination in our photometric sample to ensure an accurately derived density profile of the Sculptor dSph to large radii. Multiple background assessments verify that we detect a considerable stellar density of Sculptor stars to the limits of our main survey area for both the RGB and BHB candidate samples. While we find that a King profile of limiting radius $r_{\text {lim }}=79.6$ fits the density profile of Sculptor well to $\sim 60^{\prime}$, beyond this, we identify a "break" in the profile and a clearly detected population of Sculptor stars following a $\Sigma \propto r^{-2}$ decline to more than $2 r_{\text {lim. }}$. This break population must signify either the presence of an extremely broad distribution of bound "halo stars" around the Sculptor dSph, or the presence of unbound tidal debris. If the latter is true, we determine a fractional mass-loss rate of approximately $0.042 \mathrm{Gyr}^{-1}$ for the Sculptor dSph. Additional support for the notion that there is tidal disruption comes from the two-dimensional distribution of our Sculptor candidate stars: Both the RGB and BHB samples show increasingly elongated isodensity contours with radius that point to an apparent stretching reminiscent of what is seen in models of disrupting satellite galaxies. Finally, we find that RGB stars that are more likely to be metal-poor (based on their color and magnitude) are significantly less centrally concentrated and therefore constitute the primary contributing stellar population to the likely tidally-stripped parts of the dSph.

Subject headings: galaxies: dwarf — galaxies: fundamental parameters — galaxies: halos - galaxies: individual (Sculptor dSph) — galaxies: photometry galaxies: stellar content — galaxies: structure — Local Group 


\section{INTRODUCTION}

Dwarf spheroidal (dSph) galaxies were immediately recognized as mysterious stellar systems upon Shapley's discovery of the first example of this galaxy class, "a stellar system of a new type", in the constellation Sculptor (Shapley 1938a,b, 1939). As Shapley (1938b) pointed out after his discovery of a second example in the Fornax constellation soon afterward, the dSph galaxies "have some properties in common with globular clusters, others with spheroidal galaxies, and still others (nearness and complete resolution into stars) with the Magellanic Clouds." The uniqueness of these systems was further born out by Thackeray's study of Sculptor's variable star population, which indicated a "physical dissimilarity" between this new stellar system and a globular cluster (Thackeray 1950). However, to Shapley the density profile was the most remarkable aspect of these diffuse, low-surface-brightness "clusters"; indeed, he points out that had not the discovery plate been abnormally sensitive and taken during sky conditions that were also above average, "the Sculptor cluster would not have been found."

It is fair to say that the density distribution (stellar number and mass density) of dSph galaxies remains one of the most perplexing aspects of these systems. When the radial light profiles are converted to a stellar mass density and compared to that suggested by the internal dynamics of stars in these systems, very large dark matter contents are inferred (Faber \& Lin 1983; Aaronson 1983; Hargreaves et al. 1994; Irwin \& Hatzidimitriou 1995, see Mateo 1998 for a summary). From these measurements, the very low mass dSph galaxies make up the high end of the current mass-to-light $(M / L)$ scale for distinct stellar systems, with members having total $M / L$ 's approaching 100 (cf. Mateo 1998), or even exceeding that value by almost an order of magnitude (Kleyna et al. 2001, 2002). Even on the low end of this scale, with an apparent total $M / L \sim 10$ (Armandroff \& Da Costa 1986; Queloz, Dubath \& Pasquini 1995; Irwin \& Hatzidimitriou 1995), the Sculptor dSph is extraordinarily dominated by dark matter for such a low-mass stellar system $\left(\sim 10^{7} M_{\odot}\right)$.

The physical extents of dSph galaxies also remain uncertain, but have become a fulcrum on which recent debate regarding the true dark matter content of dSph's lie. The question is not only whether all of the light has been fully accounted for (e.g., Odenkirchen et al. 2001; Palma et al. 2003) but whether the increasingly large extensions of the radial profiles found by various observers on well-studied dSph examples are clues to something untoward in our basic understanding of the true dark matter content/distribution within, and the dynamical state of, these systems (e.g., Kuhn 1993; Kroupa 1997; Gómez-Flechoso 1998; Muñoz et al. 2005a,b; Majewski et al. 2005b).

The situation for the Sculptor dSph is more or less representative of the history of attempting to understand the dSph galaxy class as a whole. On shallow images including 
only the top two magnitudes of its luminosity function, Shapley (1938a) originally showed the radius of the Sculptor system to be at least 40', but with evidence for stars extending to as much as a degree from the center. Extending Shapley's starcount work, Hodge (1961, hereafter H61) conducted a $2.2 \mathrm{deg}^{2}$ survey using more plates of slightly shallower depth. He determined a limiting radius of $46^{\prime} \pm 3^{\prime}$ for Sculptor, which was close to Shapley's lower limit. More importantly, H61 found a "definite limiting radius" where the density of stars in Sculptor apparently reaches zero. Under the assumption of $M / L \sim 2$, this radius was consistent with expectations for a system that is tidally limited by the Milky Way (MW). Unfortunately, density profiles that drop sharply to zero density can be artificially created by overestimates of a subtracted background level, and it would seem that Hodge's survey area was too limited to contain true "background" regions free of Sculptor stars (as we show later in this paper).

Subsequent studies of the distribution of Sculptor's substantial RR Lyrae population found it to be much more extended than the quoted H61 tidal limit of the galaxy: van Agt (1973) states, "Variable stars have been traced in the Sculptor system out to distances of well over 60'." Later Innanen \& Papp (1979) fit a King (1962) model to the distribution of RR Lyrae stars and found a King limiting radius $\left(r_{\text {lim }}\right)$ of $47^{\prime} .6$, in good agreement with the H61 measurement. However, Innanen \& Papp (1979) also found that likely $10 \%$ of Sculptor RR Lyraes are located outside this limiting radius (which they interpret as a tidal radius) and that they extend to $3^{\circ}$ from the center of the galaxy. The dynamical nature of individual stars (such as the above variable stars) or statistical stellar overdensities beyond the "limiting radii" of dSphs is quite contentious, but as a group these stars are often termed "extratidal", as was done by Innanen \& Papp (1979). While this is an often-adopted shorthand, note that (1) King models may not necessarily apply to dSph galaxies, which have longer relaxation timescales than the globular clusters for which the models were intended, and, therefore, (2) some models of dwarf spheroidal structure may include bound "extratidal" populations beyond a King profile. To allay confusion between the observed spatial position and the actual dynamical state of stars found beyond the King limiting radius, we limit use of the expression "extratidal" here, preferring instead the term "break population", which refers to a change in the slope of the radial density law that these stars create.

The results from Innanen \& Papp (1979) led others to reevaluate the spatial distribution of the Sculptor dSph. Demers, Kunkel \& Krauter (1980) obtained photographic data $\sim 4$ magnitudes deeper than that presented by $\mathrm{H} 61$ and found a limiting radius between $75^{\prime}$ and $120^{\prime}$; however, Demers et al. note that this value is highly dependent on the adopted background. They could obtain the H61 limiting radius after subtracting an excessively large background level (an effect no doubt confounding the original H61 study); but, they could also obtain a limiting radius that was "essentially infinite" by adopting their lowest 
background estimate. In a study that included multiple UK Schmidt plates that reached much greater angular distances from the center of Sculptor and $\sim 2$ magnitudes deeper than the H61 data, Eskridge (1988b) noted a similar sensitivity of his results to the adopted background level. In order to force the King model to fit all points to the extent of his survey, the adopted background had to be $3 \sigma$ higher than the calculated background, which forced all points with $r>60^{\prime}$ below the background level. This study ultimately identified a best-fit King model with $r_{\text {lim }}=95^{\prime}$ but noted the inability of this fitted profile to fully describe the radial distribution of Sculptor. Irwin \& Hatzidimitriou (1995, hereafter IH95) used one UK Schmidt plate of similar depth to those of Eskridge (1988b) and found $r_{\lim }=76.5$. CCD data going approximately one magnitude deeper but over slightly less than half the area of IH95 was recently used by Walcher et al. (2003, hereafter W03) who determined, however, $r_{\lim }=44^{\prime}$ using the theoretical King (1966) profile.

In three of the four most recent studies, not only has the derived King profile limiting radius grown, but the density of the presumed Sculptor stars near and beyond this radius has been found to be significantly $(\gtrsim 2 \sigma)$ in excess of densities predicted by the King model. In fact, the starcounts from Eskridge (1988b) show the overdense, "extratidal" population represents roughly $13 \%$ of the whole of Sculptor, in agreement with measurements from the RR Lyrae distribution (Innanen \& Papp 1979). Moreover, W03 have claimed not only the existence of a break population but also a hint of extratidal arms extending from Sculptor to the northwest and southeast. We address this and other previous results in comparison with our own in more detail in $\S 5$ and $\S 6$.

An overdensity at large radii is not exclusive to the Sculptor dSph. In their study, which included eight of the now ten known local dSphs, IH95 comment, "There is a noticeable tendency for most of the dSphs to show an excess of stars, with respect to the best-fitting King model, at large radii." IH95 state that this excess density is not likely caused by incorrect background determinations. As previously discovered by Demers, Kunkel \& Krauter (1980) and Eskridge (1988b), IH95 find an unreasonably large background estimate would have to be adopted to eliminate the excess density in the case of every $d S p h$. Subsequently, break populations were reported in other studies for Carina (Kuhn, Smith \& Hawley 1996; Majewski et al. 2000a, 2005a), Ursa Minor (Kocevski \& Kuhn 2000; Martínez-Delgado et al. 2001; Palma et al. 2003), Leo I (Sohn et al. 2005), Draco (Wilkinson et al. 2004), and possibly Sextans (Gould et al. 1992). Of course, the Sagittarius dSph (Sgr) (Ibata et al. 1995 ) is the clearest indicator that at least one satellite galaxy of the MW is experiencing tidally-induced mass loss, which contributes a break population to its radial profile. This dSph has now been shown to have extratidal debris wrapping more than $360^{\circ}$ around the Galaxy (e.g., Ibata et al. 2001; Majewski et al. 2003). 
Unfortunately, the existence or cause of the break populations in other dSphs are not as clear as in the Sgr system. For example, whether a break population exists around the Draco dSph has been controversial and highlights the importance of careful studies in the low-density regimes of the radial density profile. Smith, Kuhn \& Hawley (1997), Kocevski \& Kuhn (2000), Piatek et al. (2001), and Wilkinson et al. (2004) all report evidence for stars beyond the nominal Draco limiting radius. However, using Sloan Digital Sky Survey data, Odenkirchen et al. (2001) find no tidal extensions and no evidence of a break in the radial density profile, a result also found by Piatek et al. (2002) (who apparently reverse the previous conclusions of Piatek et al. 2001). In addition, both Odenkirchen et al. (2001) and Aparicio, Carrera, \& Martínez-Delgado (2001) find that the radial distribution of Draco is better fit by an exponential profile with no real limiting radius as prescribed by the King (1962) profile, whereas a change in the slope (from a "break population") of the Draco density profile is obvious in the data shown by Wilkinson et al. (2004). Similar controversy has been raised in the case of the Carina dSph, for which IH95, Kuhn et al. (1996) and Majewski et al. (2000a) all claim a break population but for which W03 find no break population and Morrison et al. (2001) question the Majewski et al. (2000a) result. A thorough reanalysis of the debate surrounding the existence of a break population in Carina is given in Majewski et al. (2005a, hereafter Paper VI) and further proof that a Carina break population exists is given in Muñoz et al. (2005b, hereafter Mu05b).

These case histories suggest that the state of the dSph observational record remains unsettled (or, at least, not universally accepted), and this empirical ambiguity forestalls any meaningful (or, at least, complete) interpretation of the physical state of these systems. As discussed above, the observational difficulty lies in a proper accounting for the lowdensity dSph regions against the usually overwhelming contamination by foreground field stars and background galaxies. As we have seen, the physical extent of the Sculptor dSph has apparently grown with deeper and/or more accurate studies conducted over larger areas. To improve the contrast of the true dSph members over the contaminating background (hereafter the "signal-to-background" ratio or $S / B$ ), survey approaches have progressed from the early simple star count analyses to searches in selectively tuned subregions of color-magnitude space (e.g., Kuhn et al. 1996; Grillmair et al. 1995; Odenkirchen et al. 2001; Rockosi et al. 2002; Piatek et al. 2002). For the dSphs, such studies rely on deep photometry reaching to dSph-rich parts of the CMD, such as the main sequence turn-off (MSTO), to boost the contributed dSph signal. However, to employ such a technique in the case of an expansive and distant system like Sculptor, which in some previous estimates has an $r_{\text {lim }}$ as large as 1.5 and which has a MSTO near $V \sim 23.5$, one anticipates needing a substantial amount of imaging ( say $\sim 16 \mathrm{deg}^{2}$ ) on 4-m class telescopes or larger to confidently reach at least twice the King limiting radius in all directions. Even attempting such an experiment on only a 
fraction of this area with the currently largest mosaic CCD cameras is a daunting prospect.

In this series of papers we have taken an alternative approach to high $S / B$ mapping of low-density, extended regions of Galactic satellites by concentrating instead on reducing the background in the problem. Our technique employs more easily obtainable, relatively shallow imaging in the Washington $M, T_{2}+D D O 51$ filter system to isolate (typically low-metallicity) giant stars associated with the $\mathrm{dSph}$ from the primary field contaminant, foreground dwarf stars from the more metal-rich disk. This methodology goes a long way toward the limit of zero background, so that we are left with relatively pure samples of bona fide dSph-associated giant stars. A benefit of this approach is that we identify the very stars needed for practical follow-up, spectroscopic analysis. So far in this series we have presented the methodology (Majewski et al. 2000b, hereafter Paper I) and applied it to determine the distributions of giant stars in the Carina, Ursa Minor, Draco and Leo I dSphs (Majewski et al. 2000a; Palma et al. 2003; Muñoz et al. 2005a; Sohn et al. 2005, hereafter Paper II, Paper IV, Paper IX and Paper X). This technique has also been used to study the M31 dSph galaxies And I, And II and And III (Ostheimer 2002). A preliminary report on our survey of Sculptor has been given in Westfall et al. (2000). We have given a report of preliminary results on these and other dSphs we are currently investigating, including Sculptor, in Majewski et al. (2002) and Majewski (2003).

Since those summary reports and Westfall et al. (2000), we have extended the radial coverage of our Washington+DDO51 survey around Sculptor, improved the critical assessment of the residual background noise, and included a parallel analysis using Sculptor's horizontal branch stars. We report here evidence for an extended, King profile break population of stars around the Sculptor dSph out to at least two limiting radii. These results are found with independent analyses of both blue horizontal branch (BHB) and red giant branch (RGB) star tracers. These photometric mappings are backed up with spectroscopic verification of radial velocity membership for a small subsample of selected Sculptor star candidates (§4). Our findings here strengthen the claim that at least some Galactic dSphs may be experiencing non-negligible stellar mass loss, most likely tidally induced. Thus, they provide important constraints applicable to the ultimate goals of our overall, long-term project, which are to (1) understand satellite disruption within the context of the hierarchical build-up of the Galactic halo as predicted by Cold Dark Matter models (e.g., Press \& Schechter 1974; Kauffmann \& White 1993; Lacey \& Cole 1993; Navarro, Frenk \& White 1996, 1997; Bullock, Kravtsov \& Weinberg 2001), and explored in simulations of halo substructure (e.g., Johnston 1998; Bullock et al. 2001; Harding et al. 2001; Johnston, Spergel, \& Haydn 2002; Hayashi et al. 2003), and (2) determine the dynamical conditions of dSph galaxies within the context of their inferred large $M / L$ values (e.g., Kuhn 1993; Oh, Lin, \& Aarseth 1995; Piatek \& Pryor 1995; Kroupa 1997; Gómez-Flechoso 1998, ; Mu05b). 
We present the photometric data and subsequent reduction methods in $\S 2$. In $\S 3$, we describe our selection criteria and its application to our photometric survey. Spectroscopic measurements are presented in $\S 4$ that provide both a limited exploration of the dynamics of the dSph and an estimate of the accuracy of our photometric selection technique. Spatial analyses of our photometrically-selected Sculptor candidate samples are given in $\S 5$ and we conclude with some discussion of our results in $\S 6$.

\section{PHOTOMETRY}

A mosaic of the Sculptor dSph was created via CCD imaging undertaken over the course of six observing runs at the Swope 1-m telescope located at the Las Campanas Observatory. Table 1 lists the UT dates of these observations, average seeing estimates, lunar illumination fraction, the total number of Sculptor fields observed $\left(N_{\text {fields }}\right)$, and the number of photometric fields $\left(N_{\text {phot }}\right)$. Each individual pointing in the survey area was observed once in the Washington $M$ and $T_{2}$ filters and twice in the DDO51 filter with nominal exposure times of 240, 240, and 840 seconds, respectively. Observations before the year 2001 were taken with the $2048 \times 2048$ SITe \#1 CCD chip while those made after this were taken with the $2048 \times 4096$ SITe \#3 CCD chip. The SITe \#3 chip has a hot pixel at $(x, y) \approx(808,3158)$ and, as a consequence, is mounted in the dewar so that the good three quarters of the chip is centered, while the poorer fourth quarter is somewhat vignetted. In order to maximize sky coverage, we have included the full, unvignetted portion of the SITe \#3 chip frames in our reduction, which happens to include the hot pixel ${ }^{1}$. The total sky coverage is approximately $0.16 \operatorname{deg}^{2}$ (0'.697 per pixel, Cudworth \& Rees 1991) for the SITe \#1 chip and 0.10 deg $^{2}$ (0'435 per pixel) for the SITe \#3 chip.

Individual pointings were arranged in a grid pattern with $\sim 3^{\prime}$ overlaps in order to get contiguous coverage of the Sculptor field yet allow for calibration of non-photometric frames by overlapping, adjacent photometric ones in a boot-strap process (see below). With a previously determined limiting radius of $r_{\text {lim }}=76$ '.5 (IH95), coverage of the entire galaxy

\footnotetext{
${ }^{1}$ Due to its use in the latter stages of our observing program, fields completed with the SITe \#3 chip are primarily at large distances from the dSph core or are used to provide photometric anchors for calibration. Inclusion of the hot pixel area will, in this region of the CCD field: (1) increase the photometric error of the objects detected, (2) hinder the detection of fainter sources, which effectively forces a brighter magnitude limit, and (3) cause an underestimation of the stellar density. All of these effects are largely mitigated by applied limits in allowable photometric error (discussed later) and, in the end, only a small portion $(\lesssim 5 \%)$ of the total survey area is affected. In fact, the surface density profile is more affected by having unobserved regions in the elliptical annuli at large radii than by including the area affected by the hot pixel.
} 
to significantly large radii (e.g., $1.5 r_{\text {lim }}-2 r_{\text {lim }}$ ) would be very ambitious when one is limited to $0.1-0.16 \mathrm{deg}^{2}$ per pointing. Therefore, to explore the nature of the Sculptor radial profile to large radii, our survey strategy focused on covering an area of approximately $1.28 \mathrm{deg}^{2}$ at the dSph center, but with the majority of attention extended to $\sim 2 r_{\text {lim }}$ toward the east and south. Even this "lop-sided" coverage over $7.82 \mathrm{deg}^{2}$ required nearly 100 separate pointings — 59 from the SITe \#1 chip and 37 from the SITe \#3 chip — and nearly 400 individual CCD frames. The survey includes four background "control" pointings taken $5^{\circ}$ from the center of the galaxy in each cardinal direction. These control fields provide important tests of the stellar background near the Sculptor dSph (§5.1).

Figure 1 shows the distribution of all photometered objects in both the full survey area including the four control pointings and the main part of the survey. Some pointings were later found not to be at their nominal positions due to pointing errors at the telescope. Subsequent maps in this paper will only show the inner regions though all analyses were performed on both the main survey and the control fields.

Basic image reductions used the imred.ccdred package in IRAF $^{2}$. Most nightly observations contained bias frames, dome flats, and sky flats for each of the three filters. Those nights without bias frames or dome flats were reduced using the most appropriate calibration frames from the nearest adjacent night. Those nights with sky flats and/or uncrowded object images with high backgrounds were combined and used to create illumination correction images. Final frames used for photometry had large-scale flux variations of at most $\epsilon_{f} \lesssim 2 \%$, with the vast majority having $\epsilon_{f} \lesssim 1 \%$, such that flat-fielding errors contribute a magnitude error of $\epsilon_{m} \lesssim 0.01$.

Point spread function(PSF)-fitted photometry of the object frames was done with the stand-alone version of DAOPHOT II (Stetson 1992). Magnitudes, magnitude errors, fitting errors (DAOPHOT parameters $\chi$ and sharp), and $x y$-pixel coordinates were determined for each object by the stand-alone task ALLSTAR using the best-fit PSF (determined from $\gtrsim 20$ stars). Frames from a single pointing were matched using the task DAOMASTER. The now single set of $x y$-pixel coordinates were converted to celestial coordinates (J2000.0) using a reference list of stars from the USNO-A2.0 (Monet et al. 1998) catalog imported into the IRAF task tfinder.

The Washington+DDO51 standard fields SA98, SA110, SA114, and NGC3680 (Geisler 1990, 1996) were observed interspersed with object frames during photometric nights. Aper-

\footnotetext{
${ }^{2}$ IRAF is distributed by the National Optical Astronomy Observatories, which are operated by the Association of Universities for Research in Astronomy, Inc., under cooperative agreement with the National Science Foundation.
} 
ture corrections were obtained using the program DAOGROW (Stetson 1990). Photometric transformations including airmass, color (if necessary), and nightly zero-point terms were determined as described in Majewski et al. (1994), utilizing the matrix inversion algorithm of Harris, Fitzgerald \& Reed (1981) incorporated into a local code (see also discussion in Paper IV). Five of the six observing runs had at least one night for which photometric transformation coefficients could be derived.

All observed pointings were locked onto a single magnitude system in the following way. For photometric frames, the measured magnitude of each individual star was calibrated using the nightly photometric transformation coefficients as derived from our standard stars. Stars observed in multiple photometric frames were used to determine residual relative magnitude zero-point offsets between these frames. Zero point corrections were applied to correct the photometric frames to a mean level between frames, and these corrections were applied iteratively until the zero-point shifts were all less than 0.001 magnitudes. For the photometric frames, initial offsets were $<0.04$ magnitudes in all frames and $<0.01$ in $87 \%, 83 \%$, and $74 \%$ of all $M, T_{2}$, and DDO51 frames, respectively. In each subsequent bootstrapping step, multiple non-photometric fields were matched to the existing calibrated database, overlapping stars were used to determine frame-by-frame zero-point offsets and color terms, and the derived terms were used to convert the non-photometric magnitudes to the calibrated system using an algorithm similar to that used for the photometric transformation coefficients. All 96 fields were matched and calibrated for a final time using the frame-by-frame photometric or bootstrapped transformation equations. Again, zero-point shifts were iteratively applied until all shifts were less than 0.001 magnitudes. For the entire database, initial zero-point offsets were always $<0.04$ and $<0.01$ magnitudes in $88 \%, 87 \%$, and $82 \%$ of all $M, T_{2}$, and DDO51 frames, respectively.

Reddening values were found toward each individual star in our survey using maps from Schlegel, Finkbeiner \& Davis (1998). These reddening values have a mean of $E(B-V)=$ 0.0204 and a standard deviation of 0.0044. Extinction and reddening values in our observed $M, T_{2}$, and $D D O 51$ bands were calculated according to equations from $\S 2.2$ of Paper I. Henceforth, all magnitudes and colors given are extinction and reddening corrected and we omit the "dereddened" subscript from our magnitudes and colors for brevity (e.g., $\left.M_{0} \equiv M\right)$.

Figure 2 gives the photometric errors calculated for all photometered objects in each filter in the survey area (Figure 1). Any star having measurement errors larger than $\epsilon_{M}=$ $0.11, \epsilon_{T_{2}}=0.13$, and $\epsilon_{D D O 51}=0.10$ is removed from our search for Sculptor stars. (See $\S 4.6$ for a discussion of contamination of our ultimate sample due to photometric uncertainties.) Through an analysis of the PSF-fitting parameters $\chi$ and sharp, the sample is further limited to those objects with stellar morphologies. The stellar locus of $\chi$ and sharp values for 
each CCD frame was shifted to bring them to common values of 1 and 0 , respectively, so that a single set of limits $(\chi<1.18$ and $-0.25<$ sharp $<0.25)$ could be invoked across the dataset to select acceptable stellar objects as shown in Figure 3. These morphological limits eliminate most sample contamination by galaxies (large $\chi$ at intermediate and faint magnitudes), poorly imaged stars (large \pm sharp), and saturated stars (large $\chi$ at bright magnitudes).

The varying conditions under which the survey was taken results in a varying sensitivity across the survey (evident in the apparently non-smoothly varying density of detected sources in Figure 1), and requires us to determine the limiting magnitude of each field so that homogeneous samples can be created for structural analysis of Sculptor (§5). Magnitude limits for each frame were set to the mean magnitude value at the imposed error limits of the data. A histogram of the number of pointings per magnitude limit is shown in Figure 4.

\section{SELECTION OF SCULPTOR MEMBER STAR SAMPLES}

This section describes the creation of magnitude limited samples of likely Sculptor giant star members. Individual stars are subject to the following criteria: First, the stars must have magnesium line/band strengths consistent with those for metal-poor giant stars as gauged in the $\left(M-T_{2}, M-D D O 51\right)$ diagram, hereafter referred to as the two-color diagram (2CD). Second, those stars selected as giant stars must have combinations of effective temperatures and apparent magnitudes consistent with the RGB and/or red horizontal branch (RHB) of Sculptor in the $\left(M-T_{2}, M\right)$ color-magnitude diagram (CMD). Ideally, a third criterion would be that the stars must have heliocentric radial velocities, $v_{\text {hel }}$, consistent with the systemic radial velocity of the Sculptor dSph. However, we have to date spectroscopically observed only a small sample $(\sim 10 \%)$ of our selected Sculptor giant candidates. Nevertheless, those spectroscopic data that we have obtained verify the robustness of the other selection criteria toward selecting bona fide Sculptor giant stars (§4.5).

A second sample of stars useful for exploration of the Sculptor morphology are the BHB stars. The near-complete isolation of this very blue population from the bulk of foreground MW contaminants allows for a qualitative check on the distribution the more numerous and brighter red giant stars. Stars satisfying either the RGB/RHB or BHB criteria are then broken into various magnitude-limited samples that give different sampling areas and sampling densities of the Sculptor field. 


\subsection{The Two-Color Diagram}

The $\left(M-T_{2}, M-D D O 51\right) 2 \mathrm{CD}$ for stars in the area surveyed around the Sculptor $\mathrm{dSph}$ is shown in Figure 5. The separation of giant and dwarf stars in the 2CD is based on the DDO51 filter measure of the $\mathrm{Mg}$ I triplet at $5150 \AA$ and the $\mathrm{MgH}$ feature with bandhead at $5211 \AA$ (Paper I). At a given stellar surface temperature, these spectral features are primarily sensitive to stellar surface gravity and secondarily to metallicity. The $M$ broadband filter is used as a measure of the continuum flux across the wavelength range of the magnesium features so that the $(M-D D O 51)$ color index yields an effective measure of the stellar surface gravity. For K spectral type stars, giants have larger ( $M-D D O 51)$ due to weaker magnesium features. The giant/dwarf star separation becomes less pronounced and disappears at both earlier and later spectral types. To sort stars by effective temperature, we use the $T_{2}$ broadband filter to create the color index $\left(M-T_{2}\right)$, which primarily reflects stellar effective temperatures (Paper I; Bessell 2001).

The secondary dependence of the magnesium features on metallicity also allows for formulation of isometallicity loci for both dwarfs and giants (Paper I; Paltoglou \& Bell 1994), as shown in Figure 5. Thus, in principle we can tune our selection to giant stars of a given metallicity. The isometallicity loci (as displayed in Figure $2 \mathrm{~b}$ of Paper I) are given for both dwarfs and giants; increasing metallicity correlates with a decrease in $(M-D D O 51)$ index. As in Paper I, a shift of -0.005 was applied in $(M-D D O 51)$ and -0.075 in $\left(M-T_{2}\right)$ from the curves of Paltoglou \& Bell (1994); this shift, which causes better coincidence of the Paltoglou \& Bell (1994) solar metallicity dwarf curve with our data from this and prior studies, can be understood in light of the fact that the Paltoglou \& Bell (1994) passbands were nonstandard (as discussed in Lejeune \& Buser 1996, and Paper I). These isometallicity loci are used to shape a $2 \mathrm{CD}$ giant selection region (Figure 5) that excludes dwarfs with $[\mathrm{Fe} / \mathrm{H}]>-2.0$ while selecting the dominant populations of Sculptor giants, which have metallicities of $[\mathrm{Fe} / \mathrm{H}] \sim-1.5$ and $[\mathrm{Fe} / \mathrm{H}] \sim-2.3$ (Kaluzny et al. 1995; Majewski et al. 1999). The allowable temperature range for stars selected to be Sculptor giant candidates with this initial criterion is set to span from the blue edge of the MW field population redward to just beyond the color of the tip of the Sculptor RGB. Both diagonal edges of the selection region are set approximately parallel to the dwarf locus but offset to redder color. This offset will help limit the number of dwarfs accidentally landing within the selection region due to photometric errors.

Stars enclosed in the adopted 2CD giant region are only giant star candidates, and, moreover, they are giant candidates not necessarily specific to the Sculptor dSph. Other objects that may fall within this selection region could include (1) field dwarfs that are scattered into the giant region by photometric error, (2) compact galaxies (typically dominated 
by giant star light and possibly at redshifts where the Mg features shift out of the DDO51 passband) that were not eliminated by the morphological limits, (3) field giants, and (4) metal-poor subdwarfs with $[\mathrm{Fe} / \mathrm{H}] \lesssim-2.0$. An analysis of the level of contamination that we expect is presented in $\S 4.5$.

\subsection{The Color-Magnitude Diagram}

The $\left(M-T_{2}, M\right)$ CMD, is presented in Figure 6. The RGB and BHB sequences of Sculptor clearly stand out. Below, we describe in detail how Figures 5 and 6 together are used to isolate these populations from the Galactic foreground contamination.

\subsubsection{Selection of Candidate Sculptor Stars from the Giant Star Sample}

Figure $6 \mathrm{~b}$ shows that the Sculptor RGB is a predominant feature of the stars passing the $2 \mathrm{CD}$ giant star selection criteria. Application of a second criterion defined in the CMD will further purify the Sculptor giant sample. Limit definitions for this selection are guided by two methods illustrated in Figure 7. First, based on stellar proper motions found by Schweitzer et al. (1995), those giant stars with a high probability (> 80\%) of being Sculptor member stars are used to define the RGB locus to a magnitude of $M \sim 20.5$ (Figure 7a); third order polynomials have been defined to enclose this astrometric sample. A second third order polynomial is used to retain a small region of the CMD centered at $\left(M-T_{2}, M\right) \approx(0.97,19.9)$, where there is a concentration of highly probable Sculptor giants.

Although the high probability proper motion members provide a useful means to constrain the RGB for $M \lesssim 20.5$, much of our survey extends fainter than this. Unfortunately, near the higher density dSph center where the RGB is most clearly defined, our data are actually shallower than in some of the more outlying fields of our survey. However, deeper $B$ and $V$ Sculptor photometry from (Majewski et al. 1999) can be used as a substitute. The Majewski et al. data (1) show a separation of the RHB and the RGB that is actually more pronounced in $(B-V, V)$ space than in $\left(M-T_{2}, M\right)$ space, and (2) extend to magnitudes required to define RGB bounding limits suitable for our deepest $M, T_{2}$ data (see Figure 1 of Majewski et al. 1999). We isolate the RHB using

$$
\begin{gathered}
0.4<(B-V)<0.64 \\
-0.38(B-V)+20.35<V<-0.38(B-V)+20.6
\end{gathered}
$$


and isolate the lower RGB by selecting

$$
\begin{gathered}
(B-V)<0.85 \\
V>20.05 \\
V>-6.67(B-V)+24.38 .
\end{gathered}
$$

All stellar objects that have photometry from both this study and Majewski et al. (1999) are shown in Figure 7b, regardless of magnitude errors in either. Guided by those stars satisfying Equations 1 and 2, we extend the RGB/RHB selection to $\left(M-T_{2}\right)=0.65$ between $20.0<M<20.47$ to include RHB stars and find it reasonable to simply extend our third order polynomials to $M=21.0$ for the RGB fainter than $M=20.5$. The final adopted CMD selection criterion is applied to all stars previously selected as giant star candidates in the 2CD over our entire survey area (Figure 7c).

Note that in the RHB selection we are not attempting to include all RHB stars in our survey. Rather, we are only attempting to include as many probable Sculptor stars from our giant candidate sample as possible, after delineating that population by appealing to a data set where the RHB is more distinct from the RGB.

\subsubsection{Selection of Candidate Sculptor BHB Stars}

The extreme blue colors of BHB stars relative to the MSTO of the MW field population provides a unique opportunity to isolate a fairly pure sample of Sculptor BHB candidates using only a CMD selection. However, as seen by comparing the BHB in Figures 6a and $6 \mathrm{~b}$, imposition of the previously adopted magnitude error limits substantially reduces the number of selected stars from the very blue end of the BHB, primarily due to limitations of the $T_{2}$ photometry. However, because the BHB is so blue, for this CMD selection we can

tolerate larger $\left(M-T_{2}\right)$ color errors (along the BHB) with little decrease in sample purity (see Table 6 for the mean color error of this sample). Therefore we do not limit the selection of BHB stars by their photometric error. The Sculptor BHB candidate selection procedure is demonstrated in Figure 8.

As with the RGB/RHB selection, we again limit the stars used for definition of the bounding limits to those having Sculptor proper motion membership probabilities greater than $80 \%$ (Figure 8a). In the definition of the BHB selection, the three main considerations are: (1) enclosure of the available blue extent of the BHB stars, (2) inclusion of RR Lyrae stars (which, due to variability, will have a larger magnitude scatter than non-variable HB stars), and (3) minimization of error-scattered contaminants from the "blue edge" of the MW field dwarf population. The final selection criteria are applied to the entire survey as 
demonstrated in Figure 8b. For stars with $\left(M-T_{2}\right)>0.6$, the approximate blue edge of the MW field population, error bars in both color and magnitude are shown to demonstrate the low probability that a star from this population might be scattered into the BHB selection region.

\subsection{Definition of Magnitude Limited Samples}

Figure 9 shows the final CMD and spatial distribution of objects selected from our survey to be candidate Sculptor members and those objects pruned from further analyses. Color and magnitude error bars are included on individual candidate points in Figure 9a to give a sense of the security of the sample definitions with respect to their photometric errors. We also include average color and magnitude errors in 0.25 magnitude bins for the selected Sculptor candidate sample and for the de-selected, "field star" sample. These average errors demonstrate that the quality of data between the field and candidate lists are comparable, but, in detail, the data outside the core of the galaxy are demonstrably of greater average quality than data taken near the Sculptor core. From Figure 9, we note: (1) the spatial extent of our selected BHB stars roughly coincides with that of our selected RGB/RHB stars, (2) there seem to be a significant number of stars that satisfy the RGB/RHB or BHB selection criteria yet fall outside the King limiting radius as determined by IH95 (these constitute a "break population" in the radial profile, as shown below), (3) the distribution of some of our selected stars fall along field boundaries due to the increased magnitude depth in overlap regions, (4) there does not seem to be an inordinate excess of stars included in our selected sample based on the fact that there does not seem to be a deficit of stars within the RGB boundary of the pruned star CMD in Figure 9c, but, on the contrary, (5) our selection is conservative in order to be reliable at the expense of being complete. That we are missing some Sculptor giant/HB stars is most obvious by the greater density of stars at the base of the RGB in Figure 9c, and by a residual concentration of stars near the spatial center in the deselected sample shown in Figure 9d. Sculptor stars may be omitted by the joint 2CD and CMD selection criteria due to: (1) the decreasing sensitivity of the 2CD to the giant/dwarf discrimination on the lower RGB and sub-giant branch, (2) RHB stars that fall outside the 2CD and/or CMD selections, (3) asymptotic giant branch or RGB stars that fall outside the RGB/RHB selection at bright magnitudes, (4) RR Lyrae stars in variability phases that place them outside our "BHB" selection, and (5) stars with photometric errors that scatter the data points beyond our selection limits.

Sculptor candidates thus far selected by application of the 2CD and CMD criteria provide a useful database for follow-up spectroscopy and exploration of Sculptor dynamics; 
however, a study of the morphological structure of Sculptor requires homogeneously selected samples that account for variable magnitude limits across the survey area. To do this, we analyze the spatial distributions of various subsamples of Sculptor candidates after imposing specific magnitude limits (those adopted for the RGB/RHB samples are shown as dashed lines in Figure 9a) and eliminating survey regions with imaging depths shallower than these limits from consideration. For the RGB/RHB sample, the various adopted magnitude limits trade off the balance between sky coverage and depth. An $M \leq 19.0$ limit reaches the vast majority of our fields (Figure 4) and it likely gives us the purest sample of Sculptor RGB stars, albeit at the expense of a faint survey depth. A second, $M \leq 20.3$ limit gives the maximum depth that includes most of the CCD fields near the center of Sculptor. A final, $M \leq 21.0$ limit plumbs the deepest fields in our survey, but over rather limited areas. The latter sample is put to limited use because it does not include the central fields and is partially limited by poorer $T_{2}$ magnitudes. As discussed in $\S 3.2 .2$, the magnitude errors allowable for the BHB sample have been loosened in order to reclaim a larger number of BHB stars. Magnitude limits that are adjusted for this different error sample (not shown in Figure 4) are used to define sample magnitude limits of $T_{2} \leq 19.9$ and $T_{2} \leq 20.3$ for our BHB samples (see Figure 9a). Also, any field that did not have a corresponding $M$ depth that allowed sampling of the full BHB color range (i.e. requiring $19.5 \leq M \leq 20.6$ to match the two respective BHB $T_{2}$ limits adopted above) is automatically rejected. In the following discussions, the above defined subsamples will be referred to by their imposed magnitude limits (i.e. the RGB/RHB limited to $M \leq 19.0$ is the $M \leq 19.0$ sample and the BHB limited to $T_{2} \leq 19.9$ is the $T_{2} \leq 19.9$ sample, etc.). The spatial distribution and field limits of each sample are shown in Figure 10.

\section{SPECTROSCOPY}

The reliability of our photometrically selected Sculptor candidates (RGB/RHB and BHB) can be assessed via radial velocity membership checks. A complete census would require $\sim 2700$ spectra; limited allotted observing time and poor conditions during that allotted time has precluded us from fulfilling this task. However, we have obtained spectra for $\sim 5 \%$ of our photometrically selected Sculptor candidates (mainly for $\sim 10 \%$ of our RGB/RHB sample) and this allows us to at least gauge the statistical reliability of our selection methodology in the magnitude range probed by the obtained spectroscopy. This section describes our spectroscopic observations and an assessment of the contamination of our candidate sample. 


\subsection{CTIO HYDRA Spectroscopy}

Spectra for this study were obtained using the HYDRA multi-fiber system at the Blanco 4-m telescope at the Cerro Tololo Inter-American Observatory (CTIO) during UT 10 12 November 2000 and UT $7-10$ October $2001^{3}$. The Loral $3 \mathrm{~K} \times 1 \mathrm{~K}$ CCD and the 790 lines/mm KPGLD grating (in first order) were used for observations during November 2000 and yield a resolution of $\sim 2600$ (or $2.6 \AA$ per resolution element) with spectral coverage from

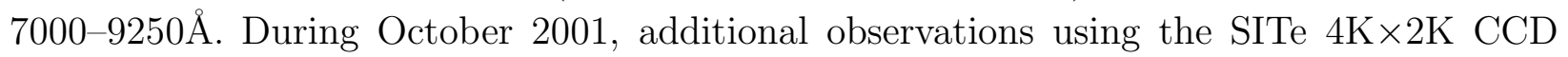
and the 380 lines/mm grating (in first order) were made. A $200 \mu \mathrm{m}$ slit plate was placed after the fibers to improve the resolution to $\sim 7600(1.2 \AA$ per resolution element) with a spectral coverage from 7750-8700A. During both runs, calibration lamp (Penray HeNeArXe) exposures were taken in every HYDRA fiber setup for wavelength calibration. Each standard radial velocity (RV) calibrator was observed through nearly a dozen individual fibers in each HYDRA setup; 6 - 13 of these calibrators were observed during each run.

As with the imaging frames, routines from the IRAF imred.ccdred package were used to reduce the raw CCD multi-fiber data. Images were bias subtracted, overscan corrected, trimmed, and then corrected for pixel-to-pixel sensitivity variations and chip cosmetics by applying "milky flats" as described in the CTIO Hydra manual by N. Suntzeff ${ }^{4}$. Subsequent extraction and calibration of the individual fiber spectra were completed with a local IRAF code incorporating functions from the imred.hydra package as described in Paper VI.

The RV of each observed star is determined via a modified version of the cross-correlation (XC) technique developed by Tonry \& Davis (1979). Spectra are Fourier-filtered to remove low-order (continuum) variations and then cross-correlated against a master RV template spectrum. The master is a normalized spectrum that has all spectral regions that contribute little more than noise to the XC function masked (i.e. set to one). Masking of the spectra eliminates all but the most vertical parts of the uncontaminated spectral lines, which contains the most information for the XC function. The foundations and application of this XC methodology are described more fully in Majewski et al. (2004a) and Paper VI.

Each derived RV is assigned a numerical quality index (with $Q=7$ being the highest quality, $Q=1$ the lowest; see Majewski et al. 2004a) gauged by the strength (as compared to secondary peaks) and symmetry of the XC peak. It is found that RVs with $Q>3$ yield

\footnotetext{
${ }^{3}$ These allocations were also used for observations of Carina dSph stars presented in Paper VI where a more lengthy discussion of the observations and analyses for these data are given. We only summarize this previous presentation here; readers can see Paper VI for more detail.

${ }^{4}$ http://www.ctio.noao.edu/spectrographs/hydra/hydra-nickmanual.html
} 
measurements that are acceptable for membership discrimination, but the RV errors improve with higher $Q$. We set $Q=8$ for stars that have average velocities from multiple observations $^{5}$. The dozens of single observations of RV standards show typical standard deviations of $5 \mathrm{~km} \mathrm{~s}^{-1}$ for the November 2000 data and $2 \mathrm{~km} \mathrm{~s}^{-1}$ for the higher resolution October 2001 data. We adopt typical errors that are twice the standard deviations determined above for our Sculptor target stars due to the lower $S / N$ of these observations compared to the RV standard observations. These adopted values are consistent with comparisons shown in Table 2 (see below for the Table description) for multiply observed stars and reasonable for the higher quality $(Q>5)$ spectra. Although five different HYDRA setups were used for Sculptor observations, mediocre to poor observing conditions meant that not all targeted stars yielded usable spectra; therefore, the number of useful spectra are significantly less than the maximum possible with Hydra's 64 or 128 available fibers on these observing runs, despite exposure times of up to 4.5 hours. On the other hand, the number of Hydra fibers actually exceeds the density of Sculptor targets, so that some unassigned fibers could be placed on stars other than those in our selection sample at no cost. Thus we were able to target some additional (brighter) stars with photometric properties placing them outside our selection criteria; these stars are useful to get some idea of the incompleteness of our Sculptor candidate sample.

\subsection{Magellan MIKE Spectroscopy}

Spectra were also obtained with the Magellan Inamori Kyocera Echelle (MIKE) slit spectrograph (Bernstein et al. 2003) mounted on the Magellan II (Clay) 6.5-m telescope at the Las Campanas Observatory on the nights of UT 27 - 28 January 2004 and 30 31 December 2004. Reduction of the data from raw detector output to stellar $v_{\text {hel }}$ used fundamentally the same tools as the CTIO HYDRA reductions described above, with the addition of one step to account for slit centering errors (described below). A more extensive discussion of the reduction of these MIKE data is given (in the context of the Carina dSph stars observed on the same observing run) in Mu05b. For XCs to obtain $v_{\text {hel }}$, we used a single red order (at $R=19000$ ) of the double echelle spectrograph. This order covers the region $8468-8693 \AA$ and includes the strong Ca II infrared triplet as well as just over a dozen other lines that contribute usefully to the XC. Two other orders were cross-correlated separately

\footnotetext{
${ }^{5}$ These stars are not necessarily of higher quality than some individual $Q=7$ observations; the $Q=8$ value just serves to distinguish an RV that is averaged over multiple measures from an RV from a single spectrum. However, in all but one case at least one $Q=7$ measure contributes to the weighted averaged value
} 
but these did not improve the precision of the derived $v_{\text {hel }}$, even when all three of the $\mathrm{XC}$ functions were combined. RV standards were observed both at the beginning and ending of the night while a ThAr calibration lamp was observed on average once per hour throughout the night (though the instrument proved extremely stable over the night). The RV standards are used to measure small induced offsets in the derived radial velocities that are particular to the adopted radial velocity XC template. Bias frames, exposures of the quartz dome lamp, and "milky flats" were observed on a nightly basis to correct for large-scale as well as pixel-to-pixel throughput variations. Because all of the stars were observed through the $0^{\prime \prime} .9$ slit, whereas the typical seeing was about 0.7 , significant fractional errors in derived RVs may arise from slit centering errors. To measure the velocity shifts that result from this effect, we independently cross-correlate the telluric absorption features in each order for each star against those in a set of observed radial velocity standards as well as in dusk spectra (see discussion in Paper X).

Given the resolution and average quality of data, the MIKE observations should have a velocity precision of $\lesssim 1 \mathrm{~km} \mathrm{~s}^{-1}$. In practice, however, the derived $v_{\text {hel }}$ have a precision of closer to $1.5 \mathrm{~km} \mathrm{~s}^{-1}$, the degradation arising primarily due to the precision of the literature-

quoted RV values of the standards. The velocity precision is also somewhat limited by the slit illumination errors, which, in fact, vary with wavelength due to atmospheric dispersion. This atmospheric dispersion introduces a wavelength dependent term in the slit illumination that is hard to characterize without multiple observations with MIKE. The errors for spectra taken in January 2004, $\pm 1.5 \mathrm{~km} \mathrm{~s}^{-1}$, are slightly lower than the $\pm 2.5 \mathrm{~km} \mathrm{~s}^{-1}$ assessed for the December 2004 data due to difficulties in the reduction of the latter. These errors are more than suitable for evaluation of kinematic membership and acceptable for assessing velocity dispersion, $\sigma_{v}$, measurements for Sculptor.

\subsection{Radial Velocity Summary}

In total, 194 high- to mid-quality spectra were obtained in the three runs (49 in 2000, 111 in 2001, 22 in January 2004, and 12 in December 2004), of which 157 were of unique stars. Based on our photometric selection, 11 of these stars were expected to be field stars, two were expected to be Sculptor BHB stars, and the rest were expected to be Sculptor RGB/RHB stars. As in Paper VI, for stars observed multiple times we calculate weighted averages, $\left\langle v_{\text {hel }}\right\rangle$, of the individual measured velocity values, $v_{i}$, following

$$
\left\langle v_{\text {hel }}\right\rangle=\sum_{i}\left(\omega_{i}^{2} v_{i}\right) / \sum_{i}\left(\omega_{i}^{2}\right)
$$


Weights used in Equation 3 are defined by

$$
\omega_{i}=\omega_{\text {quality }, i} \times\left(1 / \epsilon_{v_{i}}\right)
$$

and account for varying $S / N$ and resolution in the spectral observations. As discussed in the previous sections, we assume $\epsilon_{v_{i}}=10 \mathrm{~km} \mathrm{~s}^{-1}, \epsilon_{v_{i}}=4 \mathrm{~km} \mathrm{~s}^{-1}, \epsilon_{v_{i}}=1.5 \mathrm{~km} \mathrm{~s}^{-1}$, and $\epsilon_{v_{i}}=2.5 \mathrm{~km} \mathrm{~s}^{-1}$ for representative relative velocity errors for the November 2000, October 2001, January 2004, and December 2004 observations, respectively. The previous use of our $\mathrm{XC}$ methodology has shown the magnitude of the $\mathrm{XC}$ peak $(C C P)$ to be correlated with the velocity precision (i.e. a stronger CCP yields a better velocity measurement); thus we include a "quality" term, $\omega_{\text {quality }, i}$, in weighting each RV. Measurements of $C C P$ in the ranges $(<0.3) / 0.3-0.5 / 0.5-1.0 /(\geq 1.0)$ are assigned $\omega_{\text {quality }, i}=0.5 / 1.0 / 2.0 / 3.0$, respectively (Paper VI).

The individual observations and adopted mean velocities for the 36 stars with multiple observations are listed in Table 2 . The first line of each entry gives the calculated $\left\langle v_{\text {hel }}\right\rangle$ and standard deviation, $s\left(v_{\text {hel }}\right)$, of the individual measures of $v_{\text {hel }}$. The Table also serves to show that for stars observed twice in the same run, the adopted uncertainties are generally consistent with $s\left(v_{\text {hel }}\right) / \sqrt{N}$ ( $N$ is the number of individual measures of the velocity). However, we recognize the low numbers in the statistics of this agreement. For the stars 7015937, 31000262 , and 31000811, $s\left(v_{\text {hel }}\right)$ is significantly different from the adopted $\epsilon_{v_{i}}$ due to largely disparate velocities between observations. This is almost certainly due to an incorrect determination of $v_{\text {hel }}$ and/or $Q$ for at least one of the spectra of these stars, not an intrinsic change in the velocity of the observed star (and we note that one or more of the RVs is near the systemic RV of Sculptor in each case). We reobserved 31000262 and 31000811 in December 2004 with the MIKE echelle to clarify these as members/non-members. The star 31000262 is confirmed as a member while 31000811 is not; however, their $s\left(v_{\text {hel }}\right)$ are still large. For four other stars $(1000844,1008239,28000278$, and 28000376) the difference in velocities are larger than $\sqrt{N} \times$ the adopted error estimates; though binary stars may play a role here, with a normal distribution of errors we would of course expect greater than 1- $\sigma$ differences to occur one third of the time - or for about 12 stars in Table 2. That we obtain half this number overall likely reflects that we have conservatively overestimated the true RV uncertainties. Nonetheless, these seven stars with large internal RV differences are omitted from measurement of the Sculptor systemic velocity, $v_{\text {sys }}$, and $\sigma_{v}$.

Along with internal consistency, we also check our derived $v_{\text {hel }}$ against 10 stars with previous RV measurements (Armandroff \& Da Costa 1986; Queloz, Dubath \& Pasquini 1995, hereafter $\mathrm{QDP})^{6}$. The comparison between the literature values for these stars and those

\footnotetext{
${ }^{6} \mathrm{~A}$ larger number of overlap stars may be checked against the Tolstoy et al. (2004) database but tabulated
} 
obtained for the current study are listed in Table 3. As stated earlier, stars with weighted velocity averages from our own study have been given $Q=8$ to distinguish them from single observations. The Table shows general consistency between previous observations and those velocities determined for this study. More specifically, our observations more closely match those from the more recent QDP study.

Finally, in Table 4 we present all of the available radial velocities for the Sculptor dSph. For the sake of internal consistency, our determined $v_{\text {hel }}$ are used instead of literature observations when both are available. The 22 literature stars that were not reobserved for this study are also given. When both literature sources had observations, the error-weighted mean velocity and its associated error are given ${ }^{7}$. Along with RV statistics, the Table also includes color-color-magnitude measures and the elliptical distance for each star assuming the mean ellipticity for the samples fit in §5.2. Finally, the last column allows for gauging the accuracy of our photometric selection technique (see below). Those stars satisfying the defined criteria are denoted with a "Y" while those that do not are denoted with a "N". So, for example, a star that satisfies the photometric criteria $(\mathrm{P})$ but not the radial velocity criterion (V; defined below) has a "P/V" entry of "Y/N". Several special cases are highlighted by footnotes to the Table.

Figure 11 gives the distribution of the spectroscopically observed stars from Table 4 both in (semi-major axis) distance-velocity space and on-sky. From Figure 11a, it is immediately apparent that the vast majority of the observed stars are kinematically similar. Given the observations are nearly in the direction of the South Galactic Pole (SGP), typical MW field dwarfs (mainly from the disk at these magnitudes) and even the bulk of halo field giant contaminants will be at lower heliocentric velocity than Sculptor so that the dSph members should be fairly distinct. For a population of stars with net zero revolutionary velocity about the Galactic center viewed in the direction of Sculptor, the mean heliocentric velocity will be about $35 \mathrm{~km} \mathrm{~s}^{-1}$; for higher, net positive revolutionary speeds, the mean heliocentric velocity decreases towards $0 \mathrm{~km} \mathrm{~s}^{-1}$. To determine the velocity of Sculptor, the mean of all available velocities was calculated using a $3 \sigma$ rejection iterated until points were no longer rejected

data are not readily available.

${ }^{7}$ We note that QDP argue that their observations of H185 and H512 are significantly different from Armandroff \& Da Costa (1986). Star H512 was not observed by us. Both of our observations (see Table 2) for H185 (our 1002429) are consistent with that found by QDP but not Armandroff \& Da Costa (1986). If a binary, it is possible that we happened to observe H185 at the same phase as QDP. Nevertheless, according to our spectroscopic membership criterion given below, all observations of both stars are consistent with them being Sculptor members, but, for consistency with QDP, H185 is omitted from our systemic velocity and velocity dispersion measurements (even though doing so has an insignificant effect on the results). 
to obtain $\overline{v_{\text {hel }}}=107.96 \pm 0.76 \mathrm{~km} \mathrm{~s}^{-1}$, in good agreement with the previously determined Sculptor $v_{\text {sys }}\left(\mathrm{QDP}: v_{\text {sys }}=109.9 \pm 1.4 \mathrm{~km} \mathrm{~s}^{-1}\right)$. The $3 \sigma$ rejection algorithm essentially sets the RV membership criterion to $80 \leq v_{\text {hel }} \leq 135$, very similar to the limits set by Tolstoy et al. (2004) for their Sculptor member search. Stars falling within this velocity range we henceforth consider to be kinematically associated with the Sculptor dSph. A histogram of the distribution of stars with observed RVs is shown in Figure 11b and appears to follow a normal distribution centered on the determined mean velocity. Of particular interest in this set are the probable member stars outside our derived King limiting radius (\$5.2), one of which is a selected BHB star.

\subsection{Preliminary Dynamical Information from the Radial Velocity Survey}

Using the 134 stars leftover after pruning (1) stars with large $s$ in Table 4 (e.g., possible binaries, including H185, or problem RV measures), and (2) observations from November 2000 (due to the large velocity uncertainties for these spectra), Figure 12 gives the azimuthally averaged $v_{\text {hel }}$ and $\sigma_{v}$ profiles. The mean velocity $\left(\left\langle v_{\text {hel }}\right\rangle\right)$, velocity error $\left(\epsilon_{\left\langle v_{\text {hel }}\right\rangle}\right)$, and $\sigma_{v}$ are derived by the same formulae given by Mateo et al. (1991). The weighting scheme for calculation of $\left\langle v_{\text {hel }}\right\rangle$ here $\left(w_{i}=1 / \epsilon_{v_{i}}^{2}\right.$ with $\omega_{i}^{2}=w_{i}$ in Equation 3$)$ assumes the adopted, run specific $\epsilon_{v_{i}}$ values given above for the individual spectra. For the multiply observed stars in Table 2, the error as calculated for an error weighted mean $\left(\epsilon_{v_{\text {hel }}}=1 / \sqrt{\sum\left(w_{i}\right)}\right)$ is used; these values are tabulated in Table 4 . The error in the velocity dispersion, $\epsilon_{\sigma_{v}}$, is obtained from

$$
\epsilon_{\sigma_{v}}^{2}=\frac{\sigma_{v}^{4}+\left\langle\epsilon_{v_{i}}\right\rangle^{4}}{2 N} \frac{1}{\sigma_{v}^{2}-\left\langle\epsilon_{v_{i}}\right\rangle^{2}},
$$

where $\left\langle\epsilon_{i}\right\rangle$ is the average error in the velocity measurement and $N$ is the number of $\operatorname{stars}^{8}$. Equation 5 results in an indeterminable $\epsilon_{\sigma_{v}}$ when $\sigma_{v}^{2} \leq\left\langle\epsilon_{i}\right\rangle^{2}$; an indeterminable $\sigma_{v}$ results when $N \epsilon_{\left\langle v_{\text {hel }}\right\rangle}^{2} \leq\left\langle\epsilon_{i}\right\rangle^{2}+\epsilon_{\left\langle v_{\text {hel }}\right\rangle}^{2}$. Only bins that contain nonzero, determinable $\sigma_{v}$ and $\epsilon_{\sigma_{v}}^{2}$ are shown in Figure 12. To demonstrate possible biases caused by binning, the results for the data are supplied for three different bin sizes.

The velocity profile of Sculptor is, in general, constant with semi-major axis distance. We have confirmed that this is the case for velocity as a function of either equatorial coordinate as well. The velocity profile is no better fit with a linear relationship between $v_{\text {hel }}$ and $\alpha, \delta$, or $a$ than it is with a constant value regardless of the bin size. Therefore, the data sug-

\footnotetext{
${ }^{8}$ Note that $\sigma_{v}, \epsilon_{\left\langle v_{\text {hel }}\right\rangle}$, and $\left\langle\epsilon_{v_{i}}\right\rangle$ given here are, respectively, $\mu_{i n t}, \sigma_{\langle v\rangle}$, and $\left\langle\sigma_{i}\right\rangle$ in the nomenclature of Mateo et al. (1991).
} 
gest Sculptor is not rotating at least at the level of the observed dispersion in the velocities $\left(\sqrt{N} \epsilon_{\left\langle v_{\text {hel }}\right\rangle} \sim 9 \mathrm{~km} \mathrm{~s}^{-1}\right)$. However, we note that our RV sampling of Sculptor candidate members becomes poor beyond $a \sim 30^{\prime}$; velocity trends beyond this radius are not well constrained by our present data. Using the trimmed sample of stars presented in Figure 12 and the above error weighting scheme, the mean velocity of Sculptor is $\left\langle v_{\text {hel }}\right\rangle=110.43 \pm 0.79 \mathrm{~km} \mathrm{~s}^{-1}$.

We find a global dispersion of $\sigma_{v}=8.8 \pm 0.6 \mathrm{~km} \mathrm{~s}^{-1}$ for the 134 stars in Figure 12. While this is $40 \%$ higher than determined by QDP, these authors only probe out to $\sim 10^{\prime}$; as may be seen in Figure 12, our dispersion over this same radius is in fact comparable to that of QDP. On the other hand, our global $\sigma_{v}$ appears to be comparable to the global value given in the recent letter by Tolstoy et al. (2004), who actually survey a similar radial extent as we $\mathrm{do}^{9}$. This correlation of derived dispersions with radial coverage points to the likely existence of a radial gradient in Sculptor velocity dispersion, with a hotter dispersion found at larger radius. Such a trend is suggested by Figure 12, where, in general, colder dispersions are found at smaller radii, particularly when larger bin sizes are adopted to improve the errors on the dispersion. Clearly one would prefer better statistics at larger radii, but we note that a radial gradient is implied by the results of Tolstoy et al. (2004), who note that hotter dispersions attach to the more metal-poor Sculptor stars and that these stars have a larger radial extent than metal-rich Sculptor stars. Our own analysis of Sculptor shows a similarly distinct radial distribution of "metal-poor" and "metal-rich" populations (see discussion in $\S 5.4)$.

It seems difficult to escape the conclusion that the Sculptor dispersion profile is, at minimum, flat to a significant fraction of the King limiting radius, and could even be rising at large radius (at least there seems to be a rise in the dispersion past $\sim 0.4 r_{\text {lim }}$ ). This is a trend that is being found in other dSph systems (e.g., Mateo (1997); Kleyna et al. (2002, 2004); Mu05b). Predominantly bound stellar systems are generally expected to produce declining dispersion profiles because the Keplerian orbits of stars within the system should produce lower velocities at their apocenters. The implications of flat/rising profiles have been discussed by, e.g., Kroupa (1997) and Kleyna et al. (1999), who interpret such trends as an important signature of tidally disrupting systems. A flat/rising dispersion trend is also found in Ursa Minor to large radius (see Paper IX). Ironically, after measuring a similarly

\footnotetext{
${ }^{9}$ Tolstoy et al. (2004) derive different dispersions for Sculptor stars separated into metallicity groups, one with $[\mathrm{Fe} / \mathrm{H}]<-1.7$ and another having $[\mathrm{Fe} / \mathrm{H}]>-1.7$. The global $\sigma_{v}$ set by the present data is intermediary to those Tolstoy et al. report for these metallicity-separated populations. In $\S 5.4$ we make an approximately similar metallicity separation of Sculptor stars on the basis of photometry, however, this separation is not as reliable as the spectroscopic separation of Tolstoy et al.. We also have fewer overall Sculptor velocities than Tolstoy et al. with which to estimate reliably distinct dispersions by metallicity.
} 
flat profile in the Draco system, Kleyna et al. (2002) have appealed to an enormous dark matter halo, $(M / L)_{\text {tot }}=440 \pm 240$, as an alternative means to keep the dispersion hot to large radius. On the other hand, Mu05b show that prosaic models of tidally disrupting satellites with more modest mass and on very radial orbits can also give reasonable fits the similar $\sigma_{v}$ trend (including a centrally colder dispersion) and radial profile for the Carina $\mathrm{dSph}$. It is reasonable to expect similar models will work for Sculptor, which is at a similar Galactocentric radius and orbital phase as Carina. It is certainly compelling that the Sgr system also shows a flat/rising $\sigma_{v}$ trend Majewski et al. (2004a, 2005b). The structural and dynamical similarity of Sgr to other dSphs strongly begs the question of whether the disrupting Sgr is more dSph paradigm than exception (Majewski et al. 2005b). It is also worth noting that while the velocity distribution of stars in the inner parts of Sculptor is Gaussian, the distribution in the outer parts becomes fairly platykurtic — a distribution unexpected for a fully bound population of stars. These issues are explored further in Paper IX, Majewski et al. (2005b), and Mu05b. The main goal of this subsection is to demonstrate the apparent flat dispersion profile in Sculptor and point out the consistency of this trend with the interpretation that the Sculptor radial density break population we measure below (§5) may constitute unbound, tidal debris.

\subsection{Photometric Selection Accuracy}

A primary motivation for the RVs in the present study (including those with the lower velocity precision from observations in 2000) was to evaluate whether our photometric techniques to select Sculptor candidates are accurate so that we have confidence in the reliability of Sculptor structural properties derived from such data. Figure 11 summarizes the results of this program. The different symbols defined in the legend of Figure 11a reflect the various categories in column 10 of Table 4, where we correlate our photometric ( 33$)$ and RV (§4.3) membership criteria. Figure 13 displays the Sculptor field 2CD and CMD to illustrate the selection/de-selection of stars having derived RVs (where symbols are the same as in Figure 11). Figure 13 illustrates the efficacy of our photometric selection technique at isolating individual, highly probable Sculptor members; creating such reliable target lists is particularly helpful for ensuring cost-effective spectroscopic observations of the low-density regions of the dSph, where contamination by MW field stars becomes a more significant problem (see Figure 17 and $§ 5.2 .4$ ).

Table 5 summarizes the accuracy of the photometric selection criteria for the subsample

of spectra obtained to date. Given that most of the spectra obtained to date are for $M \lesssim 19$ candidates, the statistics are properly interpreted to represent our success for stars to these 
magnitudes, but do lend confidence that we can expect improved selection efficiency at fainter magnitudes over selection methodologies based on only CMD-position of candidates. The values given in the final column are the percentage of photometric selections that agree with the spectroscopic selection out of the total number of spectroscopically observed stars. Among all stars (BHB or RGB) we have selected to be photometric members that have RVs, a combined $94 \%$ are found to be spectroscopic members (i.e., only $6 \%$ are found to be false positives). Figure 11 shows evidence for a decrease in our false positive rate with radius from the center (i.e., with lower dSph density); beyond in $0.5 r_{\text {lim }}, 71 \%$ of our photometric members are confirmed spectroscopic members. This success rate can be compared to the $77 \%$ (308/401) velocity member success rate Tolstoy et al. (2004) found over their entire survey (which probes to similar magnitudes), a rate that declines to $\lesssim 45 \%$ for their stars observed beyond $0.5 r_{\text {lim. }}{ }^{10}$ Based on these trends, we predict that further study at even lower density regions of the $\mathrm{dSph}$ will show an even wider disparity in success rates between CMD-only and DDO51+CMD methods for picking candidate Sculptor stars.

Among our 22 photometrically-selected, non-members having derived RVs, nine have RVs consistent with Sculptor membership. That our photometric selection is missing some Sculptor members is consistent with our overall philosophy of sacrificing completeness for the sake of sample reliability. For purposes of mapping dSph structure, incompleteness removes signal whereas unreliable candidates add background. Our strategy is to aim for near-zero background to make sure diffuse components are mapped reliably, and this has driven adoption of rather conservative selection criteria. For example, four of the nine "false negatives" in Table 5 actually satisfy both the 2CD and CMD selection criteria in Figure 13; these Sculptor giants were lost from our sample because they failed our point source image shape criteria. Thus, these four stars (marked with footnote "f" in Table 4) are technically not failures of the Washington+DD051 giant selection method per se; nevertheless, we conservatively treat them as "selection failures" in our accounting. With the presently small spectroscopic sample of non-selected stars it is difficult to estimate our true sample incompleteness, especially given that it varies across the color-color-magnitude volume as a function of mainly stellar temperature and photometric error. Figure 13 in fact shows that the other five false negatives tend to lie just outside one of our selection limits, as might be expected. The resulting high degree of reliability of our photometric methodology is not unique to this study of Sculptor but is also true for our similar studies of Ursa Minor,

\footnotetext{
${ }^{10}$ To be fair, it is not clear from the Tolstoy et al. (2004) paper whether their results include some stars that were only observed because they had spare spectrograph fiber optic cables to place on stars that these authors would ordinarily consider to be poor candidates, though the bulk of their targets are clustered about the Sculptor RGB.
} 
Draco, Leo I, Andromeda I and Andromeda III (Paper IV; Paper IX; Paper X; Ostheimer et al. 2005).

\subsection{Review of Possible Sources of Contamination}

Our spectroscopic results show that our photometric samples are of a high degree of reliability, and that our method confers a higher success rate for identifying bona fide Sculptor stars than simple CMD-based selections (e.g., compare Figure 11a to Figure 4 of (Tolstoy et al. 2004); see also Papers VI and IX). However, it is worthwhile to investigate the origin of the remaining few sample interlopers found among the spectroscopic sample of Sculptor giant candidates.

First, our derived magnitudes are not perfect, so that there is some photometric scatter across our selection boundaries. Inspection of Figure 13 shows that a number of our false positives and false negatives are near the borders of our selection regions. The color and magnitude error bars in Figure 13 demonstrate that some of the false classifications can be explained by photometric errors. Table 6 gives the mean color and magnitude errors for the various samples used here for the analysis of the structure of Sculptor. The tabulated means show where potential vulnerabilities to photometric contamination are highest (e.g., on the lower RGB).

There are also some objects that lie more squarely within our selection limits that are not kinematically associated with Sculptor. These objects likely fall in one of the following categories: point-like galaxies (which are dominated by the light from giant stars or may have their magnesium features redshifted out of the DDO51 band), metal-poor field subdwarfs (which also have suppressed magnesium lines), or MW field giant stars. Our means of selecting against galaxies relies on morphological parameters output by PSF-fitting, but point-like galaxies like quasars and AGN may pass these criteria unabated. In fact, among our sample of RGB/RHB candidates we spectroscopically observed one quasar at $(\alpha, \delta)=$ $\left(01^{\mathrm{h}} 03^{\mathrm{m}} 33.71,-34^{\circ} 55^{\prime} 49^{\prime \prime} \cdot 2\right)$. But any galaxies in the sample would likely be revealed by extreme, non-Milky Way-like RVs.

According to the isometallicity lines given in Figure 5, metal-poor $([\mathrm{Fe} / \mathrm{H}] \leq-2.5)$ sub-dwarfs may also pass our 2CD selection. Following arguments given in Paper II, we should find $\lesssim 18$ such subdwarfs in our entire survey area (i.e., at a density of $\Sigma \lesssim 0.6 \times$ $10^{-3} \operatorname{arcmin}^{-2}$ ), and we certainly have fewer than this limit. Our selection criteria allow for selection of field giant stars that happen to have colors and magnitudes similar to the Sculptor RGB/RHB. Again, the contaminant surface density here is small given estimates 
from our control fields ( $\S 5.1)$. It is worth noting that Milky Way stars orbiting prograde about the Milky Way will, in general, have $v_{\text {hel }} \lesssim+35 \mathrm{~km} \mathrm{~s}^{-1}$ in this direction of the sky $\left([l, b] \sim[288,-83]^{\circ}\right)$. This can be seen, e.g., by the distribution of velocities for nonSculptor stars in Figure 4 of Tolstoy et al. (2004), which has a concentration of stars with these velocities. Only dynamically hot stars - stars with either retrograde orbits or with significant motions in the negative Galactic $Z_{G C}$ direction - will be expected to have higher $v_{\text {hel }}$. Several of our false positives are in this category. In addition, we find that for those false positives having MIKE echelle spectroscopy, the equivalent widths of the calcium infrared triplet lines are generally comparable to those of both Sculptor and Carina dSph members observed on the same run but less than other, more metal-rich stars also observed in the Carina field (more details are presented in $\mathrm{Mu} 05 \mathrm{~b}$ ). These velocity and chemical traits suggest that some halo stars are contributing to the false positives.

Still yet another explanation for at least some of the false positives is that they are actually Sculptor stars. We have already noted (§4.4) that the velocity dispersion of the dSph appears to be growing with radius, yet we used the same $\sigma_{v}$ rejection at all radii to exclude likely non-members. However, of the nine false positives in our spectroscopic sample we note that three lie within $15 \mathrm{~km} \mathrm{~s}^{-1}$ of our Sculptor velocity membership limits, six lie within $32 \mathrm{~km} \mathrm{~s}^{-1}$ and all but one of these are beyond 50 arcmin from the Sculptor center. At these velocities and considering their $(l, b)$ these six "false positives" must either be halo stars that are highly retrograde and/or that have a large Galactic $Z$ motion perpendicular to the disk; yet there are not comparable numbers of false positive stars with the opposite properties, as might be expected for a randomly populated, hot halo. It seems conceivable that at least some of these stars might be bona fide Sculptor giants, consistent with the notion that the velocity dispersion is growing with radius.

No matter their origin, in the end, all of these contamination effects will be accounted for as (a small) "background" in our analysis of the Sculptor radial density fits ( $(5.2)$, and we expect our high $S / B$ Sculptor giant candidate lists to provide a reliable means by which to map the structural properties of the Sculptor dSph. While we do not have a similar spectroscopic testing of the BHB samples, we shall show ( $\$ 5.3)$ that these stars yield very similar Sculptor morphological properties to the RGB samples. 


\section{DISTRIBUTION OF CANDIDATE SCULPTOR MEMBER STARS}

\subsection{Background Determination}

A proper assessment of the background is critical to proper determination of the density distribution of the low density dSph galaxies. This rings particularly true for the Sculptor dSph given the reviewed $(\S 1)$ history of attempts to determine its physical extent. Our goal here has been to attack the problem anew with a methodology aimed at substantially reducing the background level so that fractional errors in its estimation have significantly less effect on our morphological parameterization of the system, particularly in its tenuous outer parts. We will make a quantitative assessment of the resultant $S / B$ improvement below (§5.2.4), but a qualitative impression of our success is given by the CMD of non-selected stars shown in Figure 9c, which demonstrates the amount of (mostly) foreground material along the Carina RGB that has been removed from consideration, while the spectroscopic assessment of our contamination level (Table 5) shows that our residual contamination rate is very low to at least $M \sim 19(\sim 6 \% ; \S 4.5)$, and likely very good to even fainter magnitudes. Nevertheless, our contamination rate is not zero, and we now attempt to clarify how much residual "background" level remains so that we can establish the radial profile of Sculptor to the lowest possible densities.

In our previous papers (e.g., Paper II; Paper IV), estimation of the background to our RGB samples has been based on the assumption that, to first order, the densities of MW halo giants and $\mathrm{HB}$ stars roughly follow $R_{\mathrm{GC}}^{-3}$ laws, so that their contribution along a line of sight is roughly flat in magnitude. Under these circumstances, were one to shift the $\mathrm{RGB} / \mathrm{RHB}$ and BHB CMD selection criteria in magnitude (in the case of the RGB/RHB samples, this is done through the CMD of stars already selected to be giant candidates in the 2CD), the background contribution of these field stars should be approximately constant. For other classes of background contaminant (e.g., metal-poor disk disk subdwarfs with an exponential density law, halo dwarfs which are close enough that heliocentric distance is not a reasonable proxy for $R_{\mathrm{GC}}^{-3}$, and other disk stars with large photometric errors) the $R_{\mathrm{GC}}^{-3}$ assumption will be less correct, but the above method provides a reasonable first order estimate of the background level. ${ }^{11}$ Table 7 gives the results of shifting our adopted CMD selection regions in magnitude. The brightest magnitude offset (i.e. largest negative $\Delta M$ listed) is set by the

\footnotetext{
${ }^{11}$ Indeed, for some classes of contaminants - e.g., blue, main sequence turnoff dwarfs from the Galactic disk and thick disk which are not expected to lie in our Sculptor CMD selection region because of the extremely large implied distances from the Galactic plane - we will be overestimating their representation by shifting the CMD-selection region to brighter magnitudes. This situation counteracts the shallowing of the $R_{\mathrm{GC}}^{-3}$ assumption for halo MSTO dwarfs.
} 
saturation limit of the CCD frames; shifting the region to fainter magnitudes is precluded because of problems with survey incompleteness in this direction. When determining a background estimate with this method, one must "clear" those small $\Delta M$ offsets where Sculptor stars themselves contribute; for each sample in Table 7 the CMD offsets where background stars dominate are those below the horizontal line. Taking the average counts below these lines and dividing by the total area covered by each sample yields the estimated background number density, $\Sigma_{\mathrm{CMD}}$, given in the final line of Table 7 along with its Poissonian error.

A second method by which we can estimate the background is by determining the number of "Sculptor giants" selected by our 2CD and CMD criteria but at large distances from the Sculptor core. Unfortunately, our Sculptor radial surface density plots never converged to a constant background level even as we continued to add spatial coverage at ever larger radii. To remedy this problem we observed four background "control" fields significantly separated (by $5^{\circ}$ ) from the center of the dSph in each of the cardinal directions (Figure 1a). At such remote locations with respect to Sculptor (with $r_{\text {lim }}$ estimated at 76.5 by IH95) it is less likely that we will see a significant contribution from Sculptor stars. These four fields were taken during the 23 October 2001 observing run (see Table 1) and are of similar quality to our normal survey fields, though their magnitude limits vary. Table 8 gives the background densities in our complete list of giants (just the 2CD selection) and in each of our magnitude limited samples for each 353.85 arcmin $^{2}$ control field; no data are given when the fields were insufficiently deep for proper comparison with a particular, magnitude-limited sample. Confidence limits for a Poissonian probability distribution are given at the $90 \%$ and $68 \%$ levels. Unfortunately, three of the four control fields were insufficiently deep in $T_{2}$ to characterize accurately the background number density of our BHB samples. The final column gives the average control field background level, $\Sigma_{\mathrm{CF}}$.

Table 8 illustrates several things. First, the background densities derived by this independent method are also extremely low; only 0 , 1, or 2 stars were found for any sample in any field. This reflects both the efficacy of the Washington+DDO51 technique to throw out foreground dwarfs as well as the high, $b=-83^{\circ}$ latitude of the Sculptor system, which limits the number of MW contaminants overall. The 2CD giant selection throws out 95-98\% of the total stars in the control fields and $98-100 \%$ of the total is thrown out when both the 2CD and CMD selections are imposed. Second, the background densities estimated from the control fields are similar to those found with the "CMD offset" method. Although the background densities derived with the latter method are slightly smaller than those determined by the control fields, they are well within the confidence limits (though we acknowledge that the confidence limits on the control field densities are large relative to the actual values because of the small number statistics from which they are derived). Finally, the background 
estimates obtained with either the method illustrated in Table 7 or that in Table 8 are consistent (within Poissonian errors) with the spectroscopic determination of a small ( $\sim 5 \%$ ) "false positive" rate in our RGB samples to either $M=19.0$ or $M=20.3$ (Table 5 ; §4.5).

\subsection{Radial Plots}

\subsubsection{Fitting Functions}

We fit our Sculptor density distribution with both a single component King model and with a power-law with a flat core (PLC) model, the two functions used by Kleyna et al. (1998) in their study of the Ursa Minor dSph. The fitted King functions define the stellar surface density, $\Sigma_{\mathrm{K}}(Q)$, as

$$
\Sigma_{\mathrm{K}}(Q)= \begin{cases}k\left\{\left(1+Q^{2}\right)^{-\frac{1}{2}}-\left[1+\frac{r_{\lim }^{2}}{a^{2}(1-\varepsilon)}\right]^{-\frac{1}{2}}\right\}^{2}+\Sigma_{b}, & \text { if } Q^{2} \leq \frac{r_{\lim }^{2}}{a^{2}(1-\varepsilon)} \\ \Sigma_{b} & \text { otherwise }\end{cases}
$$

where

$$
\begin{aligned}
Q^{2} & =\frac{X^{2}}{a^{2}}+\frac{Y^{2}}{a^{2}(1-\varepsilon)^{2}} \\
X & =\left(\xi-\xi_{0}\right) \sin \theta+\left(\eta-\eta_{0}\right) \cos \theta \\
Y & =-\left(\xi-\xi_{0}\right) \cos \theta+\left(\eta-\eta_{0}\right) \sin \theta
\end{aligned}
$$

$k$ is the normalization constant, $r_{\text {lim }}$ is the King limiting radius, $a(1-\varepsilon)^{\frac{1}{2}}$ is the semi-major core radius, $\varepsilon=(1-b / a)$ is the system ellipticity, $\theta$ is a rotation angle on the sky (where the normally defined position angle, $P A$, is given by $90-\theta)$, $\xi$ and $\eta$ are the stellar coordinates deprojected to the tangent plane to the sky, and $\Sigma_{b}$ is the fitted background density. The PLC model is given by

$$
\Sigma_{\mathrm{PLC}}(Q)=\frac{M(\nu-1)}{\pi a^{2}(1-\varepsilon)\left(1+Q^{2}\right)^{\nu}}+\Sigma_{b}
$$

where $M$ is the total number of stars, $\nu>1$ is the power-law fall off, and the other parameters carry the same definition as in the King model. Best-fitting parameters were calculated using Bayesian and maximum likelihood techniques while errors are estimated using a Bayesian approach in conjunction with a Markov Chain technique. The fitting algorithms are explained in greater detail by Ostheimer (2002) and Ostheimer et al. (2005). 


\subsubsection{Initial Profile Fitting Results}

The best-fitting King profile parameters to our different magnitude-limited samples are given in Table 9 along with previous literature values for comparison. The best-fitting PLC profile parameters are given in Table 10. Tables 9 and 10 also give the error-weighted means of the model parameters derived from the four different stellar samples analyzed. Note that the error on the quoted mean values will be somewhat under-estimated because the different samples are not completely independent of one another. The best-fit model profiles are shown against the stellar densities in Figure 14. A formal model is not fit to the $M \leq 21.0$ sample because the center of the galaxy is not represented in this sample. Instead, this sample is modeled by the functional parameters fit to the $M \leq 20.3$ sample but scaled to the same average density of stars as the $M \leq 20.3$ sample in fields where both samples are represented; the result is overplotted on the $M \leq 20.3$ sample as open circles.

Table 9 shows the considerable agreement of all our samples in each derived morphological parameter and their general consistency with most of the literature values (though see §5.2.4). Figure 10 shows the King limiting radius for the various samples, and the differences are nearly imperceptible. The consistency between the model parameters found for the RGB/RHB and BHB samples is especially gratifying given the differences in the selection methodology used for each sample and the expected relative purity of the BHB sample. The only parameters with variations inconsistent with the formal errors are the center co-

ordinates. However, given the flatness of the surface density in the Sculptor core and our uneven sampling of Sculptor with position angle, the profile center is not well constrained; the observed $\sim 1.5$ center coordinate variations are, therefore, not surprising. To check for possible correlations between parameters presumably more susceptible to the uneven $P A$ coverage of the data, the fitting program was also run with the center coordinates, $P A$, and $\varepsilon$ fixed to their tabulated mean values; no significant changes in the derived $r_{c}$ and $r_{\lim }$ from the best-fit values in Table 9 were found.

\subsubsection{Background Level Effects on the Radial Profile}

Comparison of Tables 9 and 10 reveals an important difference between the King and PLC fits: The model-estimated backgrounds are significantly larger in the King fits than they are in the PLC fits, and they are larger than those found by the background estimations described in $\S 5.1$. The explanation lies in the mismatch between the actual distribution of Sculptor stars and a King profile. This difference is portrayed by (1) the distributions of stars in Figure 10, which show an "extratidal" population with declining radial density outside the best-fit King limiting radii, and (2) by the poor fit of the King profile to the density 
distribution at the largest radii in Figure 14. The issue of whether a King profile is physically meaningful for a dSph system aside ${ }^{12}$, the adopted, one component King function (Equation 6) simply is not mathematically suited to accommodating the approximately $r^{-2}$ decline of the Sculptor surface density at large radii. When allowed to "find" the background on its own, the King profile fit averages the "extratidal" Sculptor stars into the mean background level. On the other hand, the PLC fit, which is designed to accommodate a power law decline in density, obtains background estimates much closer to those estimated from the control fields and the "CMD offset" method. On the other hand, the PLC models fail to account for the apparent inflection point, or "break", in the radial profile near $60^{\prime}$, where the slope of the profile apparently changes.

To remedy this problem, we next attempt King function fits where the backgrounds are fixed to our measured $(\S 5.1)$ values. Since the only measurable background density in the control fields is for the $M \leq 20.3$ and $M \leq 21.0$ samples, only for these do we use $\Sigma_{\mathrm{CF}}$ to estimate the background; for the other samples we use $\Sigma_{\mathrm{CMD}}$. We are then, in effect, always using the larger of the two background estimates. As expected, however, the results are unsatisfactory in that they now result in clearly exaggerated tidal radii (Figure 15); in attempting to compensate for an observed density profile that now clearly shows a significant density at large radii, the fitting code must inflate $r_{\text {lim }}$.

On the other hand, the density profile within the break radius is well described by the initial King profile fit, which is largely insensitive to the low density outskirts of our maps due to the overestimated background level. This is demonstrated by Figure 16, which compares the original King profile fits to the the true density distributions accounting for the measured background levels. The measured background levels are subtracted from the points plotted and, as in Figure 14, are shown to highlight where our data reach $S / B=1$. In the case of the BHB stars the $S / B=1$ limit is apparently beyond the limits of our main Sculptor survey. As Figure 16 demonstrates, the central regions of the dSph are, in fact, well described by the King profiles in Table 9 down to approximately three orders of magnitude below the central density; for this reason, we feel it is justified to adopt the parameters found in Table 9 as appropriate descriptors for the central Sculptor populations. However, a diffuse "break" population dominates the density beyond the King limiting radius. Like other dSphs we have studied in a similar manner (Paper II; Paper IV; Paper IX; Paper $\mathrm{X})$, Sculptor can be described by a "King +Break" $(\mathrm{K}+\mathrm{B})$ profile - a characteristic seen in

\footnotetext{
${ }^{12}$ Because the crossing time/two-body relaxation time for a dSph galaxy is long compared to globular clusters, it is not clear that a King profile, which was designed to describe these compact, higher density systems, should be an appropriate physical model for dSphs. We return to the physical interpretation of the Sculptor density profile in $\S 6$.
} 
models of tidal-disrupting satellite galaxies (Johnston 1998; Mayer et al. 2001, see §6). This break is compared to a least-squares fitted and $\Sigma \propto r^{-2}$ power-laws in Figure 16.

From Table 10 we see that the PLC profile parameters are fairly consistent except in the case of the $T_{2} \leq 19.9$ sample where the small sample size is apparently causing difficulty in the fit. A notable characteristic of the PLC fits is their $\nu \sim 2$ index, a value for which the PLC model reduces to a projected Plummer model. As we pointed out above and show in Figures 14 and 16, while the PLC fits seem to give better matches to the data over all radii, they do not account for the profile break and increasingly fail to match the measured density at large radii. The $\nu \sim 2$ indices derived with the PLC fits correspond to $\sim r^{-4}$ density declines - faster than the $\sim r^{-2}$ declines that provide a better fit to the outer densities (e.g., see left panels in Figure 16). This particular failing of the PLC models is illustrated, for example, by the fact that in the $T_{2} \leq 19.9$ case, the PLC-fit "background" is nearly an order of magnitude higher than that calculated (Table 7 ).

To put a more quantitative measure on the relative quality of the fits using the different backgrounds and surface density profiles, we have calculated a simple $\chi^{2}$ statistic $^{13}$ using the data presented in Figures 14 and 16; the results are tabulated in Table 11. For the "King+break" ("K+B") profiles, the break population is modeled by both the least-squares fitted and an adopted $r^{-2}$ power-law. The starting radius of the power law (given in Table 11), $r_{\text {break }}$, is that which yields the best $\chi^{2}$ value; the normalization is determined by the value of the model King profile at $r_{\text {break }}$. In general, when compared to the simple King profile (without a break component), the PLC model proves to be a better fit when using either the model or measured $\Sigma_{b}$. However, in two out of the four cases determined using the measured $\Sigma_{b}$, a K+B profile provides a better fit to the data than does the PLC profile. Moreover, the $\mathrm{K}+\mathrm{B}$ profile provides a better fit when compared to a simple King profile even when the inflated, model $\Sigma_{b}$ is used; the one exception is the $M \leq 19.0$ sample, which has only one point that could be confidently considered part of a break population. The overall consistency of $r_{\text {break }}$ across all samples is encouraging given that it is independently determined for each sample.

While providing comparable fits to the overall distribution, the failings of the PLC models to match the observed features (density and inflection point) at large radii are the primary reasons why, in the end, we prefer the $\mathrm{K}+\mathrm{B}$ profile description for the Sculptor morphology. But for either the PLC or $\mathrm{K}+\mathrm{B}$ description, a clear and relevant point is that

\footnotetext{
${ }^{13}$ Note that Ostheimer's (2002) fitting routine does not minimize this $\chi^{2}$ to determine best-fitting sets of parameters. The a posteriori-calculated $\chi^{2}$ statistic is used simply as a relative figure of merit to intercompare models.
} 
Sculptor extends to the edge of our main survey field (150' from the Sculptor center), within which there does not seem to be a "Sculptor-free", background region.

\subsubsection{Comparison to Previous Results}

Our derived King profile fit parameters (Table 9) — in particular $P A, \varepsilon$, and $r_{c}$ - are in general agreement with previously measured values for Sculptor. The most important difference appears to be that we consistently find King limiting radii near the upper end of the previously reported range and twice that of the lower estimates, most notably the recent CCD study by W03. Only the Eskridge (1988b) value for the limiting radius is larger than ours. We find a large King limiting radius despite our adoption of the King parameters derived with the artificially inflated, model-fit backgrounds. As found in previous attempts to fit the Sculptor radial profile (see $\S 1$ ), we also find that use of the measured background level increases our derived King limiting radius and, also, reduces the quality of the fit at smaller radii as shown in Figure 15. Moreover, we find a sizable excess density beyond the King limiting radius when using either the measured or the model-fit background. As Tolstoy et al. (2004) have done, we have also spectroscopically confirmed Sculptor velocities for several of these outliers.

The origin of the difference in our measured King radius compared to that found in other studies is not likely due to differences in the depth of the data. Our actual imaging limit is comparable to that of all of the previous studies, except the W03 study, which reaches $\sim 2.5$ magnitudes deeper than our data. Instead, the difference in model fits probably lies in the significantly better $S / B$ delivered by our methodology, which allows us to explore the dSph to substantially larger radius with much greater sensitivity. All previous Sculptor surveys employed simple statistical starcounts to map the dSph. Figure 17 demonstrates the significant improvement gained by our approach: We compare the equivalent Sculptor radial $S / B$ (given by $\Sigma_{\mathrm{K}} / \Sigma_{b}-1$ ) profiles delivered by our data when we (1) adopt the traditional, simple starcounting approach to measure the radial density (i.e., all stars detected are used to determine both $\Sigma_{\mathrm{K}}$ and $\Sigma_{b}$ ), (2) mimic a "CMD-filtered" starcount analysis ${ }^{14}$ whereby only stars meeting our CMD criterion for RGB/RHB selection are used to measure the density, and (3) adopt both our 2CD and CMD selection criteria to find only the most reliable Sculptor giant candidates, as discussed in $§ 3$. In all cases, we use the $M \leq 20.3$

\footnotetext{
${ }^{14}$ As discussed in $\S 1$, this has become a popular method of mapping stellar systems to large radii (see, e.g., Grillmair et al. 1995; Odenkirchen et al. 2001, 2002; Rockosi et al. 2002). One filters the starcounts to those sources that lie near the primary CMD locations occupied by stars in the stellar system.
} 
sample. As may be seen, making use of color-magnitude information to tune the starcount selection to the Sculptor RGB/RHB does improve the $S / B$ of a survey by about a factor of $\sim 4$ over simple star counting techniques (as used by, e.g., IH95 and W03). But even this CMD filtering falls far short of the nearly 200 times lower background achieved by using the two-criteria, Washington+DDO51 technique to weed out foreground dwarf stars first. The radius at which the profile density sinks to the associated background is $\sim 20^{\prime}$ using simple starcounts and $\sim 40^{\prime}$ using the CMD selection for RGB/RHB stars, but expands to beyond $100^{\prime}$ for the 2CD and CMD-filtered, giant star candidate sample. Note, because Sculptor is situated near the SGP, it represents the best situation with regard to foreground dwarf contamination; thus, use of the Washington+DDO51 approach should have a greater impact on the study of lower latitude systems.

The actual situation with regard to previous Sculptor studies is actually worse than demonstrated in Figure 17. For example, in both IH95 and W03 the authors did not discriminate between stars and background galaxies, but counted all sources in their survey. However, as demonstrated by, e.g., Reid \& Majewski (1993), at $M \gtrsim 20$ galaxies actually outnumber stars at the (North) Galactic Pole by approximately 5:1. In the Sculptor field near the SGP, the background in our star counts profile becomes 2.1 or 1.6 times greater if we make no image morphology cuts or do not select against likely galaxies (large $\chi$ at faint $M$ ), respectively. Were one to probe to deeper magnitudes in search of more dSph-rich regions of the CMD (e.g., beyond the MSTO), one might be thwarted by the fact that the galaxy contamination rate not only rises faster than the contribution from field star contaminants, but it rises faster than the typical luminosity function of the dSphs themselves (see, e.g., the luminosity functions for old stellar populations summarized in Figure 5 of Reid \& Majewski 1993). Obviously, such contamination will have its greatest impact on the lowest density regions of the dSph, but even the core regions of the IH95 and W03 studies have been significantly impacted by the lack of galaxy pruning. The quoted source counts of IH95 are about an order of magnitude greater in the Sculptor core than we find from the sum of all our stellar detections after removing morphologically-discriminated galaxies. Yet the IH95 study only probes slightly fainter. On the other hand, the W03 survey probes 2.5 mag deeper than ours, so they would be expected to have a higher source density in the core. However, assuming the luminosity function of Sculptor is approximately like that of a globular cluster (see, e.g., Eskridge 1988a), we find an expected factor of $\lesssim 20$ in Sculptor stars gained by the increased depth of W03, which cannot account for the W03 factor of $\sim 45$ greater central surface density compared to our morphologically-discriminated counts in the Sculptor center.

We can also directly compare the $S / B$ of our Washington+DDO51 filtered counts to those adopted by the IH95 and W03 studies. Using Table 3 from IH95 and Figure 5 from 
W03, we find peak values of $S / B \sim 20$ and $S / B \sim 1000$ for these studies, respectively. In contrast, we have a peak $S / B \sim 8000$ for our data (Figure 17). These $S / B$ comparisons are slightly misrepresentative because the backgrounds in these previous surveys were actually calculated at smaller radii, where Sculptor stars contribute.

Finally, it is worth pointing out that tracing Sculptor by its BHB stars yields results with comparable $S / B$ to those from our Washington $+D D O 51$ giant star mapping technique. Given the expense of obtaining the narrow-band DDO51 photometry, it would seem that simply mapping BHB stars with broadband photometry may be a more effective means to explore the extended structure of the Sculptor dSph. However, we stress that our longterm goal is not only to determine the spatial characteristics of Sculptor and other dSph systems, but to derive their large-scale dynamics, and giant stars are much more accessible spectroscopic targets than are BHB stars at the same distance. In this vein, Figure 17 has important bearing on the prospects for spectroscopic studies of the Sculptor dSph at very large radii: By a semi-major axis radius of $\sim 200^{\prime}$ (i.e., 2.5 King limiting radii), less than $5 \%$ of $M \leq 20.3$ stars in the RGB part of the CMD are actually part of Sculptor, which has a density of $\lesssim 4 \mathrm{deg}^{-2}$ (Figure 16). Thus, even with wide field, multifiber spectroscopy, it will not be possible to explore Sculptor RGB stars at such large radii efficiently if the input sample is selected only as "RGB-like" stars in the CMD. (Note that while they are easier to find as "needles in the haystack", BHB stars have a sky density an order of magnitude smaller than that of the RGB, and, of course, they are fainter.) It is in this very low regime, where our relatively reliably identified samples of $\left(M, T_{2}, D D O 51\right)$-selected RGB stars are therefore particularly valuable.

\subsection{Two-dimensional Structure}

Break populations are just one feature associated with satellites undergoing tidal disruption. Discernible tidal tails and internal substructure (e.g., lumpiness and S-shapes) are others. W03 point out the possibility of a tidal tail extending to the southeast at a semimajor axis radius of $30^{\prime}$ to $40^{\prime}$ (note that this is "extratidal" for them, but well inside our newly determined King limiting radius). Moreover, both Eskridge (1988c) and IH95 report an increasing Sculptor ellipticity with radius, which is an effect one would observe if tidal arms were forming (Johnston, Choi \& Guhathakurta 2002).

Our high $S / B$ Sculptor maps offer the opportunity to look for these previously noted features. Figure 18 plots contours of the inner regions of all but our $M \leq 21.0$ sample. We also over-plot the $M \leq 20.3$ and $T_{2} \leq 19.9$ samples (Figure 18e) to show the strong correspondence between the spatial distribution of these two samples where the spatial coverage 
overlaps. Finally, to give our most complete representation of Sculptor, we have plotted contours derived from the combined $M \leq 20.3$ and $T_{2} \leq 19.9$ samples (Figure 18f).

Figure 18 displays interesting aspects of the central distribution of Sculptor stars. An increase in the ellipticity of Sculptor with radius is apparent from both the $M \leq 19.0$ and $M \leq 20.3$ contour plots: While the dSph is more or less round within about $20^{\prime}$, it becomes extended in the east-west direction at larger radii, as has been previously noted (e.g., IH95). Moreover, while these maps show a slightly higher density toward the northeast in our RGB/RHB samples, with none of our samples can we confirm a Sculptor extension to the southeast as reported by W03. As one possible explanation for the difference we draw attention to the comment made by W03 that the interpretation of possible tidal extensions in their data might have been influenced by the fact that their contour maps made were made without benefit of star/galaxy discrimination in their source catalog; we have already discussed (§5.2.4) how significant a contribution galaxies are likely to be to the W03 source counts. On the other hand, the Sculptor maps of IH95 (see their Figure 1f) also suggest an extension of Sculptor to the northeast albeit at lower significance than we see in our maps. It is interesting that this extension is along the direction of the Sculptor proper motion as determined by Schweitzer et al. (1995), as might be expected for tidal debris.

As a further demonstration of the increasing ellipticity with radius and to assess the apparent northeast excess (which would imply isophotal twisting), Figure 19 maps the azimuthal trends of the mean Sculptor density for different radial annuli for both the $M \leq 19.0$ and $M \leq 20.3$ samples. For this analysis, the radial annulus sectors are shaped according to the ellipticity parameters derived from the mean fit to the entire galaxy (line 5 in Table 9) and radial plots were determined for azimuthal bins. If the model ellipse is a perfect fit at a particular semi-major axis radius, there should be no azimuthal trend in density and all sectors should have the model surface density. If, on the other hand, the galaxy is more or less elliptical than the model at a given radius, then the density will show variations from the model: In the case of ellipticity changing at a fixed PA, one will find an increase in the density over the model along the semi-major axis $P A$ s and deficits along the semi-minor axes, whereas if it is rounder it will show the opposite trend. Any isophotal twisting (as, for example, one might see with the onset of well-defined tidal tails) will be evidenced as azimuthal shifts of such features with radius. The radial bins in Figure 19 are logarithmically and coarsely spaced to improve the signal-to-noise of the points. Our ability to interpret this figure is complicated by the small area represented by annuli at short radii and incomplete survey coverage at larger radii. Nevertheless, Figure 19 seems to confirm the impression that the galaxy changes shape from rounder than our global fitted shape to more elongated than our global fitted shape between $r=22^{\prime} \cdot 7$ and $r=40$ '. 8 . The data do not show obvious signs of isophotal twisting, but are of insufficient quality to do so reliably. 


\subsection{Spatial Metallicity Variation}

The idea of a metallicity spread within Sculptor has long been recognized from photometric studies of its giant branch, (Kunkel \& Demers 1977; Norris \& Bessell 1978; Smith \& Dopita 1983; Da Costa 1984; Eskridge 1988a), via studies of its RR Lyrae population (Goldsmith 1993; Kaluzny et al. 1995), and through spectroscopic analysis (Tolstoy et al. 2001; Shetrone et al. 2003; Tolstoy et al. 2003, 2004). Majewski et al. (1999) have argued that this spread is really a bimodal population distribution described by populations with $[\mathrm{Fe} / \mathrm{H}] \sim-2.2$ and $\sim-1.4$; it was suggested to possibly be trimodal by Grebel, Roberts \& van de Rydt (1994). Spatial gradients in the Sculptor populations (whether due to a true gradient or as a change in mixing ratio of a bimodal metallicity distribution) have also previously been explored (Light 1988; Da Costa et al. 1996; Hurley-Keller, Mateo \& Grebel 1999; Majewski et al. 1999; Harbeck et al. 2001; Tolstoy et al. 2004). Given our larger area coverage of Sculptor than in previous studies and the more reliable catalog of giant stars we have created, it is worthwhile to re-evaluate the spatial distributions of stars with apparently different metallicities.

Since we have no better indicator of intrinsic metallicity for the bulk of our giants other than their position in the CMD, we divide our RGB populations into "metal-rich" (red) and "metal-poor" (blue) halves by fitting a fourth order polynomial to the center of the CMD locus of the RGB as shown in Figure 20. This division into metallicity halves is reasonable whether or not Sculptor's metallicity distribution function is bimodal or more continuous. Figure 21a shows the cumulative radial distributions of these two RGB halves for our $M \leq 19.0$ sample, where the stars have been binned along the abscissa in terms of their equivalent semi-major axis distance from the Sculptor center. To make these distributions, we have fixed the Sculptor center, $P A$, and $\varepsilon$ to that of the mean of our best-fitted King models (Table 9). The separation of the blue and red RGB distributions is clear, with the more metal-rich Sculptor population being more centrally concentrated (as has been found by previous studies). The difference in these two distributions is quantified using a Kolmogorov-Smirnov (K-S) test and the results are given in Table 12. This table provides the effective number of data points $\left(N_{e}\right)$, the K-S statistic $(D)$, and the K-S significance level $(\mathrm{KS} \%)$, as calculated by the algorithms provided by Press et al. (2002). The results of this test show a strong disagreement with the null hypothesis that these two spatial distributions are the same.

We repeat the analysis with deeper probes of the RGB, but here we meet with a population mixing problem due to contamination of the "blue" RGB by the Sculptor RHB. The RHB population properly belongs to the red (metal-rich) RGB (e.g., Majewski et al. 1999), and therefore, a simple RGB division will tend to wash out the difference in the distributions 
between the red and blue populations. We have removed the CMD regions of our $M \leq 20.3$ sample that are clearly dominated by RHB stars, but have left in the sample that part of the CMD where the RHB and the blue RGB overlap (compare Figures 7 and 20). This residual RHB contamination of the blue RGB sample will cause a modest dilution of the red/blue spatial distinction. Indeed, while we find the distributions of the blue and red stars in Figure 21b to be significantly different, they are slightly less so than in the $M \leq 19.0$ case (note the difference in K-S probabilities in Table 12).

Obviously, we expect the BHB sample, which belongs to the more metal-poor Sculptor population, to track the spatial distribution of the blue RGB sample. We test this by comparing the $T_{2} \leq 20.3$ sample to the two $M \leq 20.3$ samples in Figure 21c. To do this fairly, we have excluded sky regions of the $M \leq 20.3$ samples that are not included in the $T_{2} \leq 20.3$ sample. It is hard to distinguish between the BHB and blue RGB samples, as might be expected (Figure 21c), and the distributions are identical at the $99 \%$ level as given by the K-S test. On the other hand, the BHB distribution is significantly different than that of the red RGB sample.

To further illustrate their different distributions, the blue and red RGB distributions have been individually fit with King model profiles. The best-fitting parameters are given in Table 13, the individual radial profiles illustrated in Figure 22 and the sky distributions shown in Figure 23 together with their best-fitting King limiting ellipses. The background, $\Sigma_{\mathrm{CMD}}$, for each radial profile has been determined using the "CMD offset" method for each half of the RGB. Again, the greater concentration of the metal-rich population is apparent by comparing, for example, the differences in the core/limiting radii in Table 13 . However, our new, wide area data now show this spatial difference to be maintained to the nominal $80^{\prime}$ limiting radius of the system and beyond. While both metallicity populations apparently contribute to the "break" population beyond this radius, the metal-poor population density is greater than the metal-rich population there by an order of magnitude. The radial gradient in the make-up of Sculptor is also demonstrated in Figure 22 by the variation of the fractional population balance parameter, $(B-R) /(B+R)$, where $B$ and $R$ are the number of blue and red RGB stars within an elliptical annulus. Interestingly, the small east-west extension of the metal-rich population as seen in Figure 23 leads to the finding of a greater ellipticity compared to that for the metal-poor population.

\section{SUMMARY AND DISCUSSION}

We have undertaken the most sensitive morphological survey of the Sculptor dSph system to date. Our ability to increase the signal-to-noise of the measured Sculptor structure is 
due to our orders of magnitude improved signal-to-background, $S / B$, over that in previous work. By using the Washington+DDO51 photometric system to create maps of Sculptor giant stars, and by taking advantage of the strong contrast of Sculptor BHB stars to Galactic field stars of the same color, we are able to reduce substantially the backgrounds, $B$, in our maps and thereby track the radial profile of very high probability Sculptor stars to the largest angular extents $\left(\sim 150^{\prime}\right)$ explored for this system thus far. At the edge of our survey area, the primary limit on our ability to resolve detailed two-dimensional structure on the scale of tens of arcminutes is the quantization noise of individual BHB and RGB sources at low densities.

These data have been applied to a variety of analyses to obtain the following results:

1. Through spectroscopic follow-up of 157 stars we verify that our Washington+DDO51 methodology to select candidate Sculptor RGB stars actually produces true Sculptor members with a $94 \%$ reliability rate. Thus our Sculptor RGB candidates can be trusted to give an accurate tracing of the structure of the Sculptor system. Admittedly, this reliability rate comes at some expense in terms of completeness, and we have identified some radial velocity members of Sculptor with color-color-magnitude combinations that place these stars just outside our conservative selection criteria.

2. The Sculptor structural parameters are derived anew. Both simple King models and Power Law + Core (PLC) models provide unsatisfactory descriptions of the full Sculptor density distribution over the full range of our survey. Neither can properly account for a "break" to a shallower, approximately $r^{-2}$ radial density fall-off near a semimajor axis radius of $\sim 60^{\prime}$. On the other hand, the Sculptor radial profile is well-fit by a King profile within this radius, so that, as we have found for other dSph systems we have explored (Paper II; Paper IV; Paper X; Majewski et al. 2003), Sculptor is well-described by a "King + Power Law" function. Once adopting this prescription to account for the extended break population around Sculptor, we find central King model parameters that are within the range of those found previously by other studies, but an $r_{\text {lim }}=79.6 \pm 3$ '.3 King limiting radius that is at the high end of those previously reported; most notably, our limiting radius is nearly twice that found by the recent, deep CCD starcount survey by W03. We argue that such discrepancies arise from substantially higher contamination by both foreground stars and background galaxies in the previous surveys, which limited their effective signal-to-noise, particularly in the more diffuse outer regions of Sculptor. As a cautionary measure, we assess the dependence of our own derived King radius on adopted background. We consistently find $r_{\text {lim }} \approx 80^{\prime}$, despite allowing our fitting program to use an inflated background that is at least a factor of two, and up to an order of magnitude, larger than the background 
measured in control fields $5^{\circ}$ from the Sculptor center.

3. We confirm the existence of an extended component to the Sculptor system beyond the $\sim 80^{\prime}$ King limiting radius, and map this break population to the $150^{\prime}$ extent of our main survey area. An exorbitantly large background density would need to be adopted to eliminate this excess population at large radii. Instead, two independent methods used to measure our background levels agree that they are very low, and demonstrate that we detect the Sculptor break population at a $S / B \sim 3$ at the $150^{\prime}$ limit of our survey, and with $S / B \sim 10-40$ (depending on which sample of stars we use) near the break radius. Given this degree of significance, there can be no doubt that this break population is real. Moreover, the break population is seen with both RGB/RHB and BHB stars, and we have at least three (see §4.6) stars in the break population spectroscopically confirmed (Tolstoy et al. 2004, report an additional two). We stress this point because our previous detection (Paper II) of a break population around the Carina dSph using the same methodology has been questioned by Morrison et al. (2001) and W03 (but has been shown to be real in Paper VI and again in Mu05b).

4. The contrast of Sculptor RGB stars in its break population with respect to other field stars at the same color and magnitude are so low that simple strategies to pick such stars out of the CMD for spectroscopic follow-up will result in relatively low rates for uncovering actual Sculptor giants - for example, less than 5\% at $2.5 \mathrm{King}$ radii. Plowing through large numbers of stars with multifiber spectroscopy is not necessarily the best solution to probing the dynamics of Sculptor RGB stars at large radii, since the density of $M \leq 20.3 \mathrm{RGB}$ stars is $\lesssim 4 \mathrm{deg}^{-2}$ at $2.5 \mathrm{King}$ limiting radii. In contrast, at the same radius our Washington+DDO51 Sculptor RGB sample is expected to increase the success rate for finding bona fide Sculptor stars by more than an order of magnitude, and at a density efficiently probed with single slit spectroscopy. We hope to undertake this type of follow-up work, and have presented a successful proof of concept with results using the Magellan telescope and MIKE spectrograph here.

5. The two-dimensional structure of Sculptor shows evidence for a change in ellipticity with radius, from rounder to more elliptical, as previously reported by Eskridge (1988c) and IH95. Our deeper data sets also show an extension from Sculptor to the northeast along the direction of the Schweitzer et al. (1995) proper motion vector. This feature, lying between $20^{\prime}$ and $40^{\prime}$, is also seen at lower significance in the contour plot by IH95. We do not, however, confirm the extended feature to the southeast reported by W03.

6. Preliminary dynamical results using a total of 134 Sculptor stars ranging out to $\sim 0.6$ King limiting radii show a mean Sculptor velocity of $\left\langle v_{\text {hel }}\right\rangle=110.43 \pm 0.79 \mathrm{~km} \mathrm{~s}^{-1}$, a global velocity dispersion of $\sigma_{v}=8.8 \pm 0.6 \mathrm{~km} \mathrm{~s}^{-1}$, and no evidence for rotation. The 
radial trend of the velocity dispersion for our Sculptor stars is found to be relatively flat, however, as found in other dSph systems (e.g., Mu05b), Sculptor may show a rise in velocity dispersion beyond $\sim 0.4 r_{\text {lim }}$. In Mu05b we show how such a profile is one signature of tidally disrupting satellites on very radial orbits.

7. When the Sculptor RGB is divided into red and blue halves, representing predominantly more metal-rich and more metal-poor Sculptor populations, a clear difference in spatial distributions is observed. The more metal-rich giant stars are more centrally concentrated and, interestingly, more elliptically-distributed, than the metal-poor giants. The distribution of BHB stars closely follows the distribution of blue RGB (metal-poor) stars, as expected. These metallicity-dependent differences in spatial distribution echo previous findings (Light 1988; Da Costa et al. 1996; Hurley-Keller et al. 1999; Majewski et al. 1999; Harbeck et al. 2001; Tolstoy et al. 2004).

Spatial and dynamical characteristics of the Sculptor system reported here (e.g., the clearly detected break population, signs of increasing ellipticity with radius, and a rising/flat velocity dispersion profile) are classic signatures of a dwarf satellite undergoing tidal disruption. In this interpretation, the Galactic gravitational tides are stretching Sculptor at large radii internal to tidal radius, and stripping stars that extend into a break population beyond the nominal King limiting radius (which might then actually correspond to the tidal radius of the system). $\mathrm{K}+\mathrm{B}$ density profiles are consistent with model expectations for the appearance of a tidally disrupting dwarf galaxy in a MW-like potential (Johnston 1998; Mayer et al. 2001), where the break population represents unbound stars. Unbound stars at all radii can inflate the velocity dispersion and lead to the observed flat velocity dispersion profile (e.g., Kroupa 1997, Mu05b).

On the other hand, breaks within the tidal radius of a disrupting system have also been found in some N-body models explored by Johnston et al. (2002). Thus, Sculptor could be tidally disrupting, and still have the observed break population be bound. On the other hand, if one calculates where the tidal radius of Sculptor should be from its mass relative to that of the MW one finds that it is close to, or less than, the observed King limiting radius assuming a Sculptor mass of $1.5 \times 10^{7} \mathrm{M}_{\odot}$, consistent with an assumed $M / L=10.9$ in solar units $(\mathrm{IH} 95)^{15}$. A characteristic of all of the numerous orbiting satellite models

\footnotetext{
${ }^{15}$ Using the current Sculptor Galactocentric distance as an approximate orbital semi-major axis distance ( $a=79 \pm 4 \mathrm{kpc} ;$ Mateo 1998) and assuming a low orbital eccentricity ( $e \lesssim .1$ ), Equation 1 by Burkert (1997) yields a tidal radius $r_{t} \sim 65^{\prime}$ for a Sculptor mass of $1.5 \times 10^{7} \mathrm{M}_{\odot}$. A higher eccentricity $(e \sim 0.5)$ orbit yields a smaller tidal radius, $r_{t} \sim 40^{\prime}$. The Schweitzer et al. (1995) orbit determined for Sculptor implies $e=0.25$, between these two values.
} 
presented in the Johnston et al. (2002) study is an increase in the isophotal ellipticity as a function of radius. This increase in ellipticity is greater for those satellites on more circular orbits. Johnston et al. (2002) give observational signatures that help in determining the physical process behind the development of the structures seen in their models. They define the radius where the ellipticity of an originally circular model satellite becomes $>0.02$ as $r_{\text {distort }}$ and the innermost radius at which the change in the slope of the density power-law becomes $>0.2$ as $r_{\text {break. }}$. Their simulations show that if $r_{\text {break }} / r_{\text {distort }}>1$ and the slope of the power law beyond $r_{\text {break }}\left(\gamma_{x t}\right)$ is greater than -3 , the likely cause of the distortions and break population is tidal stripping. We estimate $r_{\text {distort }} \sim 30^{\prime}$ from Figures $18 \mathrm{~b}$ and 19 and $r_{\text {break }} \sim 60^{\prime}$ and $\gamma_{x t}=-2$ from Figure 16. These numbers satisfy the above conditions for tidal stripping set by the simulations.

Yet another explanation for the break population is that it is a dynamically hotter, but bound component to the Sculptor system. If Sculptor has a significant, extended dark matter component, as has recently been reported for the Draco dSph (Kleyna et al. 2002), a bound stellar component extending to twice the King limiting radius might be accommodated. However, Sculptor is at the low end of the dSph $M / L$ range, with an inferred central $M / L \sim 10 M_{\odot} / L_{\odot},{ }^{16}$ compared to the central Draco $M / L$ of $\sim 100 M_{\odot} / L_{\odot}$ (or even larger — $440 M_{\odot} / L_{\odot}$ — as recently reported by Kleyna et al. 2002), so it seems less likely that the break population of Sculptor stars is sheltered within a massive dark matter halo of the inferred size of Draco's. On the other hand, the extraordinary inflation of the Draco $M / L$ by Kleyna et al. (2003) was a direct result of an observed flat velocity dispersion, as we have seen here ${ }^{17}$. Given this tendency for some modelers to accommodate the flat dispersions profiles by adding mass to the dSph at large radii, it is likely that a convincing dismissal of the "large dark matter halo" interpretation will require tracking the break population, both photometrically and spectroscopically, to even larger radii, to the point where obvious tidal tails should be discernible. However, because N-body models of disrupting satellites have been found that can explain flat velocity dispersion profiles and $\mathrm{K}+\mathrm{B}$ radial density profiles

\footnotetext{
${ }^{16}$ Although we have redefined the structural parameters of the Sculptor system here, our core radius $(7 ! 14 \pm 0 ! 33)$ is only slightly larger than that found previously by IH95 $(5 ! 8 \pm 1.6)$ and values of our velocity dispersion at small radii are insignificantly different from that of QDP $\left(6.2 \pm 1.1 \mathrm{~km} \mathrm{~s}^{-1}\right.$; see Figure 12). Since $M / L \propto \sigma_{v}^{2} / r_{c}$, a recalculation of $M / L$ using our new values would yield a $15-20 \%$ smaller value than previously determined $(M / L=10.9$; IH95); an insignificant difference considering the large errors associated with the calculation.

${ }^{17}$ We note Wilkinson et al. (2004) have recently suggested a fall in the outer velocity dispersion for both Draco and Ursa Minor. However, our own work on Ursa Minor (Paper IX) is inconsistent with the presence of a such a "cold points" at large radii, and Łokas et al. (2004) have also raised concerns about the Wilkinson et al. (2004) cold population in the outer parts of Draco.
} 
(Mu05b), we believe that the weight of evidence for tidal disruption models is now greater, and so explore this interpretation further here.

Assuming the break population is from tidal disruption, we can calculate the fractional mass-loss rate of Sculptor based on our newly mapped radial profile. For satellites on circular orbits, the mass loss is due to constant stripping of the satellite over time. For more eccentric orbits, the mass loss comes in more violent pulses when the satellite passes perigalacticon. Johnston, Sigurdsson \& Hernquist (1999) give two prescriptions for the derivation of the mass-loss rate, which have been modified by the more recent analysis of Johnston et al. (2002). Using the corrected formula for the mass loss from the above simulations, Table 14 gives the resulting fractional mass-loss rates for the various samples in this paper. The last "reliable" point that defines $r_{\text {break }}$ is taken as the last point that is consistent with the $\Sigma \propto r^{-2}$ fall-off. The mean of the values in Table 14 are $(d f / d t)_{1}=0.042 \mathrm{Gyr}^{-1}$ and $(d f / d t)_{2}=0.083 \mathrm{Gyr}^{-1}$. This is a relatively mild fractional mass-loss rate yet places it squarely on the trend of increasing fractional mass-loss rate with inferred $(M / L)_{V \text {,tot noted }}$ by Majewski (2003, see also Majewski et al. 2002, 2005b). Majewski et al. (2005b) discuss as one explanation for this trend an increase in tidal disruption in systems that have a larger inferred $M / L$ (see also Majewski 2002). That Sgr — which is clearly losing mass into tidal arms to create its break population — participates in this trend lends support for this notion (Majewski 2003; Majewski et al. 2005b).

If Sculptor is losing stars due to tidal stripping, our analysis of the different stellar populations (\$5.4) suggests that Sculptor would be contributing stars to the MW halo predominantly from its more metal-poor population. Extrapolated back in time, this differential mass loss would suggest that Sculptor once had a larger metal-poor population. Whereas the two Sculptor populations - as we have divided them — are roughly equal in proportion, if we extrapolate the Sculptor mass loss backwards for 12 Gyr we find that the original metal poor population would have been about 50\% larger. A similar argument has been used to estimate the original balance of populations in the Carina dSph (Paper II; Majewski et al. 2002).

KBW would like to thank the Department of Astronomy at the University of Virginia for its active support of undergraduate research. Preliminary portions of this work were used for completion of a senior thesis at the University of Virginia. Both SRM and KBW thank his Ph.D. thesis adviser, M. A. Bershady, for patience while a large portion of this undergraduate project was completed by KBW at the University of Wisconsin - Madison. We acknowledge support from NSF grants AST-9702521, AST-0307851 and AST-0307417, a Cottrell Scholar Award from The Research Corporation, NASA/JPL contract 1228235, the David and Lucile Packard Foundation, and the generous support of The F. H. Levinson 
Fund of the Peninsula Community Foundation. PMF holds a Levinson Graduate Fellowship awarded through the Peninsula Community Foundation and is also supported by the Virginia

Space Grant Consortium. SRM is extremely grateful to the Carnegie Observatories for a Visiting Associateship, which allowed access to the Swope telescope to undertake this survey of the Sculptor dSph. Finally, we appreciate useful conversations with Ricardo Muñoz and Kathryn Johnston, and helpful comments from the referee that helped improve the discussion.

\section{REFERENCES}

Aaronson, M. 1983, ApJ, 266, L11

Aaronson, M., \& Olszewski, E. 1987, AJ, 94, 657

Aparicio, A., Carrera, R., \& Martínez-Delgado, D. 2001, AJ, 122, 2524

Armandroff, T., \& Da Costa, G. S. 1986, AJ, 92, 777

Bernstein, R., Shectman, S. A., Gunnels, S. M., Mochnacki, S., \& Athey, A. E. 2003, Proc. SPIE, 4841, 1694

Bessel, M. S. 2001, PASP, 113, 66

Bullock, J. S, Kravtsov, A. V., \& Weinberg, D. H. 2001, ApJ, 548, 33

Burkert, A. 1997, ApJ, 474, 99

Cudworth, K. M., \& Rees, R. F. 1991, PASP, 103, 470

Da Costa, G. S. 1984, ApJ, 285, 483

Da Costa, G. S., Armandroff, T. E., Caldwell, N., \& Seitzer, P. 1996, AJ, 112, 2576

Demers, S., Kunkel, W. E., \& Krauter, A. 1980, AJ, 85, 1587

Eskridge, P. B. 1988a, AJ, 95, 445

Eskridge, P. B. 1988b, AJ, 95, 1706

Eskridge, P. B. 1988c, AJ, 96, 1336

Faber, S. M., \& Lin, D. N. C. 1983, ApJ, 266, L17

Geisler, D. 1990, PASP, 102, 344 
Geisler, D. 1996, AJ, 111, 480

Goldsmith, C. G. 1993, in new Perspectives on Stellar Pulsation and Pulsating Variable Stars, eds. J. M. Nemec \& J. M. Matthews (Cambridge: Cambridge Univ. Press), 358

Gómez-Flechoso, M. A. 1998, Ap\&SS, 263, 155

Gould, A., Guhathakurta, P., Richstone, D., \& Flynn, C. 1992, ApJ, 388, 345

Grebel, E. K., Roberts, W. J., \& van de Rydt, F. 1994, in "The Local Group: Comparative and Global Properties, ESO Conference and Workshop Proceedings, Third CTIO/ESO Workshop on The Local Group", eds. A. Layden, C. Smith, \& J. Storm, (Garching bei München: ESO), 148

Grillmair, C. J., Freeman, K. C., Irwin, M., \& Quinn, P. J. 1995, AJ, 109, 2553

Harbeck, D., Grebel, E. K., Holtzman, J., Guhathakurta, P., Brandner, W., Geisler, D., Sarajedini, A., Dolphin, A., Hurley-Keller, D., \& Mateo, M. 2001, AJ, 122, 3092

Harding, P. Morrison, H. L., Olszewski, E. W., Arabadjis, J., Mateo, M., Dohm-Palmer, R. C., Freeman, K. C., and Norris. J. E. 2001, AJ, 122, 1397

Hargreaves, J. C., Gilmore, G., Irwin, M. J., \& Carter, D. 1994, MNRAS, 269, 957

Harris, W. E., Fitzgerald, M. P., \& Reed, B. C. 1981, PASP, 93, 507

Hayashi, E., Navarro, J. F., Taylor, J. E., Stadel, J., \& Quinn, T. 2003, ApJ, 584, 541

Hodge, P. W. 1961, AJ, 66, 384 (H61)

Hurley-Keller, D., Mateo, M., \& Grebel, E. K. 1999, ApJ, 523, L25

Ibata, R. A., Gilmore, G., \& Irwin, M. J. 1995, MNRAS, 277, 781

Ibata, R., Irwin, M., Lewis, G. F. \& Stolte, A. 2001, ApJ, 547, 133

Innanen, K. A., \& Papp, K. A. 1979, AJ, 84, 601

Irwin, M., \& Hatzidimitriou, D. 1995, MNRAS, 277, 1354 (IH95)

Johnston, K. V. 1998, ApJ, 495, 297

Johnston, K. V., Choi, P. I., \& Guhathakurta, P. 2002, AJ, 124, 127

Johnston, K. V., Sigurdsson, S., \& Hernquist, L. 1999, MNRAS, 302, 771 
Johnston,K. V., Spergel, D. N., \& Haydn, C. 2002, ApJ, 570, 656

Kaluzny, J., Kubiak, M., Szymański, M., Udalski, A., Krzemiński, W., \& Mateo, M. 1995 A\&AS, 112, 407

Kauffmann, G. \& White, S. D. M. 1993, MNRAS, 261, 921

King, I. R. 1962, AJ, 67, 471

King, I. R. 1966, AJ, 71, 64

Kleyna, J. T., Geller, M. J., Kenyon, S. J., Kurtz, M. J., \& Thorstensen, J. R. 1998, AJ, 115,2359

Kleyna, J., Geller, M., Kenyon, S. \& Kurtz, M. 1999, AJ, 117, 1275

Kleyna, J. T., Wilkinson, M. I., Evans, N. W., \& Gilmore, G. 2001, ApJ, 563, L115

Kleyna, J. T., Wilkinson, M. I., Evans, N. W., Gilmore, G. 2004, MNRAS, 354, 66

Kleyna, J., Wilkinson, M. I., Evans, N. W., Gilmore, G., \& Frayn, C. 2002, MNRAS, 330, 792

Kleyna, J. T., Wilkinson, M. I., Gilmore, G., \& Evans, N. W. 2003, ApJ, 588, L21

Kocevski, D. D., \& Kuhn, J. R. 2000, BAAS, 32, 1446

Kroupa, P. 1997, New Astronomy 2, 139

Kuhn, J. R., 1993, ApJ, 409, L13

Kuhn, J. R., Smith, H. A., \& Hawley, S. L. 1996, ApJ, 469, L93

Kunkel, W. E. \& Demers, S. 1977, ApJ, 214, 21

Lacey, C. \& Cole, S. 1993, MNRAS, 262, 627

Lejuene, T. \& Buser, R. 1996, Baltic Astronomy, 5, 399

Light, R. M. 1988, Ph.D. Dissertation, Yale University

Łokas, E., Mamon, G. \& Prada, F. 2004, MNRAS, submitted (astro-ph/0411694)

Majewski, S. R. 2002, in The Shapes of Galaxies and Their Dark Halos, Ed. P. Natarajan. Singapore: World Scientific, p.214 
Majewski, S. R., Kron, R. G., Koo, R. C., \& Bershady, M. A. 1994, PASP, 106, 1258

Majewski, S. R., Siegel, M. H., Patterson, R. J., \& Rood, R. T. 1999, ApJ, 520, L33

Majewski, S. R., Ostheimer, J. C., Patterson, R. J., Kunkel, W. E., Johnston, K. V., \& Geisler, D. 2000a, AJ, 119, 760 (Paper II)

Majewski, S. R., Ostheimer, J. C., Kunkel, W. E., \& Patterson, R. J. 2000b, AJ, 120, 2550 (Paper I)

Majewski, S. R., Patterson, R. J., Palma, C., Westfall, K. B., Siegel, M. H, Ostheimer, J. C., Frinchaboy, P. M., Link, R., Sohn, S., Kunkel, W. E., \& Johnston, K. V. 2002, in ASP Conf. Ser. 285, "Modes of Star Formation and the Origin of Field Populations", eds. E. K. Grebel \& W. Brandner, (San Francisco: ASP), 199

Majewski, S. R., Skrutskie, M. F., Weinberg, M. D., \& Ostheimer, J. C. 2003, ApJ, 599, 1082

Majewski, S. R. 2003, in ASP Conf. Ser. 296, "New Horizons in Globular Cluster Astronomy", eds. G. Djorgovski \& M. Riello, 447

Majewski et al. 2004a, AJ, 128, 245

Majewski et al. 2005a, AJ, in press (Paper VI)

Majewski et al. 2005b, in preparation

Martínez-Delgado, D., Alonso-García, J., Aparicio, A., \& Gómez-Flechoso, M. A. 2001, ApJ, 549, L63

Mateo, M. 1997, in ASP Conf. Ser. 116, "The Second Stromlo Symposium: The Nature of Elliptical Galaxies", eds. M. Arnaboldi, G. S. Da Costa, and P. Saha, 259

Mateo, M. 1998, ARA\&A, 36, 435

Mateo, M., Olszewski, E., Welch, D. L., Fischer, P., \& Kunkel, W. 1991, AJ, 102, 914

Mayer, L., Governato, F., Colpi, M., Moore, B., Quinn, T., Wadsley, J., Stadel, J., \& Lake, G. 2001, ApJ, 547, L123

Monet et al. 1998, The USNO-A2.0 Catalogue, (Washington D.C., U.S. Naval Observatory)

Morrison, H. L., Olszewski, E. W., Mateo, M., Norris, J. E., Harding, P., Dohm-Palmer, R. C., \& Freeman, K. C. 2001, AJ, 121, 283 
Muñoz, R. R., et al. 2005a, ApJ, submitted (Paper IX)

Muñoz, R. R., et al. 2004, in preparation (Mu05b)

Navarro, J. F., Frenk, C. S., \& White S. D. M. 1996, ApJ, 462, 563

Navarro, J. F., Frenk, C. S., \& White S. D. M. 1997, ApJ, 490, 493

Norris, J. \& Bessell, M. S. 1978, ApJ, 225, 49

Odenkirchen, M., et al. 2001, AJ, 122, 2538

Odenkirchen, M., et al. 2002, ApJ, 548, L165

Oh, K. S., Lin, D. N. C., \& Aarseth, S. J. 1995, ApJ, 442, 142

Ostheimer, J. C. 2002, Ph.D. Dissertation, University of Virginia

Ostheimer, J. C. 2005, in prep.

Palma, C., Majewski, S. R., Siegel, M. H., Patterson, R. J., Ostheimer, J. C., Link, R. 2003, AJ, 125, 1352 (Paper IV)

Paltoglou, G., \& Bell, R. A. 1994, MNRAS, 268, 793

Piatek, S. \& Pryor, C. 1995, AJ, 109, 1071

Piatek, S., Pryor, C., Armandroff, T. E., \& Olszewski, E. W. 2001, AJ, 121, 841

Piatek, S., Pryor, C., Armandroff, T. E., \& Olszewski, E. W. 2002, AJ, 123, 2511

Press, W. H. \& Schechter, P. 1974, ApJ, 187, 425

Press, W. H., Teukolsky, S. A., Vetterlin, W. T., \& Flannery, B. P. 2002, Numerical Recipes in $\mathrm{C}++$ (Cambridge: Cambridge Univ. Press), 628

Queloz, D., Dubath, P., \& Pasquini, L. A\&A, 300, 31 (QDP)

Reid, N. \& Majewski, S. R. 1993, ApJ, 409, 635

Rockosi, C. M. et al. 2002, AJ, 124, 349

Schlegel, D. J., Finkbeiner, D. P., \& Davis, M. 1998, ApJ, 500, 525

Schweitzer, A. E., Cudworth, K. M., Majewski, S. R., \& Suntzeff, N. B. 1995, AJ, 110, 2747

Shapley, H. 1938a, Harvard Bull., 908, 1 
Shapley, H. 1938b, Nature, 142, 715

Shapley, H. 1939, Proc. Natl. Acad. Sci. U. S., 25, 565

Shetrone, M., Venn, K. A., Tolstoy, E., Primas, F., Hill, V., \& Kaufer, A. 2003, AJ, 125, 684

Smith, G. H. \& Dopita, M. A. 1983, ApJ, 271, 113

Smith, H. A., Kuhn, J. R., \& Hawley, S. L. 1997, in ASP Conf. Ser. 127, "Proper Motions and Galactic Astronomy", ed. R. M. Humphreys (San Francisco: ASP), 163

Sohn, S., Majewski, S. R., Siegel, M. H., Ostheimer, J. C., Patterson, R. J., \& Muñoz, R. R. 2004, in preparation (Paper X)

Stetson, P. B. 1990, PASP, 102, 932

Stetson, P. B. 1992, in ASP Conf. Ser. 25, Astronomical Data Analysis Software and Systems, eds. D. M. Worrall, C. Biemesderfer, \& J. Barnes (San Francisco: ASP), 297

Thackeray, A. D. 1950, The Observatory, 70, 144

Tolstoy, E., Irwin, M. J., Cole, A. A., Pasquini, L., Gilmozzi, R., \& Gallagher, J. S. 2001, MNRAS, 327, 918

Tolstoy et al. 2004, ApJ, 617, L119

Tolstoy, E., Venn, K. A., Shetrone, M., Primas, F., Hill, V., Kaufer, A., \& Szeifert, T. 2003, AJ, 125,707

Tonry, J. \& Davis, M. 1979, AJ, 84, 1511

van Agt, S. L. Th. 1973, in IAU Colloq. 21, "Variable Stars in Globular Clusters and in Related Systems", eds. Fernie, J. D. (Dordrecht: Reidel), 35

Walcher, J., Fried, J. W., Burkert, A., \& Klessen, R. S. 2003, A\&A, 406, 847 (W03)

Westfall, K. B., Ostheimer, J. C., Frinchaboy, P. M., Patterson, R. J., Majewski, S. R., \& Kunkel, W. E. 2000, BAAS, 33, 718

Wilkinson, M. I., Kleyna, J. T., Evans, N. W., Gilmore, G. F., Irwin, M. J., \& Grebel, E. K. 2003, ApJ, 611, L21 
Table 1. Imaging Observations

\begin{tabular}{ccccc}
\hline \hline UT Date & Seeing(" ${ }^{\mathrm{a}}$ & Moon Illum. & $N_{\text {fields }}$ & $N_{\text {phot }}$ \\
\hline \multicolumn{5}{c}{ SITe \#1 Chip } \\
\hline 26 Aug - 02 Sep 1999 & 1.3 & $1.0-0.5$ & 16 & 7 \\
26 Oct - 30 Oct 1999 & 1.5 & $0.9-0.5$ & 11 & 6 \\
19 Dec - 24 Dec 1999 & 1.5 & $0.9-0.9$ & 14 & 4 \\
30 Oct - 08 Nov 2000 & 1.8 & $0.2-0.9$ & 18 & 0 \\
\hline \multicolumn{7}{c}{ SITe \#3 Chip } \\
\hline 23 Oct - 24 Oct 2001 & 1.6 & $0.5-0.6$ & \\
16 Nov - 20 Nov 2001 & 1.2 & $0.0-0.3$ & 33 & 33 \\
\hline
\end{tabular}

${ }^{a}$ All seeing values are averages over all Sculptor observations for each run. 
Table 2. Adopted Radial Velocities for Multiple Observations

\begin{tabular}{|c|c|c|c|c|c|}
\hline ID & $v_{\text {hel }^{\mathrm{a}}}^{\mathrm{a}}$ & $C C P$ & $Q$ & $s\left(v_{\text {hel }}\right)^{\mathrm{a}}$ & UT Date \\
\hline 1000427 & 111.68 & $\ldots$ & $\ldots$ & 4.82 & $\ldots$ \\
\hline$\ldots$ & 113.10 & 1.00 & 7 & $\ldots$ & 13 Nov 2000 \\
\hline$\ldots$ & 103.50 & 0.25 & 4 & $\ldots$ & 07Oct2001 \\
\hline 1000834 & 103.62 & $\ldots$ & $\ldots$ & 0.95 & $\ldots$ \\
\hline$\cdots$ & 101.10 & 0.73 & 7 & $\cdots$ & 13 Nov 2000 \\
\hline$\ldots$ & 103.80 & 1.02 & 7 & $\ldots$ & 07Oct2001 \\
\hline 1000844 & 126.64 & $\ldots$ & $\ldots$ & 14.04 & $\ldots$ \\
\hline$\ldots$ & 110.10 & 1.32 & 7 & $\ldots$ & 13 Nov2000 \\
\hline$\ldots$ & 132.60 & 0.62 & 7 & $\cdots$ & 07 Oct2001 \\
\hline 1002429 & 106.33 & $\ldots$ & $\ldots$ & 1.51 & $\ldots$ \\
\hline$\ldots$ & 109.00 & 1.06 & 7 & $\ldots$ & 13 Nov2000 \\
\hline$\cdots$ & 105.90 & 1.31 & 7 & $\ldots$ & 07Oct2001 \\
\hline 1003967 & 98.27 & $\ldots$ & $\ldots$ & 5.56 & $\ldots$ \\
\hline$\ldots$ & 108.10 & 0.96 & 7 & $\ldots$ & 13 Nov 2000 \\
\hline$\ldots$ & 96.70 & 0.69 & 7 & $\ldots$ & 07Oct2001 \\
\hline 1005457 & 111.96 & $\ldots$ & $\ldots$ & 5.99 & $\ldots$ \\
\hline$\ldots$ & 104.90 & 1.16 & 7 & $\ldots$ & 13 Nov 2000 \\
\hline$\ldots$ & 114.50 & 0.97 & 7 & $\ldots$ & 07Oct2001 \\
\hline 1007204 & 107.22 & $\ldots$ & $\ldots$ & 0.78 & $\ldots$ \\
\hline$\ldots$ & 108.60 & 0.62 & 7 & $\ldots$ & 13 Nov 2000 \\
\hline$\cdots$ & 107.00 & 0.70 & 7 & $\ldots$ & 07Oct2001 \\
\hline 1008239 & 110.83 & $\ldots$ & $\ldots$ & 11.33 & $\ldots$ \\
\hline$\ldots$ & 113.50 & 1.12 & 7 & $\ldots$ & 07Oct2001 \\
\hline$\ldots$ & 86.80 & 0.36 & 7 & $\ldots$ & 07Oct2001 \\
\hline 1013815 & 101.69 & $\ldots$ & $\ldots$ & 3.99 & $\ldots$ \\
\hline$\ldots$ & 106.40 & 1.14 & 7 & $\ldots$ & 13 Nov 2000 \\
\hline$\ldots$ & 100.00 & 0.83 & 7 & $\ldots$ & 07Oct2001 \\
\hline 1013921 & 110.00 & . & $\cdots$ & 0.00 & $\ldots$ \\
\hline$\ldots$ & 110.00 & 0.88 & 7 & $\ldots$ & 13 Nov 2000 \\
\hline$\ldots$ & 110.00 & 0.56 & 7 & $\ldots$ & 07Oct2001 \\
\hline 1015830 & 107.88 & $\ldots$ & $\cdots$ & 3.95 & $\ldots$ \\
\hline$\cdots$ & 100.90 & 0.88 & 7 & $\ldots$ & 13 Nov 2000 \\
\hline$\ldots$ & 109.00 & 0.63 & 7 & $\ldots$ & 07Oct2001 \\
\hline 1017220 & 102.57 & $\ldots$ & $\ldots$ & 6.30 & $\ldots$ \\
\hline$\cdots$ & 110.00 & 1.05 & 7 & $\ldots$ & 13 Nov 2000 \\
\hline$\ldots$ & 99.90 & 0.84 & 7 & $\ldots$ & 07Oct2001 \\
\hline 1017304 & 112.02 & $\ldots$ & $\ldots$ & 4.06 & $\ldots$ \\
\hline
\end{tabular}


Table 2-Continued

\begin{tabular}{|c|c|c|c|c|c|}
\hline ID & $v_{\text {hel }^{\mathrm{a}}}$ & $C C P$ & $Q$ & $s\left(v_{\text {hel }}\right)^{\mathrm{a}}$ & UT Date \\
\hline$\ldots$ & 111.30 & 0.51 & 6 & $\ldots$ & 07Oct2001 \\
\hline$\ldots$ & 123.50 & 0.27 & 5 & $\ldots$ & 07Oct2001 \\
\hline 1017546 & 110.44 & $\ldots$ & $\ldots$ & 4.05 & $\ldots$ \\
\hline$\ldots$ & 99.70 & 0.81 & 7 & $\ldots$ & 13 Nov2000 \\
\hline$\ldots$ & 111.20 & 1.25 & 7 & $\ldots$ & 07 Oct 2001 \\
\hline 1017864 & 101.53 & $\ldots$ & $\ldots$ & 9.73 & $\ldots$ \\
\hline$\ldots$ & 113.00 & 1.33 & 7 & $\ldots$ & 13 Nov2000 \\
\hline$\ldots$ & 97.40 & 0.57 & 7 & $\ldots$ & 07Oct2001 \\
\hline 1019945 & 114.07 & $\ldots$ & $\ldots$ & 7.90 & $\ldots$ \\
\hline$\ldots$ & 100.10 & 0.80 & 7 & $\ldots$ & 13 Nov 2000 \\
\hline$\ldots$ & 116.30 & 0.79 & 7 & $\ldots$ & 07Oct2001 \\
\hline 1020962 & 114.31 & $\ldots$ & $\ldots$ & 2.50 & $\ldots$ \\
\hline$\ldots$ & 114.90 & 1.05 & 7 & $\ldots$ & 07Oct2001 \\
\hline$\ldots$ & 109.00 & 0.42 & 6 & $\ldots$ & 07 Oct 2001 \\
\hline 1021357 & 109.51 & $\ldots$ & $\ldots$ & 8.05 & $\ldots$ \\
\hline$\ldots$ & 119.00 & 1.26 & 7 & $\ldots$ & 13 Nov 2000 \\
\hline$\ldots$ & 106.10 & 0.80 & 7 & $\ldots$ & 07Oct2001 \\
\hline 2007352 & 117.64 & $\ldots$ & $\ldots$ & 2.24 & $\ldots$ \\
\hline$\ldots$ & 109.20 & 0.38 & 6 & $\ldots$ & 07Oct2001 \\
\hline$\ldots$ & 117.94 & 0.91 & 7 & $\ldots$ & 28Jan2004 \\
\hline 3005776 & 105.35 & $\ldots$ & $\cdots$ & 2.21 & $\ldots$ \\
\hline$\ldots$ & 107.30 & 0.55 & 7 & $\ldots$ & 13 Nov 2000 \\
\hline$\ldots$ & 104.10 & 0.38 & 6 & $\ldots$ & 07Oct 2001 \\
\hline 3010660 & 102.56 & $\ldots$ & $\ldots$ & 0.20 & $\ldots$ \\
\hline$\ldots$ & 102.60 & 0.95 & 7 & $\ldots$ & 07Oct2001 \\
\hline$\ldots$ & 102.00 & 0.25 & 4 & $\ldots$ & 07Oct2001 \\
\hline 3012313 & 90.79 & $\ldots$ & $\ldots$ & 8.11 & $\ldots$ \\
\hline$\ldots$ & 102.70 & 1.16 & 7 & $\ldots$ & 13 Nov 2000 \\
\hline$\ldots$ & 93.30 & 0.68 & 7 & $\ldots$ & 07Oct2001 \\
\hline$\ldots$ & 84.00 & 0.91 & 7 & $\ldots$ & 07Oct2001 \\
\hline 5001661 & 113.32 & $\ldots$ & .. & 0.61 & $\ldots$ \\
\hline$\ldots$ & 113.70 & 1.11 & 7 & $\ldots$ & 07Oct2001 \\
\hline$\ldots$ & 112.83 & 0.43 & 4 & $\ldots$ & 28Jan2004 \\
\hline 7015937 & 71.58 & $\ldots$ & $\cdots$ & 47.09 & $\ldots$ \\
\hline$\cdots$ & 107.10 & 1.46 & 7 & $\ldots$ & 07Oct2001 \\
\hline$\ldots$ & 40.36 & 0.61 & 7 & $\ldots$ & 31Dec2004 \\
\hline 28000278 & 107.18 & $\ldots$ & $\ldots$ & 11.36 & $\ldots$ \\
\hline
\end{tabular}


Table 2-Continued

\begin{tabular}{|c|c|c|c|c|c|}
\hline ID & $v_{\text {hel }^{\mathrm{a}}}$ & $C C P$ & $Q$ & $s\left(v_{\text {hel }}\right)^{\mathrm{a}}$ & UT Date \\
\hline$\ldots$ & 93.80 & 1.17 & 7 & $\ldots$ & 13 Nov 2000 \\
\hline$\ldots$ & 112.00 & 0.98 & 7 & $\ldots$ & 07 Oct 2001 \\
\hline 28000376 & 108.37 & $\ldots$ & $\ldots$ & 16.10 & $\ldots$ \\
\hline$\ldots$ & 89.40 & 1.08 & 7 & $\ldots$ & 13 Nov2000 \\
\hline$\ldots$ & 115.20 & 0.88 & 7 & $\ldots$ & 07 Oct 2001 \\
\hline 31000176 & 110.24 & $\ldots$ & $\ldots$ & 1.13 & $\ldots$ \\
\hline$\ldots$ & 103.80 & 1.15 & 7 & $\ldots$ & 13 Nov 2000 \\
\hline$\ldots$ & 111.30 & 0.78 & 7 & $\ldots$ & 07 Oct2001 \\
\hline$\ldots$ & 111.33 & 0.51 & 5 & $\ldots$ & 28Jan2004 \\
\hline$\ldots$ & 109.33 & 0.75 & 7 & $\ldots$ & 28Jan2004 \\
\hline 31000255 & 110.18 & $\ldots$ & $\ldots$ & 3.49 & $\ldots$ \\
\hline$\ldots$ & 89.40 & 0.83 & 7 & $\ldots$ & 13 Nov2000 \\
\hline$\ldots$ & 109.00 & 1.13 & 7 & $\ldots$ & 07 Oct2001 \\
\hline$\ldots$ & 111.02 & 0.73 & 6 & $\ldots$ & 28Jan2004 \\
\hline 31000262 & 80.91 & $\ldots$ & $\ldots$ & 54.22 & $\ldots$ \\
\hline$\ldots$ & 90.40 & 0.33 & 5 & $\ldots$ & 13 Nov 2000 \\
\hline$\ldots$ & 9.60 & 0.54 & 7 & $\ldots$ & 07 Oct2001 \\
\hline$\ldots$ & 108.62 & 0.95 & 4 & $\ldots$ & 30Dec2004 \\
\hline 31000458 & 106.80 & $\ldots$ & $\ldots$ & 4.24 & $\ldots$ \\
\hline$\ldots$ & 99.30 & 1.26 & 7 & $\ldots$ & 13 Nov 2000 \\
\hline$\ldots$ & 108.00 & 1.04 & 7 & $\ldots$ & 07 Oct2001 \\
\hline 31000522 & 115.99 & $\ldots$ & $\ldots$ & 9.45 & $\ldots$ \\
\hline$\ldots$ & 166.10 & 0.34 & 4 & $\ldots$ & 13 Nov 2000 \\
\hline$\ldots$ & 115.10 & 1.11 & 7 & $\ldots$ & 07 Oct2001 \\
\hline 31000535 & 121.09 & $\ldots$ & $\ldots$ & 1.36 & $\ldots$ \\
\hline$\ldots$ & 165.40 & 0.20 & 4 & $\ldots$ & 13 Nov2000 \\
\hline$\ldots$ & 120.00 & 0.95 & 7 & $\ldots$ & 07 Oct 2001 \\
\hline$\ldots$ & 121.13 & 1.03 & 7 & $\ldots$ & 28Jan2004 \\
\hline 31000550 & 99.68 & $\ldots$ & $\ldots$ & 0.14 & $\ldots$ \\
\hline$\ldots$ & 99.60 & 1.16 & 7 & $\ldots$ & 13 Nov 2000 \\
\hline$\ldots$ & 99.80 & 0.37 & 6 & $\ldots$ & 07 Oct2001 \\
\hline 31000589 & 113.78 & $\ldots$ & $\ldots$ & 3.24 & $\ldots$ \\
\hline$\ldots$ & 117.60 & 1.05 & 7 & $\ldots$ & 13 Nov 2000 \\
\hline$\ldots$ & 112.40 & 0.52 & 7 & $\ldots$ & 07 Oct 2001 \\
\hline 31000811 & 3.58 & $\ldots$ & $\ldots$ & 27.08 & $\ldots$ \\
\hline$\ldots$ & 107.40 & 0.68 & 7 & $\ldots$ & 13 Nov 2000 \\
\hline$\ldots$ & -4.50 & 0.64 & 7 & $\ldots$ & 07 Oct2001 \\
\hline
\end{tabular}


Table 2-Continued

\begin{tabular}{crcccc}
\hline \hline ID & $v_{\text {hel }^{\mathrm{a}}}$ & $C C P$ & $Q$ & $s\left(v_{\text {hel }}\right)^{\mathrm{a}}$ & UT Date \\
\hline$\ldots$ & 0.25 & 0.51 & 4 & $\ldots$ & 30 Dec2004 \\
31000822 & $\mathbf{1 0 8 . 3 1}$ & $\cdots$ & $\cdots$ & 2.61 & $\cdots$ \\
$\ldots$ & 101.40 & 0.75 & 7 & $\ldots$ & 13 Nov2000 \\
$\ldots$ & 108.80 & 1.07 & 7 & $\cdots$ & 07 Oct2001 \\
\hline
\end{tabular}

${ }^{\mathrm{a}}$ Units are $\mathrm{km} \mathrm{s}^{-1}$. 
Table 3. Comparison with Literature Radial Velocities

\begin{tabular}{|c|c|c|c|c|c|}
\hline ID & $v_{\text {hel }^{\mathrm{a}}}$ & $\epsilon_{v_{\text {hel }}}^{\mathrm{a}, \mathrm{b}}$ & $C C P$ & $Q$ & Reference $^{c}$ \\
\hline 1002429 & 106.33 & 3.71 & $\ldots$ & 8 & 0 \\
\hline H185 & 125.50 & 4.70 & $\ldots$ & $\ldots$ & 1 \\
\hline H185 & 110.00 & 1.80 & $\cdots$ & $\cdots$ & 2 \\
\hline 1003537 & 117.50 & 4.00 & 0.69 & 7 & 0 \\
\hline H241 & 116.10 & 4.70 & $\cdots$ & $\ldots$ & 1 \\
\hline H241 & 119.70 & 2.10 & $\cdots$ & $\ldots$ & 2 \\
\hline 1014728 & 99.20 & 4.00 & 0.42 & 6 & 0 \\
\hline H394 & 109.70 & 3.30 & $\ldots$ & $\ldots$ & 2 \\
\hline 1015134 & 91.50 & 4.00 & 0.56 & 7 & 0 \\
\hline H499 & 104.70 & 3.30 & $\cdots$ & $\cdots$ & 1 \\
\hline H499 & 105.50 & 1.70 & $\cdots$ & $\cdots$ & 2 \\
\hline 1015830 & 107.88 & 3.71 & $\ldots$ & 8 & 0 \\
\hline H97 & 115.70 & 3.30 & $\ldots$ & $\ldots$ & 1 \\
\hline H97 & 113.70 & 1.50 & $\cdots$ & $\cdots$ & 2 \\
\hline 1017220 & 102.57 & 3.71 & $\ldots$ & 8 & 0 \\
\hline K328 & 102.80 & 3.30 & $\cdots$ & $\ldots$ & 2 \\
\hline 1019417 & 99.00 & 4.00 & 0.97 & 7 & 0 \\
\hline H511 & 103.40 & 2.30 & $\cdots$ & $\cdots$ & 1 \\
\hline H511 & 101.30 & 1.50 & $\ldots$ & $\cdots$ & 2 \\
\hline 1020962 & 114.31 & 2.83 & $\ldots$ & 8 & 0 \\
\hline H372 & 105.80 & 4.70 & $\cdots$ & $\cdots$ & 1 \\
\hline H372 & 116.60 & 1.60 & $\cdots$ & $\cdots$ & 2 \\
\hline 1021965 & 108.20 & 4.00 & 1.14 & 7 & 0 \\
\hline H376 & 96.90 & 4.70 & $\ldots$ & $\ldots$ & 1 \\
\hline H376 & 98.60 & 1.70 & $\cdots$ & $\cdots$ & 2 \\
\hline 3005776 & 105.35 & 3.71 & $\ldots$ & 8 & 0 \\
\hline H144 & 99.10 & 4.70 & $\ldots$ & $\ldots$ & 1 \\
\hline H144 & 100.40 & 1.80 & $\ldots$ & $\ldots$ & 2 \\
\hline
\end{tabular}

${ }^{\mathrm{a}}$ Units are $\mathrm{km} \mathrm{s}^{-1}$.

${ }^{\mathrm{b}}$ For the current study, velocity errors provided here are either those adopted for the specific run or, when multi- 
ple observations were available, the error on the weighted mean velocity.

${ }^{\mathrm{c}}$ References: (0) This paper; (1) Armandroff \& Da Costa (1986); (2) QDP. 
Table 4. Heliocentric Radial Velocities of Selected Stars

\begin{tabular}{|c|c|c|c|c|c|c|c|c|c|c|c|c|}
\hline ID & $\alpha(\mathrm{J} 2000.0)$ & $\delta(\mathrm{J} 2000.0)$ & UT Date ${ }^{a}$ & $M$ & $M-T_{2}$ & $M-D$ & $v_{\text {hel }} \mathrm{b}$ & $\epsilon_{v_{\text {hel }}{ }^{c}}$ & $C C P$ & $Q$ & $a\left({ }^{\prime}\right)$ & $\mathrm{P} / \mathrm{V}^{\mathrm{d}}$ \\
\hline 1000425 & 005909.40 & -335054.5 & 13Nov2000 & $18.39 \pm 0.03$ & $1.54 \pm 0.04$ & $0.02 \pm 0.04$ & 99.50 & $\ldots$ & 0.93 & 7 & 17.0031 & $\mathrm{Y} / \mathrm{Y}$ \\
\hline 1000427 & 005911.70 & $-33 \quad 3049.8$ & $\ldots$ & $18.34 \pm 0.02$ & $1.37 \pm 0.03$ & $0.03 \pm 0.03$ & 111.68 & 3.71 & $\ldots$ & 8 & 19.7936 & $\mathrm{Y} / \mathrm{Y}$ \\
\hline 1000474 & 005910.24 & -334553.3 & 07 Oct 2001 & $19.38 \pm 0.08$ & $1.15 \pm 0.09$ & $0.07 \pm 0.10$ & 106.00 & & 1.00 & 7 & 12.6904 & $\mathrm{Y} / \mathrm{Y}$ \\
\hline 1000834 & 005912.08 & -334620.9 & $\ldots$ & $17.53 \pm 0.02$ & $1.59 \pm 0.03$ & $0.01 \pm 0.03$ & 103.62 & 3.71 & $\ldots$ & 8 & 12.6169 & $\mathrm{Y} / \mathrm{Y}$ \\
\hline 1000844 & $00 \quad 5912.71$ & $\begin{array}{lll}-33 & 41 & 09.3\end{array}$ & $\ldots$ & $17.96 \pm 0.02$ & $1.46 \pm 0.03$ & $-0.00 \pm 0.03$ & 126.64 & 3.71 & $\ldots$ & 8 & 11.2082 & $\mathrm{Y} / \mathrm{Y}$ \\
\hline 1001140 & 005914.55 & $-3340 \quad 39.8$ & 13 Nov2000 & $17.70 \pm 0.02$ & $1.72 \pm 0.03$ & $-0.03 \pm 0.03$ & 100.10 & $\ldots$ & 0.93 & 7 & 10.9588 & $\mathrm{Y} / \mathrm{Y}$ \\
\hline 1001378 & 005915.76 & -334248.5 & 13 Nov 2000 & $17.64 \pm 0.02$ & $1.72 \pm 0.03$ & $-0.01 \pm 0.03$ & 109.70 & $\ldots$ & 0.86 & 7 & 10.4815 & $\mathrm{Y} / \mathrm{Y}$ \\
\hline 1001547 & 005916.93 & $-3340 \quad 10.5$ & 13 Nov2000 & $17.30 \pm 0.02$ & $1.83 \pm 0.02$ & $-0.05 \pm 0.03$ & 100.60 & $\ldots$ & 1.11 & 7 & 10.6561 & $\mathrm{Y} / \mathrm{Y}$ \\
\hline 1001825 & $0059 \quad 17.77$ & $\begin{array}{lll}-33 & 46 & 01.7\end{array}$ & 13 Nov2000 & $17.66 \pm 0.02$ & $1.68 \pm 0.03$ & $0.03 \pm 0.03$ & 115.70 & $\cdots$ & 1.12 & 7 & 11.3318 & $\mathrm{Y} / \mathrm{Y}$ \\
\hline 1001924 & 005919.16 & $-33 \quad 3850.8$ & 07 Oct2001 & $17.70 \pm 0.02$ & $1.60 \pm 0.03$ & $0.03 \pm 0.03$ & 108.40 & $\ldots$ & 0.78 & 7 & 10.9324 & $\mathrm{Y} / \mathrm{Y}$ \\
\hline 1002353 & 005920.80 & -334404.8 & 13 Nov 2000 & $17.42 \pm 0.02$ & $1.64 \pm 0.03$ & $-0.00 \pm 0.03$ & 110.20 & $\ldots$ & 1.11 & 7 & 9.7262 & $\mathrm{Y} / \mathrm{Y}$ \\
\hline 1002429 & 005920.64 & -334856.6 & $\ldots$ & $17.41 \pm 0.02$ & $1.82 \pm 0.03$ & $-0.00 \pm 0.03$ & 106.33 & 3.71 & $\ldots$ & 8 & 13.3011 & $\mathrm{Y} / \mathrm{Y}$ \\
\hline 1003397 & $00 \quad 5926.25$ & $\begin{array}{lll}-33 & 46 & 52.8\end{array}$ & 13Nov 2000 & $17.35 \pm 0.02$ & $1.71 \pm 0.04$ & $0.00 \pm 0.03$ & 107.60 & $\ldots$ & 0.50 & 6 & 10.4524 & $\mathrm{Y} / \mathrm{Y}$ \\
\hline 1003537 & 005927.69 & $-3340 \quad 35.4$ & 07 Oct2001 & $17.38 \pm 0.02$ & $1.69 \pm 0.04$ & $0.03 \pm 0.03$ & 117.50 & $\ldots$ & 0.69 & 7 & 8.3723 & $\mathrm{Y} / \mathrm{Y}$ \\
\hline 1003694 & 005928.29 & $\begin{array}{lll}-33 & 42 & 07.2\end{array}$ & 13 Nov2000 & $17.30 \pm 0.02$ & $1.93 \pm 0.03$ & $-0.01 \pm 0.02$ & 132.70 & $\cdots$ & 1.07 & 7 & 7.8570 & $\mathrm{Y} / \mathrm{Y}$ \\
\hline 1003967 & 005930.44 & -333604.9 & $\ldots$ & $18.11 \pm 0.02$ & $1.41 \pm 0.03$ & $0.01 \pm 0.03$ & 98.27 & 3.71 & $\ldots$ & 8 & 11.6303 & $\mathrm{Y} / \mathrm{Y}$ \\
\hline 1004879 & 005933.99 & -334620.9 & 13 Nov 2000 & $17.67 \pm 0.02$ & $1.64 \pm 0.03$ & $0.00 \pm 0.03$ & 94.20 & $\cdots$ & 0.18 & 5 & 8.6774 & $\mathrm{Y} / \mathrm{Y}$ \\
\hline 1005103 & 005935.37 & $\begin{array}{lll}-33 & 44 & 09.2\end{array}$ & $\ldots$ & $17.58 \pm 0.02$ & $1.94 \pm 0.03$ & $-0.01 \pm 0.03$ & 110.93 & 1.68 & $\cdots$ & $\ldots$ & 6.8222 & $\mathrm{Y} / \mathrm{Y}$ \\
\hline 1005329 & 005937.23 & -333710.4 & 07 Oct 2001 & $17.84 \pm 0.02$ & $1.70 \pm 0.03$ & $0.01 \pm 0.03$ & 106.60 & $\ldots$ & 0.51 & 7 & 9.5869 & $\mathrm{Y} / \mathrm{Y}$ \\
\hline 1005457 & 005938.12 & -333507.9 & $\ldots$ & $17.75 \pm 0.02$ & $1.62 \pm 0.02$ & $0.03 \pm 0.02$ & 111.96 & 3.71 & $\ldots$ & 8 & 11.9028 & $\mathrm{Y} / \mathrm{Y}$ \\
\hline 1006178 & 005941.40 & -333846.9 & 07 Oct2001 & $17.86 \pm 0.02$ & $1.66 \pm 0.03$ & $0.01 \pm 0.03$ & 112.20 & $\cdots$ & 0.34 & 5 & 7.3290 & $\mathrm{Y} / \mathrm{Y}$ \\
\hline 1007204 & $00 \quad 5947.21$ & -333336.9 & $\ldots$ & $17.78 \pm 0.01$ & $1.52 \pm 0.02$ & $0.04 \pm 0.02$ & 107.22 & 3.71 & $\ldots$ & 8 & 13.2228 & $\mathrm{Y} / \mathrm{Y}$ \\
\hline 1007206 & 005946.42 & $\begin{array}{lll}-33 & 41 & 23.2\end{array}$ & 07 Oct 2001 & $17.56 \pm 0.02$ & $1.89 \pm 0.03$ & $-0.03 \pm 0.03$ & 107.10 & $\ldots$ & 1.10 & 7 & 4.3771 & $\mathrm{Y} / \mathrm{Y}$ \\
\hline 1007312 & 005946.16 & -334838.9 & 07Oct2001 & $17.82 \pm 0.02$ & $1.58 \pm 0.03$ & $0.02 \pm 0.03$ & 104.30 & $\cdots$ & 1.04 & 7 & 9.6380 & $\mathrm{Y} / \mathrm{Y}$ \\
\hline 1007362 & 005947.05 & -334254.0 & $\ldots$ & $18.05 \pm 0.03$ & $1.55 \pm 0.03$ & $-0.10 \pm 0.03$ & 118.00 & 3.30 & $\cdots$ & $\cdots$ & 3.9794 & $\mathrm{Y} / \mathrm{Y}$ \\
\hline 1007490 & 005947.26 & $\begin{array}{lll}-33 & 46 & 30.3\end{array}$ & $\ldots$ & $17.74 \pm 0.02$ & $1.71 \pm 0.03$ & $-0.01 \pm 0.03$ & 106.30 & 2.80 & $\ldots$ & $\ldots$ & 6.8700 & $\mathrm{Y} / \mathrm{Y}$ \\
\hline 1008239 & 005950.00 & -335348.4 & $\ldots$ & $18.34 \pm 0.02$ & $1.36 \pm 0.03$ & $0.01 \pm 0.03$ & 110.83 & 2.83 & $\cdots$ & 8 & 16.3321 & $\mathrm{Y} / \mathrm{Y}$ \\
\hline 1008522 & 005952.28 & -334454.5 & $\ldots$ & $17.44 \pm 0.03$ & $1.73 \pm 0.03$ & $0.01 \pm 0.03$ & 114.10 & 3.00 & $\cdots$ & 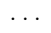 & 4.3948 & $\mathrm{Y} / \mathrm{Y}$ \\
\hline 1008996 & 005954.65 & -334342.4 & 13 Nov2000 & $17.35 \pm 0.02$ & $2.06 \pm 0.03$ & $-0.01 \pm 0.03$ & 106.50 & & 1.17 & 7 & 2.8601 & $\mathrm{~N}^{\mathrm{e}} / \mathrm{Y}$ \\
\hline 1010231 & $\begin{array}{lll}01 & 00 & 00.24\end{array}$ & -334447.6 & 07Oct2001 & $17.76 \pm 0.03$ & $1.58 \pm 0.04$ & $0.05 \pm 0.03$ & 116.50 & $\cdots$ & 0.73 & 7 & 3.3255 & $\mathrm{Y} / \mathrm{Y}$ \\
\hline 1010398 & $\begin{array}{lll}01 & 00 & 00.49\end{array}$ & -334935.4 & 07Oct2001 & $17.68 \pm 0.02$ & $1.48 \pm 0.03$ & $0.04 \pm 0.03$ & 106.90 & $\ldots$ & 0.27 & 5 & 9.9710 & $\mathrm{Y} / \mathrm{Y}$ \\
\hline 1010633 & $\begin{array}{lll}01 & 00 & 01.45\end{array}$ & $\begin{array}{lll}-33 & 51 & 16.4\end{array}$ & 07Oct 2001 & $17.94 \pm 0.03$ & $1.38 \pm 0.03$ & $0.03 \pm 0.03$ & 98.40 & $\ldots$ & 0.47 & 6 & 12.3217 & $\mathrm{Y} / \mathrm{Y}$ \\
\hline 1011098 & $0100 \quad 04.62$ & $\begin{array}{lll}-33 & 41 & 11.8\end{array}$ & $\ldots$ & $17.69 \pm 0.03$ & $1.53 \pm 0.04$ & $0.02 \pm 0.03$ & 77.90 & 3.80 & $\cdots$ & $\ldots$ & 2.0635 & $\mathrm{Y} / \mathrm{N}$ \\
\hline 1011494 & $\begin{array}{llll}01 & 00 & 06.74\end{array}$ & $\begin{array}{lll}-33 & 39 & 26.1\end{array}$ & $\ldots$ & $18.90 \pm 0.04$ & $1.26 \pm 0.04$ & $0.04 \pm 0.05$ & 107.37 & 1.51 & $\ldots$ & $\ldots$ & 4.5672 & $\mathrm{Y} / \mathrm{Y}$ \\
\hline 1011626 & $\begin{array}{llll}01 & 00 & 07.57\end{array}$ & $\begin{array}{lll}-33 & 37 & 03.7\end{array}$ & 07 Oct 2001 & $17.79 \pm 0.02$ & $1.63 \pm 0.03$ & $-0.01 \pm 0.03$ & 102.30 & $\cdots$ & 0.40 & 5 & 7.9460 & $\mathrm{Y} / \mathrm{Y}$ \\
\hline 1011721 & 010006.96 & $\begin{array}{lll}-33 & 47 & 09.2\end{array}$ & 07 Oct 2001 & $18.04 \pm 0.03$ & $1.47 \pm 0.04$ & $0.04 \pm 0.04$ & 110.80 & $\cdots$ & 0.51 & 5 & 6.3896 & $\mathrm{Y} / \mathrm{Y}$ \\
\hline 1011802 & $\begin{array}{llll}01 & 00 & 07.40\end{array}$ & $-3345 \quad 57.2$ & $\ldots$ & $17.80 \pm 0.03$ & $1.55 \pm 0.04$ & $0.03 \pm 0.04$ & 103.00 & 3.50 & $\cdots$ & $\cdots$ & 4.6874 & $\mathrm{Y} / \mathrm{Y}$ \\
\hline 1012230 & $\begin{array}{lll}01 & 00 & 08.98\end{array}$ & -335022.6 & 07 Oct 2001 & $17.70 \pm 0.02$ & $1.52 \pm 0.04$ & $-0.00 \pm 0.03$ & 126.40 & $\ldots$ & 0.70 & 7 & 10.9573 & $\mathrm{Y} / \mathrm{Y}$ \\
\hline 1012628 & 010012.13 & -333725.9 & 07 Oct2001 & $17.48 \pm 0.02$ & $1.87 \pm 0.03$ & $-0.01 \pm 0.02$ & 107.20 & $\cdots$ & 1.16 & 7 & 7.5696 & $\mathrm{Y} / \mathrm{Y}$ \\
\hline 1013365 & $\begin{array}{lll}01 & 00 & 14.82\end{array}$ & -334421.9 & $\cdots$ & $17.59 \pm 0.03$ & $1.62 \pm 0.04$ & $0.02 \pm 0.03$ & 112.40 & 3.40 & $\cdots$ & $\cdots$ & 2.9853 & $\mathrm{~N}^{\mathrm{f}^{\prime}} / \mathrm{Y}$ \\
\hline 1013815 & $\begin{array}{lll}01 & 00 & 18.20\end{array}$ & -333140.4 & $\cdots$ & $17.61 \pm 0.02$ & $1.84 \pm 0.02$ & $0.04 \pm 0.02$ & 101.69 & 3.71 & $\cdots$ & 8 & 15.8956 & $\mathrm{Y} / \mathrm{Y}$ \\
\hline 1013921 & $\begin{array}{llll}01 & 00 & 18.57\end{array}$ & $-33 \quad 3213.5$ & $\cdots$ & $17.92 \pm 0.01$ & $1.64 \pm 0.02$ & $0.05 \pm 0.02$ & 110.00 & 3.71 & $\cdots$ & 8 & 15.1407 & $\mathrm{Y} / \mathrm{Y}$ \\
\hline 1014064 & $\begin{array}{llll}01 & 00 & 18.29\end{array}$ & $-33 \quad 42 \quad 12.1$ & $\cdots$ & $17.74 \pm 0.03$ & $1.61 \pm 0.04$ & $-0.00 \pm 0.04$ & 108.60 & 3.90 & $\ldots$ & $\cdots$ & 2.6688 & $\mathrm{~N}^{\mathrm{f}^{\prime}} / \mathrm{Y}$ \\
\hline 1014331 & $\begin{array}{lll}01 & 00 & 18.31\end{array}$ & -335331.4 & 07 Oct2001 & $17.57 \pm 0.02$ & $1.72 \pm 0.03$ & $0.01 \pm 0.02$ & 123.60 & $\ldots$ & 1.00 & 7 & 15.5244 & $\mathrm{Y} / \mathrm{Y}$ \\
\hline 1014370 & $\begin{array}{lll}01 & 00 & 20.29\end{array}$ & -333534.4 & 13 Nov2000 & $17.68 \pm 0.02$ & $1.57 \pm 0.02$ & $0.01 \pm 0.02$ & 115.80 & $\ldots$ & 1.34 & 7 & 10.6043 & $\mathrm{Y} / \mathrm{Y}$ \\
\hline 1014728 & $\begin{array}{lll}0100 & 20.75\end{array}$ & $\begin{array}{lll}-33 & 47 & 10.7\end{array}$ & 07 Oct 2001 & $17.67 \pm 0.03$ & $1.63 \pm 0.04$ & $0.05 \pm 0.04$ & 99.20 & $\ldots$ & 0.42 & 6 & 6.9959 & $\mathrm{Y} / \mathrm{Y}$ \\
\hline 1015134 & $\begin{array}{lll}01 & 00 & 22.97\end{array}$ & -334302.0 & 07 Oct 2001 & $17.78 \pm 0.03$ & $1.80 \pm 0.03$ & $-0.01 \pm 0.03$ & 91.50 & $\cdots$ & 0.56 & 7 & 3.5556 & $\mathrm{Y} / \mathrm{Y}$ \\
\hline 1015272 & $\begin{array}{lll}01 & 00 & 22.73\end{array}$ & -335122.6 & 07 Oct 2001 & $18.09 \pm 0.02$ & $1.40 \pm 0.03$ & $0.01 \pm 0.03$ & 101.90 & $\ldots$ & 0.66 & 7 & 12.7098 & $\mathrm{Y} / \mathrm{Y}$ \\
\hline 1015321 & $\begin{array}{lll}01 & 00 & 23.83\end{array}$ & $\begin{array}{lll}-33 & 42 & 17.3\end{array}$ & $\cdots$ & $17.56 \pm 0.02$ & $1.74 \pm 0.04$ & $0.03 \pm 0.03$ & 120.42 & 1.58 & $\cdots$ & $\cdots$ & 3.7771 & $\mathrm{~N}^{\mathrm{f}} / \mathrm{Y}$ \\
\hline 1015603 & $\begin{array}{lll}01 & 00 & 25.03\end{array}$ & -334228.0 & 07 Oct2001 & $18.36 \pm 0.03$ & $1.46 \pm 0.04$ & $0.00 \pm 0.04$ & 116.00 & $\cdots$ & 0.67 & 7 & 3.9898 & $\mathrm{Y} / \mathrm{Y}$ \\
\hline 1015645 & $\begin{array}{lll}01 & 00 & 25.09\end{array}$ & -334352.9 & $\ldots$ & $17.73 \pm 0.02$ & $1.78 \pm 0.04$ & $-0.01 \pm 0.03$ & 115.85 & 1.34 & $\cdots$ & $\ldots$ & 4.2733 & $\mathrm{~N}^{\mathrm{f}^{\prime}} / \mathrm{Y}$ \\
\hline 1015830 & $\begin{array}{lll}01 & 00 & 25.29\end{array}$ & -335050.5 & $\ldots$ & $17.39 \pm 0.02$ & $1.93 \pm 0.03$ & $-0.05 \pm 0.02$ & 107.88 & 3.71 & $\cdots$ & 8 & 12.1221 & $\mathrm{Y} / \mathrm{Y}$ \\
\hline 1016179 & 010027.85 & -334031.5 & $\ldots$ & $17.29 \pm 0.02$ & $1.79 \pm 0.03$ & $-0.26 \pm 0.03$ & -18.60 & 4.70 & $\ldots$ & $\ldots$ & 5.5783 & $\mathrm{~N} / \mathrm{N}$ \\
\hline 1016274 & 010028.10 & -334234.2 & 07 Oct 2001 & $17.84 \pm 0.02$ & $1.57 \pm 0.03$ & $0.02 \pm 0.03$ & 108.10 & $\cdots$ & 0.47 & 7 & 4.6147 & $\mathrm{Y} / \mathrm{Y}$ \\
\hline 1016796 & 010030.75 & -333730.4 & 07 Oct 2001 & $17.63 \pm 0.02$ & $1.64 \pm 0.03$ & $0.01 \pm 0.03$ & 88.10 & $\cdots$ & 0.83 & 7 & 9.1327 & $\mathrm{Y} / \mathrm{Y}$ \\
\hline 1017220 & $\begin{array}{lll}01 & 00 & 32.13\end{array}$ & -334418.5 & $\ldots$ & $17.57 \pm 0.02$ & $1.70 \pm 0.03$ & $0.00 \pm 0.03$ & 102.57 & 3.71 & $\cdots$ & 8 & 5.8329 & $\mathrm{Y} / \mathrm{Y}$ \\
\hline 1017304 & 010032.64 & -334230.5 & $\cdots$ & $17.68 \pm 0.02$ & $1.56 \pm 0.04$ & $-0.00 \pm 0.03$ & 112.02 & 2.83 & $\cdots$ & 8 & 5.5684 & $\mathrm{Y} / \mathrm{Y}$ \\
\hline 1017546 & 010034.03 & -333904.5 & $\ldots$ & $17.49 \pm 0.02$ & $2.02 \pm 0.03$ & $-0.01 \pm 0.03$ & 110.44 & 3.71 & $\cdots$ & 8 & 7.9170 & $\mathrm{Y} / \mathrm{Y}$ \\
\hline 1017864 & 010035.76 & $\begin{array}{lll}-33 & 37 & 54.2\end{array}$ & $\ldots$ & $17.64 \pm 0.02$ & $1.71 \pm 0.02$ & $0.01 \pm 0.02$ & 101.53 & 3.71 & $\ldots$ & 8 & 9.3705 & $\mathrm{Y} / \mathrm{Y}$ \\
\hline 1017977 & 010035.57 & $\begin{array}{lll}-33 & 45 & 06.2\end{array}$ & $\ldots$ & $17.64 \pm 0.02$ & $1.68 \pm 0.03$ & $0.02 \pm 0.03$ & 100.90 & 4.70 & $\ldots$ & $\ldots$ & 6.9359 & $\mathrm{Y} / \mathrm{Y}$ \\
\hline 1018123 & 010035.93 & $-3348 \quad 14.3$ & 13Nov2000 & $17.87 \pm 0.03$ & $1.64 \pm 0.03$ & $0.03 \pm 0.03$ & 98.60 & $\ldots$ & 0.73 & 7 & 9.8568 & $\mathrm{Y} / \mathrm{Y}$ \\
\hline 1018435 & 010038.06 & $\begin{array}{lll}-33 & 42 & 02.9\end{array}$ & $\ldots$ & $17.04 \pm 0.02$ & $1.87 \pm 0.03$ & $-0.30 \pm 0.02$ & 25.70 & 4.70 & $\ldots$ & $\ldots$ & 6.7790 & $\mathrm{~N} / \mathrm{N}$ \\
\hline 1018551 & $\begin{array}{lll}01 & 00 & 38.11\end{array}$ & -334816.7 & $\cdots$ & $17.49 \pm 0.02$ & $1.77 \pm 0.03$ & $0.02 \pm 0.03$ & 106.46 & 2.53 & $\cdots$ & $\cdots$ & 10.1773 & $\mathrm{Y} / \mathrm{Y}$ \\
\hline 1019002 & $\begin{array}{lll}01 & 00 & 39.44\end{array}$ & -335449.0 & 07 Oct 2001 & $18.40 \pm 0.03$ & $1.38 \pm 0.05$ & $0.03 \pm 0.04$ & 109.40 & $\cdots$ & 0.69 & 7 & 18.3108 & $\mathrm{Y} / \mathrm{Y}$ \\
\hline 1019402 & $\begin{array}{lll}01 & 00 & 42.23\end{array}$ & $\begin{array}{llll}-33 & 448.6\end{array}$ & $\cdots$ & $19.23 \pm 0.04$ & $1.29 \pm 0.05$ & $0.07 \pm 0.06$ & 90.45 & 1.51 & $\cdots$ & $\cdots$ & 7.9398 & $\mathrm{Y} / \mathrm{Y}$ \\
\hline 1019417 & $\begin{array}{llll}01 & 00 & 42.42\end{array}$ & $\begin{array}{lll}-33 & 43 & 09.0\end{array}$ & 07 Oct2001 & $17.28 \pm 0.02$ & $1.74 \pm 0.03$ & $0.02 \pm 0.02$ & 99.00 & $\cdots$ & 0.97 & 7 & 7.5935 & $\mathrm{Y} / \mathrm{Y}$ \\
\hline
\end{tabular}


Table 4-Continued

\begin{tabular}{|c|c|c|c|c|c|c|c|c|c|c|c|c|}
\hline ID & $\alpha(\mathrm{J} 2000.0)$ & $\delta(\mathrm{J} 2000.0)$ & UT Date ${ }^{\mathrm{a}}$ & $M$ & $M-T_{2}$ & $M-D$ & $v_{\text {hel }}{ }^{\mathrm{b}}$ & $\epsilon_{v_{\text {hel }}}^{c}$ & $C C P$ & $Q$ & $a\left({ }^{\prime}\right)$ & $\mathrm{P} / \mathrm{V}^{\mathrm{d}}$ \\
\hline 1019459 & 010042.49 & -334423.4 & 13 Nov 2000 & $17.52 \pm 0.02$ & $1.85 \pm 0.03$ & $-0.00 \pm 0.02$ & 97.80 & 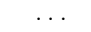 & 0.66 & 7 & 7.8853 & $\mathrm{Y} / \mathrm{Y}$ \\
\hline 1019945 & $0100 \quad 44.27$ & -334918.7 & $\cdots$ & $17.71 \pm 0.02$ & $1.71 \pm 0.03$ & $0.03 \pm 0.03$ & 114.07 & 3.71 & $\ldots$ & 8 & 12.0764 & $\mathrm{Y} / \mathrm{Y}$ \\
\hline 1020962 & 010049.36 & -334200.5 & $\ldots$ & $17.32 \pm 0.02$ & $1.84 \pm 0.03$ & $0.02 \pm 0.02$ & 114.31 & 2.83 & $\ldots$ & 8 & 9.1295 & $\mathrm{Y} / \mathrm{Y}$ \\
\hline 1021357 & 010050.86 & $\begin{array}{lll}-33 & 45 & 05.2\end{array}$ & $\cdots$ & $17.43 \pm 0.02$ & $1.97 \pm 0.03$ & $0.01 \pm 0.02$ & 109.51 & 3.71 & $\cdots$ & 8 & 9.8146 & $\mathrm{Y} / \mathrm{Y}$ \\
\hline 1021807 & 010052.74 & -334757.1 & $\ldots$ & $17.29 \pm 0.02$ & $2.23 \pm 0.02$ & $-0.22 \pm 0.02$ & -28.80 & 4.70 & $\ldots$ & $\ldots$ & 12.0223 & $\mathrm{~N} / \mathrm{N}$ \\
\hline 1021965 & 010054.17 & $-3340 \quad 14.5$ & 07 Oct 2001 & $17.46 \pm 0.02$ & $1.81 \pm 0.03$ & $0.02 \pm 0.02$ & 108.20 & $\ldots$ & 1.14 & 7 & 10.7624 & $\mathrm{Y} / \mathrm{Y}$ \\
\hline 1022096 & 010053.69 & -335227.4 & 07 Oct 2001 & $17.82 \pm 0.02$ & $1.57 \pm 0.03$ & $0.03 \pm 0.03$ & 108.20 & $\ldots$ & 1.14 & 7 & 16.7099 & $\mathrm{Y} / \mathrm{Y}$ \\
\hline 1023206 & 010059.03 & -335111.6 & 07 Oct 2001 & $18.34 \pm 0.02$ & $1.40 \pm 0.03$ & $0.02 \pm 0.03$ & 105.00 & $\ldots$ & 0.60 & 7 & 16.0091 & $\mathrm{Y} / \mathrm{Y}$ \\
\hline 2002885 & 010124.59 & -333834.3 & 07 Oct 2001 & $17.44 \pm 0.03$ & $1.91 \pm 0.04$ & $0.02 \pm 0.04$ & 111.70 & $\ldots$ & 0.45 & 6 & 17.6396 & $\mathrm{Y} / \mathrm{Y}$ \\
\hline 2003994 & 010130.16 & -334616.5 & 07 Oct 2001 & $18.19 \pm 0.02$ & $1.44 \pm 0.05$ & $0.09 \pm 0.04$ & 111.90 & $\ldots$ & 0.53 & 7 & 18.0374 & $\mathrm{Y} / \mathrm{Y}$ \\
\hline 2005462 & 010139.56 & $-3342 \quad 15.6$ & 07 Oct 2001 & $18.37 \pm 0.04$ & $1.47 \pm 0.07$ & $0.09 \pm 0.05$ & 113.70 & $\ldots$ & 0.73 & 7 & 19.5377 & $\mathrm{Y} / \mathrm{Y}$ \\
\hline 2007151 & 010149.32 & -334302.5 & 07 Oct 2001 & $18.65 \pm 0.04$ & $1.27 \pm 0.06$ & $0.06 \pm 0.06$ & 115.00 & $\ldots$ & 0.42 & 5 & 21.5164 & $\mathrm{Y} / \mathrm{Y}$ \\
\hline 2007352 & 010149.42 & -335409.8 & $\ldots$ & $17.22 \pm 0.02$ & $1.76 \pm 0.05$ & $0.06 \pm 0.03$ & 117.64 & 1.40 & $\ldots$ & 8 & 26.3928 & $\mathrm{Y} / \mathrm{Y}$ \\
\hline 2008582 & $\begin{array}{lll}01 & 01 & 57.75\end{array}$ & -334250.6 & 07Oct2001 & $18.90 \pm 0.05$ & $1.44 \pm 0.07$ & $0.08 \pm 0.07$ & 105.10 & $\ldots$ & 1.09 & 7 & 23.2820 & $\mathrm{Y} / \mathrm{Y}$ \\
\hline 2012883 & $\begin{array}{lll}01 & 02 & 23.60\end{array}$ & -334343.8 & $28 \mathrm{Jan} 2004$ & $17.33 \pm 0.03$ & $1.78 \pm 0.05$ & $0.04 \pm 0.04$ & 112.90 & $\ldots$ & 0.71 & 6 & 28.6434 & $\mathrm{Y} / \mathrm{Y}$ \\
\hline 3001748 & 005918.60 & -335941.1 & 07 Oct 2001 & $18.90 \pm 0.03$ & $1.24 \pm 0.04$ & $0.03 \pm 0.04$ & 113.00 & $\ldots$ & 0.32 & 6 & 26.5294 & $\mathrm{Y} / \mathrm{Y}$ \\
\hline 3003666 & 005930.00 & -340010.8 & 07 Oct 2001 & $19.18 \pm 0.07$ & $1.27 \pm 0.09$ & $0.05 \pm 0.10$ & 117.30 & $\ldots$ & 0.44 & 5 & 26.3113 & $\mathrm{Y} / \mathrm{Y}$ \\
\hline 3005776 & 005943.16 & -335646.5 & $\ldots$ & $17.24 \pm 0.03$ & $1.74 \pm 0.04$ & $0.02 \pm 0.04$ & 105.35 & 3.71 & $\ldots$ & 8 & 20.8182 & $\mathrm{Y} / \mathrm{Y}$ \\
\hline 3007270 & 005951.18 & -340332.0 & 07 Oct2001 & $19.96 \pm 0.11$ & $0.49 \pm 0.13$ & $0.04 \pm 0.17$ & 108.30 & $\cdots$ & 1.03 & 7 & 29.9402 & $\mathrm{Y}^{\mathrm{g}} / \mathrm{Y}$ \\
\hline 3008933 & 010001.14 & -335921.1 & 07Oct 2001 & $18.76 \pm 0.04$ & $1.26 \pm 0.07$ & $0.10 \pm 0.06$ & 119.40 & $\ldots$ & 0.32 & 5 & 23.7732 & $\mathrm{Y} / \mathrm{Y}$ \\
\hline 3010660 & 010010.92 & -340134.6 & $\ldots$ & $17.70 \pm 0.04$ & $1.64 \pm 0.06$ & $0.05 \pm 0.05$ & 102.56 & 2.83 & $\ldots$ & 8 & 26.8320 & $\mathrm{Y} / \mathrm{Y}$ \\
\hline 3012313 & 010021.09 & -335627.8 & $\ldots$ & $17.86 \pm 0.03$ & $1.49 \pm 0.05$ & $0.04 \pm 0.04$ & 90.79 & 2.72 & $\cdots$ & 8 & 19.7077 & $\mathrm{Y} / \mathrm{Y}$ \\
\hline 3012794 & 010023.78 & -335818.5 & 07 Oct 2001 & $18.27 \pm 0.02$ & $1.41 \pm 0.05$ & $-0.01 \pm 0.04$ & 98.00 & $\ldots$ & 0.76 & 7 & 22.3559 & $\mathrm{Y} / \mathrm{Y}$ \\
\hline 3013450 & $\begin{array}{lll}01 & 00 & 26.22\end{array}$ & $-3411 \quad 20.3$ & 07Oct2001 & $17.51 \pm 0.03$ & $1.69 \pm 0.04$ & $0.01 \pm 0.04$ & 118.60 & $\ldots$ & 0.89 & 7 & 40.7306 & $\mathrm{Y} / \mathrm{Y}$ \\
\hline 3015968 & 010041.51 & -335530.1 & 07 Oct 2001 & $18.66 \pm 0.04$ & $1.34 \pm 0.06$ & $-0.00 \pm 0.06$ & 117.00 & $\ldots$ & 0.55 & 7 & 19.3539 & $\mathrm{Y} / \mathrm{Y}$ \\
\hline 3016642 & 010044.58 & $-3400 \quad 33.9$ & 07 Oct 2001 & $18.03 \pm 0.03$ & $1.47 \pm 0.05$ & $0.00 \pm 0.04$ & 103.00 & $\ldots$ & 0.24 & 5 & 26.2969 & $\mathrm{Y} / \mathrm{Y}$ \\
\hline 4020490 & 010103.08 & -333613.6 & $\ldots$ & $17.01 \pm 0.02$ & $2.23 \pm 0.03$ & $-0.22 \pm 0.03$ & 15.40 & 4.70 & $\ldots$ & $\ldots$ & 15.3335 & $\mathrm{~N} / \mathrm{N}$ \\
\hline 4020503 & $\begin{array}{llll}01 & 01 & 02.87\end{array}$ & $\begin{array}{lll}-33 & 38 & 52.1\end{array}$ & 07Oct2001 & $17.33 \pm 0.03$ & $1.91 \pm 0.04$ & $-0.01 \pm 0.03$ & 97.00 & $\ldots$ & 0.66 & 7 & 13.2474 & $\mathrm{Y} / \mathrm{Y}$ \\
\hline 4020870 & $\begin{array}{lll}01 & 01 & 04.48\end{array}$ & $-3341 \quad 16.7$ & 07 Oct 2001 & $18.24 \pm 0.04$ & $1.55 \pm 0.06$ & $0.07 \pm 0.05$ & 111.90 & $\ldots$ & 0.59 & 7 & 12.4383 & $\mathrm{Y} / \mathrm{Y}$ \\
\hline 4021825 & 010108.55 & -334534.9 & 07 Oct 2001 & $17.42 \pm 0.01$ & $1.85 \pm 0.03$ & $0.03 \pm 0.02$ & 113.30 & $\ldots$ & 0.84 & 7 & 13.4928 & $\mathrm{Y} / \mathrm{Y}$ \\
\hline 4021850 & $\begin{array}{lll}01 & 01 & 09.78\end{array}$ & -333420.1 & $\ldots$ & $17.24 \pm 0.01$ & $2.85 \pm 0.02$ & $-0.10 \pm 0.02$ & 22.00 & 4.70 & $\ldots$ & $\ldots$ & 18.1988 & $\mathrm{~N} / \mathrm{N}$ \\
\hline 4021889 & $\begin{array}{lll}01 & 01 & 09.09\end{array}$ & -334324.1 & 07 Oct 2001 & $17.60 \pm 0.02$ & $1.80 \pm 0.03$ & $0.01 \pm 0.02$ & 83.70 & $\ldots$ & 0.70 & 7 & 13.1427 & $\mathrm{Y} / \mathrm{Y}$ \\
\hline 4023044 & $0101 \quad 15.08$ & -334241.8 & 07 Oct 2001 & $17.89 \pm 0.02$ & $1.56 \pm 0.03$ & $0.05 \pm 0.03$ & 106.50 & $\ldots$ & 0.69 & 7 & 14.3976 & $\mathrm{Y} / \mathrm{Y}$ \\
\hline 4023922 & $0101 \quad 19.26$ & -334541.7 & 07 Oct 2001 & $17.77 \pm 0.02$ & $1.62 \pm 0.04$ & $0.04 \pm 0.03$ & 83.00 & $\ldots$ & 0.50 & 6 & 15.6665 & $\mathrm{Y} / \mathrm{Y}$ \\
\hline 5001661 & $0100 \quad 57.80$ & -340442.8 & $\ldots$ & $17.36 \pm 0.02$ & $1.78 \pm 0.03$ & $0.01 \pm 0.02$ & 113.32 & 1.40 & $\ldots$ & 8 & 32.6238 & $\mathrm{Y} / \mathrm{Y}$ \\
\hline 5003374 & $\begin{array}{lll}01 & 01 & 09.07\end{array}$ & -335915.9 & 07Oct2001 & $19.18 \pm 0.05$ & $1.30 \pm 0.07$ & $0.03 \pm 0.08$ & 102.40 & $\ldots$ & 0.53 & 7 & 26.4316 & $\mathrm{Y} / \mathrm{Y}$ \\
\hline 5004927 & $\begin{array}{llll}01 & 01 & 17.91\end{array}$ & -340500.8 & 07Oct2001 & $18.84 \pm 0.06$ & $1.34 \pm 0.08$ & $0.05 \pm 0.08$ & 111.10 & $\ldots$ & 0.37 & 7 & 34.4293 & $\mathrm{Y} / \mathrm{Y}$ \\
\hline 5006172 & 010125.19 & -341020.6 & 07 Oct 2001 & $17.44 \pm 0.03$ & $1.04 \pm 0.06$ & $0.06 \pm 0.04$ & 219.30 & $\ldots$ & 0.60 & 7 & 41.8727 & $\mathrm{~N} / \mathrm{N}$ \\
\hline 5007253 & 010132.96 & -335904.1 & 07 Oct2001 & $18.44 \pm 0.03$ & $1.29 \pm 0.06$ & $0.04 \pm 0.05$ & 105.70 & $\ldots$ & 0.69 & 7 & 28.8059 & $\mathrm{Y} / \mathrm{Y}$ \\
\hline 5009742 & $\begin{array}{lll}01 & 01 & 47.24\end{array}$ & -340809.2 & 07 Oct2001 & $20.26 \pm 0.13$ & $0.51 \pm 0.16$ & $0.18 \pm 0.19$ & 125.30 & $\ldots$ & 0.90 & 7 & 40.9780 & $\mathrm{~N}^{\mathrm{h}} / \mathrm{Y}$ \\
\hline 5011134 & 010156.15 & -340511.0 & 07 Oct 2001 & $17.24 \pm 0.03$ & $1.26 \pm 0.06$ & $-0.05 \pm 0.05$ & -67.20 & $\ldots$ & 0.40 & 5 & 38.4225 & $\mathrm{~N} / \mathrm{N}$ \\
\hline 5012996 & $\begin{array}{lll}01 & 02 & 08.03\end{array}$ & -335750.6 & 07 Oct 2001 & $17.79 \pm 0.03$ & $1.54 \pm 0.06$ & $0.04 \pm 0.04$ & 106.50 & $\ldots$ & 0.45 & 7 & 32.5062 & $\mathrm{Y} / \mathrm{Y}$ \\
\hline 5014056 & $\begin{array}{lll}01 & 02 & 13.25\end{array}$ & -340601.5 & 07 Oct2001 & $16.77 \pm 0.03$ & $1.25 \pm 0.05$ & $0.05 \pm 0.03$ & -109.90 & $\cdots$ & 0.69 & 7 & 41.4127 & $\mathrm{~N} / \mathrm{N}$ \\
\hline 5016783 & 010228.66 & $\begin{array}{lll}-34 & 06 & 09.2\end{array}$ & 07 Oct2001 & $18.50 \pm 0.02$ & $1.35 \pm 0.03$ & $0.06 \pm 0.03$ & 84.20 & $\cdots$ & 0.39 & 7 & 43.5382 & $\mathrm{Y} / \mathrm{Y}$ \\
\hline 5017300 & 010231.01 & $-34 \quad 1048.3$ & 07 Oct2001 & $19.64 \pm 0.04$ & $1.06 \pm 0.07$ & $0.04 \pm 0.06$ & 96.40 & $\ldots$ & 0.79 & 7 & 48.8556 & $\mathrm{~N}^{\mathrm{i}} / \mathrm{Y}$ \\
\hline 6007372 & $\begin{array}{lll}01 & 03 & 13.28\end{array}$ & -335001.1 & 28Jan2004 & $17.24 \pm 0.03$ & $1.81 \pm 0.05$ & $0.05 \pm 0.04$ & 86.04 & $\cdots$ & 0.84 & 6 & 39.8802 & $\mathrm{Y} / \mathrm{Y}$ \\
\hline 6009453 & 010325.78 & -335359.3 & 27Jan2004 & $17.46 \pm 0.02$ & $1.81 \pm 0.03$ & $0.03 \pm 0.02$ & 126.59 & $\ldots$ & 0.98 & 7 & 43.8453 & $\mathrm{Y} / \mathrm{Y}$ \\
\hline 6015626 & 010404.01 & $-3340 \quad 02.2$ & 28Jan2004 & $18.61 \pm 0.04$ & $1.36 \pm 0.06$ & $0.09 \pm 0.05$ & 115.11 & $\ldots$ & 1.11 & 7 & 49.9468 & $\mathrm{Y} / \mathrm{Y}$ \\
\hline 7002726 & 010245.46 & $\begin{array}{lll}-34 & 02 & 13.8\end{array}$ & 31Dec2004 & $18.46 \pm 0.03$ & $1.29 \pm 0.05$ & $0.08 \pm 0.05$ & 112.12 & $\ldots$ & 0.64 & 6 & 42.2110 & $\mathrm{Y} / \mathrm{Y}$ \\
\hline 7007699 & $0103 \quad 19.52$ & -335458.9 & 27Jan2004 & $18.23 \pm 0.03$ & $1.43 \pm 0.04$ & $0.02 \pm 0.04$ & 109.12 & $\ldots$ & 0.74 & 7 & 43.1188 & $\mathrm{Y} / \mathrm{Y}$ \\
\hline 7010887 & 010339.21 & -340514.7 & 10 Oct 2001 & $17.90 \pm 0.03$ & $1.12 \pm 0.05$ & $0.07 \pm 0.04$ & -410.40 & $\ldots$ & 0.25 & 4 & 53.4052 & $\mathrm{~N} / \mathrm{N}$ \\
\hline 7013244 & 010353.62 & -341348.0 & 27Jan2004 & $17.35 \pm 0.02$ & $1.78 \pm 0.02$ & $0.01 \pm 0.02$ & 118.90 & $\ldots$ & 0.89 & 7 & 63.0975 & $\mathrm{Y} / \mathrm{Y}$ \\
\hline 7015937 & $\begin{array}{lll}01 & 04 & 10.82\end{array}$ & -335507.0 & $\ldots$ & $19.11 \pm 0.03$ & $1.28 \pm 0.04$ & $0.02 \pm 0.04$ & 71.58 & 2.12 & $\ldots$ & 8 & 53.0991 & $\mathrm{Y} / \mathrm{N}^{\mathrm{j}}$ \\
\hline 9018559 & $\begin{array}{lll}01 & 02 & 33.21\end{array}$ & $\begin{array}{lll}-33 & 14 & 13.1\end{array}$ & 28Jan2004 & $18.45 \pm 0.03$ & $1.28 \pm 0.03$ & $0.05 \pm 0.03$ & 114.02 & $\ldots$ & 0.79 & 6 & 51.9248 & $\mathrm{Y} / \mathrm{Y}$ \\
\hline 9020273 & $\begin{array}{lll}01 & 02 & 41.18\end{array}$ & $-3318 \quad 30.5$ & 31Dec2004 & $18.63 \pm 0.03$ & $1.27 \pm 0.03$ & $0.08 \pm 0.04$ & 100.99 & $\ldots$ & 0.64 & 7 & 48.3107 & $\mathrm{Y} / \mathrm{Y}$ \\
\hline 10002876 & $\begin{array}{lll}01 & 02 & 39.17\end{array}$ & $-34 \quad 1809.3$ & 27Jan2004 & $18.34 \pm 0.03$ & $1.39 \pm 0.04$ & $0.07 \pm 0.04$ & 110.58 & $\ldots$ & 0.72 & 5 & 58.2527 & $\mathrm{Y} / \mathrm{Y}$ \\
\hline 14002354 & 010438.62 & $-3408 \quad 20.2$ & 31Dec2004 & $18.99 \pm 0.04$ & $1.31 \pm 0.07$ & $0.11 \pm 0.05$ & 117.03 & $\ldots$ & 0.77 & 6 & 65.8121 & $\mathrm{Y} / \mathrm{Y}$ \\
\hline 14004104 & 010501.38 & $-3408 \quad 20.2$ & 31Dec2004 & $18.39 \pm 0.04$ & $1.21 \pm 0.07$ & $0.04 \pm 0.05$ & -56.04 & $\ldots$ & 0.84 & 7 & 69.7935 & $\mathrm{Y} / \mathrm{N}$ \\
\hline 14007314 & $\begin{array}{lll}01 & 05 & 40.98\end{array}$ & $-3401 \quad 28.2$ & $10 O \operatorname{ct} 2001$ & $20.59 \pm 0.07$ & $1.49 \pm 0.11$ & $0.14 \pm 0.11$ & 208.70 & $\ldots$ & 0.41 & 5 & 73.3307 & $\mathrm{~N} / \mathrm{N}$ \\
\hline 15003281 & $0104 \quad 44.81$ & -342342.4 & 31Dec2004 & $19.38 \pm 0.04$ & $1.19 \pm 0.06$ & $0.05 \pm 0.06$ & -9.50 & $\ldots$ & 0.53 & 4 & 80.0073 & $\mathrm{Y} / \mathrm{N}$ \\
\hline 15005015 & $\begin{array}{lll}01 & 05 & 04.08\end{array}$ & -342616.9 & 10 Oct 2001 & $20.26 \pm 0.06$ & $0.42 \pm 0.12$ & $0.24 \pm 0.08$ & 98.10 & $\ldots$ & 0.32 & 4 & 85.2565 & $\mathrm{Y}^{\mathrm{g}} / \mathrm{Y}$ \\
\hline 15005725 & $0105 \quad 11.81$ & -342302.8 & 27Jan2004 & $18.80 \pm 0.04$ & $1.25 \pm 0.06$ & $0.13 \pm 0.04$ & 95.78 & $\ldots$ & 0.62 & 5 & 83.3263 & $\mathrm{Y} / \mathrm{Y}$ \\
\hline 18000837 & 010209.75 & -345135.4 & 30Dec2004 & $19.59 \pm 0.04$ & $1.29 \pm 0.06$ & $0.01 \pm 0.06$ & 48.20 & $\ldots$ & 0.51 & 4 & 99.9129 & $\mathrm{Y} / \mathrm{N}$ \\
\hline 20000170 & 010243.58 & -332734.2 & 31Dec2004 & $18.68 \pm 0.04$ & $1.30 \pm 0.05$ & $0.04 \pm 0.05$ & 117.45 & $\ldots$ & 0.73 & 5 & 40.1150 & $\mathrm{Y} / \mathrm{Y}$ \\
\hline 21000544 & 010331.93 & $\begin{array}{lll}-33 & 01 & 18.3\end{array}$ & 31Dec2004 & $19.23 \pm 0.05$ & $1.11 \pm 0.07$ & $0.07 \pm 0.07$ & 150.71 & $\ldots$ & 0.51 & 5 & 74.5104 & $\mathrm{Y} / \mathrm{N}$ \\
\hline 24000732 & $\begin{array}{lll}01 & 05 & 03.28\end{array}$ & -331544.2 & 27Jan2004 & $17.84 \pm 0.02$ & $1.35 \pm 0.03$ & $0.04 \pm 0.03$ & 53.96 & $\cdots$ & 0.82 & 5 & 74.5730 & $\mathrm{Y} / \mathrm{N}$ \\
\hline 28000143 & 005925.28 & $\begin{array}{lll}-33 & 26 & 31.2\end{array}$ & 07Oct2001 & $19.76 \pm 0.05$ & $1.18 \pm 0.06$ & $0.07 \pm 0.07$ & 104.00 & $\cdots$ & 0.43 & 7 & 24.0353 & $\mathrm{Y} / \mathrm{Y}$ \\
\hline
\end{tabular}


Table 4-Continued

\begin{tabular}{|c|c|c|c|c|c|c|c|c|c|c|c|c|}
\hline ID & $\alpha(\mathrm{J} 2000.0)$ & $\delta(\mathrm{J} 2000.0)$ & $\mathrm{UT} \mathrm{Date}^{\mathrm{a}}$ & $M$ & $M-T_{2}$ & $M-D$ & $v_{\text {hel }}{ }^{\mathrm{b}}$ & $\epsilon_{v_{\text {hel }}}{ }^{\mathrm{c}}$ & $C C P$ & $Q$ & $a\left(^{\prime}\right)$ & $\mathrm{P} / \mathrm{V}^{\mathrm{d}}$ \\
\hline 28000278 & 005936.98 & -333028.2 & $\cdots$ & $17.89 \pm 0.02$ & $1.59 \pm 0.03$ & $0.03 \pm 0.03$ & 107.18 & 3.71 & $\cdots$ & 8 & 18.0372 & $\mathrm{Y} / \mathrm{Y}$ \\
\hline 28000376 & 005944.12 & -332811.6 & $\ldots$ & $17.81 \pm 0.02$ & $1.60 \pm 0.03$ & $0.03 \pm 0.03$ & 108.37 & 3.71 & $\cdots$ & 8 & 20.7982 & $\mathrm{Y} / \mathrm{Y}$ \\
\hline 29000568 & 010130.33 & -332255.3 & 28Jan2004 & $17.34 \pm 0.02$ & $1.69 \pm 0.03$ & $0.07 \pm 0.03$ & 113.42 & $\cdots$ & 0.35 & 5 & 33.7818 & $\mathrm{Y} / \mathrm{Y}$ \\
\hline 29000872 & $\begin{array}{lll}01 & 02 & 03.69\end{array}$ & $\begin{array}{lll}-33 & 25 & 07.5\end{array}$ & 28Jan2004 & $17.39 \pm 0.02$ & $1.72 \pm 0.03$ & $0.09 \pm 0.03$ & 111.92 & $\cdots$ & 0.41 & 5 & 35.7978 & $\mathrm{Y} / \mathrm{Y}$ \\
\hline 29001068 & $\begin{array}{lll}01 & 02 & 22.24\end{array}$ & $\begin{array}{lll}-33 & 23 & 04.3\end{array}$ & 27Jan2004 & $17.29 \pm 0.02$ & $1.77 \pm 0.03$ & $0.04 \pm 0.03$ & 120.75 & $\ldots$ & 1.21 & 7 & 40.7253 & $\mathrm{Y} / \mathrm{Y}$ \\
\hline 30000043 & 005732.71 & $\begin{array}{lll}-33 & 34 & 07.1\end{array}$ & 28Jan2004 & $17.53 \pm 0.02$ & $1.61 \pm 0.03$ & $0.05 \pm 0.04$ & 113.22 & $\cdots$ & 0.71 & 7 & 33.6736 & $\mathrm{Y} / \mathrm{Y}$ \\
\hline 30000294 & $0058 \quad 11.50$ & -333047.0 & 07 Oct 2001 & $19.14 \pm 0.05$ & $1.24 \pm 0.07$ & $0.07 \pm 0.09$ & 99.10 & $\cdots$ & 0.77 & 7 & 28.5906 & $\mathrm{Y} / \mathrm{Y}$ \\
\hline 30000318 & $\begin{array}{lll}00 & 58 & 15.72\end{array}$ & $\begin{array}{lll}-33 & 27 & 16.1\end{array}$ & 28Jan 2004 & $17.35 \pm 0.03$ & $1.72 \pm 0.04$ & $0.05 \pm 0.05$ & 127.28 & $\cdots$ & 0.96 & 7 & 30.9852 & $\mathrm{Y} / \mathrm{Y}$ \\
\hline 30000352 & $0058 \quad 19.23$ & -333441.0 & 07 Oct 2001 & $19.01 \pm 0.04$ & $1.21 \pm 0.04$ & $0.07 \pm 0.05$ & 103.10 & $\cdots$ & 0.27 & 5 & 24.5066 & $\mathrm{Y} / \mathrm{Y}$ \\
\hline 30000378 & 005823.29 & $\begin{array}{lll}-33 & 29 & 30.7\end{array}$ & 13 Nov 2000 & $18.43 \pm 0.03$ & $0.99 \pm 0.05$ & $0.08 \pm 0.06$ & 104.40 & $\ldots$ & 1.23 & 7 & 27.7516 & $\mathrm{~N} / \mathrm{Y}$ \\
\hline 30000548 & $0058 \quad 44.70$ & -333048.8 & 07 Oct 2001 & $18.40 \pm 0.04$ & $1.40 \pm 0.05$ & $0.10 \pm 0.06$ & 102.10 & $\ldots$ & 0.54 & 7 & 23.3100 & $\mathrm{Y} / \mathrm{Y}$ \\
\hline 31000074 & 005735.53 & -334121.7 & 07Oct2001 & $18.05 \pm 0.03$ & $1.53 \pm 0.04$ & $0.08 \pm 0.06$ & 86.20 & $\cdots$ & 0.43 & 5 & 31.3343 & $\mathrm{Y} / \mathrm{Y}$ \\
\hline 31000176 & 005750.05 & -334004.6 & $\cdots$ & $17.22 \pm 0.03$ & $1.72 \pm 0.04$ & $0.01 \pm 0.05$ & 110.24 & 1.02 & $\cdots$ & 8 & 28.4093 & $\mathrm{Y} / \mathrm{Y}$ \\
\hline 31000178 & 005749.60 & -334447.7 & 07 Oct 2001 & $18.93 \pm 0.04$ & $1.33 \pm 0.05$ & $0.08 \pm 0.08$ & 112.60 & $\cdots$ & 0.40 & 6 & 28.7204 & $\mathrm{Y} / \mathrm{Y}$ \\
\hline 31000255 & $0058 \quad 02.92$ & -334906.5 & $\ldots$ & $17.24 \pm 0.03$ & $1.76 \pm 0.04$ & $0.07 \pm 0.05$ & 110.18 & 3.71 & $\cdots$ & 8 & 27.6132 & $\mathrm{Y} / \mathrm{Y}$ \\
\hline 31000262 & 005804.06 & -335041.4 & $\cdots$ & $17.95 \pm 0.03$ & $1.51 \pm 0.04$ & $0.06 \pm 0.06$ & 80.91 & 2.07 & $\cdots$ & 8 & 28.3082 & $\mathrm{Y} / \mathrm{Y}$ \\
\hline 31000281 & $0058 \quad 05.50$ & $-3355 \quad 16.2$ & 13 Nov 2000 & $17.89 \pm 0.02$ & $1.46 \pm 0.03$ & $0.08 \pm 0.03$ & 113.60 & $\ldots$ & 1.23 & 7 & 31.4734 & $\mathrm{Y} / \mathrm{Y}$ \\
\hline 31000357 & $\begin{array}{llll}00 & 58 & 19.48\end{array}$ & -334300.4 & 07 Oct 2001 & $18.58 \pm 0.04$ & $1.33 \pm 0.06$ & $0.04 \pm 0.07$ & 98.40 & $\ldots$ & 0.59 & 7 & 22.2276 & $\mathrm{Y} / \mathrm{Y}$ \\
\hline 31000451 & 005833.30 & -334747.6 & 07 Oct 2001 & $19.01 \pm 0.05$ & $1.32 \pm 0.07$ & $0.06 \pm 0.09$ & 101.40 & $\cdots$ & 0.52 & 6 & 20.9535 & $\mathrm{Y} / \mathrm{Y}$ \\
\hline 31000453 & 005833.75 & $\begin{array}{lll}-33 & 43 & 18.7\end{array}$ & 13 Nov 2000 & $17.66 \pm 0.03$ & $1.64 \pm 0.04$ & $0.05 \pm 0.05$ & 101.50 & $\cdots$ & 1.12 & 7 & 19.2886 & $\mathrm{Y} / \mathrm{Y}$ \\
\hline 31000458 & 005833.58 & -334939.6 & $\cdots$ & $17.96 \pm 0.03$ & $1.55 \pm 0.05$ & $0.08 \pm 0.06$ & 106.80 & 3.71 & $\cdots$ & 8 & 22.0682 & $\mathrm{Y} / \mathrm{Y}$ \\
\hline 31000504 & 005839.06 & $-3345 \quad 52.4$ & 07 Oct 2001 & $18.49 \pm 0.04$ & $1.38 \pm 0.05$ & $0.02 \pm 0.07$ & 118.20 & $\cdots$ & 0.55 & 7 & 18.8837 & $\mathrm{Y} / \mathrm{Y}$ \\
\hline 31000512 & 005839.93 & $\begin{array}{lll}-33 & 48 & 09.7\end{array}$ & 07 Oct2001 & $18.75 \pm 0.04$ & $1.25 \pm 0.06$ & $0.02 \pm 0.07$ & 98.80 & $\cdots$ & 0.37 & 5 & 19.8858 & $\mathrm{Y} / \mathrm{Y}$ \\
\hline 31000522 & 005841.29 & -335149.4 & $\ldots$ & $17.35 \pm 0.02$ & $1.76 \pm 0.03$ & $0.04 \pm 0.03$ & 115.99 & 3.71 & $\cdots$ & 8 & 22.3976 & $\mathrm{Y} / \mathrm{Y}$ \\
\hline 31000535 & $0058 \quad 42.57$ & -334833.3 & $\cdots$ & $17.29 \pm 0.03$ & $1.80 \pm 0.04$ & $0.01 \pm 0.05$ & 121.09 & 1.39 & $\ldots$ & 8 & 19.6359 & $\mathrm{Y} / \mathrm{Y}$ \\
\hline 31000550 & 005843.51 & $\begin{array}{lll}-33 & 50 & 30.7\end{array}$ & $\cdots$ & $18.47 \pm 0.04$ & $1.48 \pm 0.05$ & $0.09 \pm 0.07$ & 99.68 & 3.71 & $\ldots$ & 8 & 20.9087 & $\mathrm{Y} / \mathrm{Y}$ \\
\hline 31000554 & 005844.68 & -334156.1 & 07 Oct2001 & $17.28 \pm 0.03$ & $1.76 \pm 0.04$ & $0.05 \pm 0.05$ & 117.40 & $\cdots$ & 1.14 & 7 & 16.9310 & $\mathrm{Y} / \mathrm{Y}$ \\
\hline 31000580 & 005846.43 & -334547.9 & 07 Oct 2001 & $18.51 \pm 0.04$ & $1.39 \pm 0.05$ & $0.01 \pm 0.07$ & 105.40 & $\cdots$ & 0.57 & 7 & 17.3669 & $\mathrm{Y} / \mathrm{Y}$ \\
\hline 31000589 & 005846.71 & -335336.8 & $\ldots$ & $17.72 \pm 0.02$ & $1.62 \pm 0.03$ & $0.05 \pm 0.03$ & 113.78 & 3.71 & $\ldots$ & 8 & 23.1972 & $\mathrm{Y} / \mathrm{Y}$ \\
\hline 31000691 & 005856.61 & -333620.8 & 13 Nov 2000 & $18.32 \pm 0.04$ & $1.33 \pm 0.05$ & $-0.02 \pm 0.06$ & 114.20 & $\cdots$ & 1.19 & 7 & 16.6520 & $\mathrm{Y} / \mathrm{Y}$ \\
\hline 31000695 & 005856.85 & $\begin{array}{lll}-33 & 37 & 20.7\end{array}$ & 13 Nov 2000 & $18.44 \pm 0.03$ & $1.30 \pm 0.05$ & $0.03 \pm 0.06$ & 109.20 & $\cdots$ & 1.07 & 7 & 15.9501 & $\mathrm{Y} / \mathrm{Y}$ \\
\hline 31000730 & 005858.86 & -333902.6 & 13 Nov 2000 & $18.36 \pm 0.04$ & $1.30 \pm 0.05$ & $0.04 \pm 0.07$ & 12.00 & $\cdots$ & 0.81 & 7 & 14.6763 & $\mathrm{Y} / \mathrm{N}$ \\
\hline 31000788 & 005901.33 & -335431.5 & 07 Oct 2001 & $18.16 \pm 0.02$ & $1.44 \pm 0.03$ & $-0.00 \pm 0.03$ & 101.50 & $\cdots$ & 0.59 & 7 & 22.0496 & $\mathrm{Y} / \mathrm{Y}$ \\
\hline 31000795 & 005902.34 & $\begin{array}{lll}-33 & 47 & 06.2\end{array}$ & $\cdots$ & $17.28 \pm 0.03$ & $1.37 \pm 0.04$ & $-0.24 \pm 0.05$ & 51.00 & 4.70 & $\cdots$ & $\cdots$ & 14.9526 & $\mathrm{~N} / \mathrm{N}$ \\
\hline 31000807 & $\begin{array}{lll}00 & 59 & 02.82\end{array}$ & -334738.6 & $\cdots$ & $16.84 \pm 0.03$ & $1.50 \pm 0.04$ & $-0.31 \pm 0.05$ & 37.70 & 4.70 & $\cdots$ & $\cdots$ & 15.2325 & $\mathrm{~N} / \mathrm{N}$ \\
\hline 31000811 & $\begin{array}{lll}00 & 59 & 02.80\end{array}$ & -335049.5 & $\cdots$ & $17.54 \pm 0.03$ & $1.38 \pm 0.05$ & $-0.02 \pm 0.05$ & 3.58 & 2.07 & $\cdots$ & 8 & 17.9429 & $\mathrm{~N} / \mathrm{N}$ \\
\hline 31000813 & 005903.41 & $-3345 \quad 50.1$ & 07 Oct 2001 & $18.62 \pm 0.04$ & $1.30 \pm 0.05$ & $0.07 \pm 0.07$ & 102.50 & $\cdots$ & 0.65 & 7 & 13.9964 & $\mathrm{Y} / \mathrm{Y}$ \\
\hline 31000815 & 005904.03 & $\begin{array}{lll}-33 & 40 & 31.4\end{array}$ & 07 Oct 2001 & $18.25 \pm 0.04$ & $1.39 \pm 0.05$ & $0.02 \pm 0.06$ & 97.50 & $\cdots$ & 0.66 & 7 & 13.1258 & $\mathrm{Y} / \mathrm{Y}$ \\
\hline 31000822 & 005904.30 & -334405.9 & $\cdots$ & $17.74 \pm 0.03$ & $1.66 \pm 0.04$ & $0.05 \pm 0.05$ & 108.31 & 3.71 & $\cdots$ & 8 & 13.1136 & $\mathrm{Y} / \mathrm{Y}$ \\
\hline 31000824 & 005904.79 & -333844.2 & $\cdots$ & $17.07 \pm 0.03$ & $1.88 \pm 0.04$ & $0.01 \pm 0.05$ & 109.93 & 1.60 & $\cdots$ & $\cdots$ & 13.6755 & $\mathrm{~N}^{\mathrm{e}} / \mathrm{Y}$ \\
\hline 32000206 & 005755.07 & -335940.9 & 28Jan2004 & $17.39 \pm 0.03$ & $1.73 \pm 0.04$ & $0.05 \pm 0.04$ & 114.09 & $\cdots$ & 0.96 & 7 & 37.2261 & $\mathrm{Y} / \mathrm{Y}$ \\
\hline 32000440 & 005826.41 & $-3400 \quad 56.4$ & 07 Oct2001 & $20.21 \pm 0.08$ & $1.03 \pm 0.10$ & $0.27 \pm 0.13$ & 116.60 & $\cdots$ & 0.42 & 6 & 33.9410 & $\mathrm{Y} / \mathrm{Y}$ \\
\hline 32000568 & 005843.12 & -340304.6 & 13 Nov 2000 & $18.28 \pm 0.04$ & $1.33 \pm 0.05$ & $0.09 \pm 0.05$ & 127.20 & $\cdots$ & 0.50 & 7 & 34.3987 & $\mathrm{Y} / \mathrm{Y}$ \\
\hline 32000683 & 005852.46 & $\begin{array}{lll}-33 & 58 & 08.8\end{array}$ & 07 Oct 2001 & $18.50 \pm 0.04$ & $1.41 \pm 0.05$ & $0.03 \pm 0.05$ & 109.90 & $\cdots$ & 0.64 & 7 & 27.3635 & $\mathrm{Y} / \mathrm{Y}$ \\
\hline 51001133 & $\begin{array}{lll}01 & 07 & 22.41\end{array}$ & -343952.4 & 27Jan2004 & $18.40 \pm 0.03$ & $1.42 \pm 0.03$ & $0.03 \pm 0.03$ & 118.78 & $\cdots$ & 0.76 & 7 & 118.2983 & $\mathrm{Y} / \mathrm{Y}$ \\
\hline 59000195 & 010751.03 & -341931.5 & 31Dec2004 & $19.68 \pm 0.06$ & $1.00 \pm 0.08$ & $0.17 \pm 0.09$ & 70.01 & $\cdots$ & 0.43 & 4 & 107.3482 & $\mathrm{Y} / \mathrm{N}$ \\
\hline
\end{tabular}

${ }^{a}$ Dates for stars multiply observed by us are given in Table 2 .

${ }^{\mathrm{b}}$ Units are $\mathrm{kms}^{-1}$.

${ }^{\mathrm{c}}$ Errors from literature stars; $1 / \sqrt{\sum\left(1 / \epsilon_{v_{i}}^{2}\right)}$ for multiply observed stars. Both are given in km s ${ }^{-1}$. Errors for stars observed by us on Nov 2000, Oct 2001 , Jan 2004, and Dec 2004 are adopted as $10,4,1.5$, and $2.5 \mathrm{~km} \mathrm{~s}^{-1}$, respectively.

dSatisfaction of our photometric criteria (§3) is denoted by a "Y" or "N" for P. Satisfaction of our velocity criteria ( $\$ 4)$ is denoted similarly for V.

${ }^{\text {e }}$ Star selected as giant candidate but fell redward of our RGB/RHB selection at the bright end.

${ }^{\mathrm{f}}$ Previously observed star that is consistent with our photometric candidate constraints but does not satisfy our $\chi$ morphological constraint.

g Photometrically selected BHB candidate.

${ }^{\mathrm{h}} \mathrm{Star}$ fell redward of our BHB selection.

${ }^{\mathrm{i}} \mathrm{Star}$ fell blueward of our 2CD selection limit.

${ }^{\mathrm{j}}$ Mean $v_{\text {hel }}$ inconsistent with Sculptor but at least one individual observation was consistent. 
Table 5. Summary of Photometric Selection Accuracy

\begin{tabular}{lrcc}
\hline \hline & V: Yes & V: No & \% Correct \\
\hline P: Yes (RGB) & 137 & 8 & 94 \\
P: Yes (BHB) & 2 & 0 & 100 \\
P: No & 4 & 6 & 60 \\
P: Yes (Lit) & 9 & 1 & 90 \\
P: No (Lit) & 5 & 7 & 58 \\
\hline P: Yes (Tot) & 148 & 8 & 94 \\
\hline
\end{tabular}

Table 6. Mean Magnitude and Color Errors ${ }^{\mathrm{a}}$

\begin{tabular}{lcrccccc}
\hline \hline \multicolumn{1}{c}{ Sample $^{\mathrm{b}}$} & $N_{\text {fields }}$ & $N_{\text {stars }}$ & $\left\langle\epsilon_{M}\right\rangle$ & $\left\langle\epsilon_{\left(M-T_{2}\right)}\right\rangle$ & $\left\langle\epsilon_{(M-D D O 51)}\right\rangle$ & $\left\langle\epsilon_{V}\right\rangle$ & $\left\langle\epsilon_{(B-V)}\right\rangle$ \\
\hline Error Limit & 96 & 11794 & $0.041 \pm 0.023$ & $0.057 \pm 0.031$ & $0.061 \pm 0.034$ & $\ldots$ & $\ldots$ \\
Giants & 96 & 2018 & $0.051 \pm 0.023$ & $0.067 \pm 0.029$ & $0.073 \pm 0.033$ & $\ldots$ & $\ldots$ \\
RHB $^{\mathrm{c}}$ & $\ldots$ & 386 & $0.104 \pm 0.023$ & $0.129 \pm 0.024$ & $0.156 \pm 0.030^{\mathrm{d}}$ & $0.049 \pm 0.018$ & $0.070 \pm 0.026$ \\
Lower RGB & $\ldots$ & 677 & $0.138 \pm 0.046$ & $0.166 \pm 0.054$ & $0.219 \pm 0.080^{\mathrm{d}}$ & $0.064 \pm 0.028$ & $0.100 \pm 0.044$ \\
BHB & 96 & 1096 & $0.100 \pm 0.036$ & $0.155 \pm 0.048$ & $0.148 \pm 0.052^{\mathrm{d}}$ & $\ldots$ & $\ldots$ \\
$M \leq 19.0$ & 95 & 634 & $0.029 \pm 0.009$ & $0.040 \pm 0.011$ & $0.041 \pm 0.014$ & $\ldots$ & $\ldots$ \\
$M \leq 20.3$ & 80 & 1410 & $0.047 \pm 0.023$ & $0.060 \pm 0.025$ & $0.066 \pm 0.031$ & $\ldots$ & $\ldots$ \\
$M \leq 21.0$ & 53 & 134 & $0.047 \pm 0.017$ & $0.077 \pm 0.026$ & $0.070 \pm 0.024$ & $\ldots$ & $\ldots$ \\
$T_{2} \leq 19.9$ & 70 & 442 & $0.089 \pm 0.028$ & $0.129 \pm 0.033$ & $0.129 \pm 0.041^{\mathrm{d}}$ & $\ldots$ & $\ldots$ \\
$T_{2} \leq 20.3$ & 43 & 853 & $0.097 \pm 0.030$ & $0.147 \pm 0.040$ & $0.143 \pm 0.045^{\mathrm{d}}$ & $\ldots$ & $\ldots$ \\
\hline
\end{tabular}

${ }^{a} \pm$ values are standard deviations.

${ }^{\mathrm{b}}$ Error Limit - Stars satisfying error and morphological limits (§2); Giants - Stars satisfying the 2CD selection; RHB, Lower RGB - Stars selected to be RHB or Lower RGB stars according to Equations 1 and 2; BHB - Full BHB sample selected in Figure 8.

${ }^{c}$ Eight stars in this Majewski et al. (1999) sample did not have measured $M, T_{2}$, and DDO51 magnitudes. ${ }^{\mathrm{d}}$ These values are immaterial because the $(M-D D O 51)$ color was not used in the selection of this sample. 
Table 7. Counts In Offset CMD Selection Boxes

\begin{tabular}{|c|c|c|c|c|c|}
\hline \multirow[b]{2}{*}{$\Delta M$} & \multicolumn{3}{|c|}{$M$ Limit } & \multicolumn{2}{|c|}{$T_{2}$ Limit } \\
\hline & 19.0 & 20.3 & 21.0 & 19.9 & 20.3 \\
\hline 0.00 & 634 & 1410 & 134 & 442 & 853 \\
\hline-0.25 & 485 & 1207 & 111 & 151 & 613 \\
\hline-0.50 & 288 & 945 & 70 & 50 & 215 \\
\hline-0.75 & 120 & 698 & 56 & 18 & 69 \\
\hline-1.00 & 34 & 445 & 42 & 13 & 27 \\
\hline-1.25 & 6 & 219 & 32 & 11 & 19 \\
\hline-1.50 & 6 & 102 & 23 & 10 & 14 \\
\hline-1.75 & 7 & 48 & 20 & 5 & 10 \\
\hline-2.00 & 5 & 20 & 12 & 3 & 6 \\
\hline-2.25 & 4 & 17 & 8 & 2 & 5 \\
\hline-2.50 & 5 & 11 & 9 & 2 & 4 \\
\hline-2.75 & 2 & 12 & 7 & 4 & 3 \\
\hline-3.00 & 1 & 12 & 5 & 4 & 5 \\
\hline-3.25 & $\ldots$ & $\ldots$ & $\ldots$ & 5 & 5 \\
\hline-3.50 & $\ldots$ & $\cdots$ & $\cdots$ & 1 & 4 \\
\hline-3.75 & $\ldots$ & $\ldots$ & $\ldots$ & 1 & 2 \\
\hline-4.00 & $\cdots$ & $\cdots$ & $\ldots$ & 2 & 2 \\
\hline-4.25 & $\ldots$ & $\ldots$ & $\ldots$ & 2 & 1 \\
\hline-4.50 & $\ldots$ & $\ldots$ & $\ldots$ & 1 & 1 \\
\hline-4.75 & $\cdots$ & $\ldots$ & $\ldots$ & 0 & 0 \\
\hline-5.00 & $\ldots$ & $\ldots$ & $\ldots$ & 0 & 0 \\
\hline-5.25 & $\cdots$ & $\cdots$ & $\cdots$ & 1 & 0 \\
\hline-5.50 & $\ldots$ & $\ldots$ & $\ldots$ & 2 & 0 \\
\hline-5.75 & $\ldots$ & $\ldots$ & $\cdots$ & 2 & 0 \\
\hline Area $^{\mathrm{a}}$ & 7.79 & 7.00 & 4.22 & 5.95 & 3.72 \\
\hline$\Sigma_{\mathrm{CMD}}{ }^{\mathrm{b}}$ & 0.16 & 0.57 & 0.48 & 0.10 & 0.18 \\
\hline$\sigma_{\Sigma_{\mathrm{CMD}}} \mathrm{b}$ & \pm 0.08 & \pm 0.15 & \pm 0.18 & \pm 0.06 & \pm 0.11 \\
\hline
\end{tabular}

${ }^{\mathrm{a} U n i t s}$ are square degrees.

${ }^{\mathrm{b}}$ Units are $10^{-3} \operatorname{arcmin}^{-2}$. 
Table 8. Control Field Background Number Densities ${ }^{\mathrm{a}}$

\begin{tabular}{|c|c|c|c|c|c|}
\hline Sample ${ }^{b}$ & $\sum_{\text {North }}$ & $\sum$ South & $\sum_{\text {East }}$ & $\sum$ West & $\sum_{\mathrm{CF}}$ \\
\hline Giants & $5.7_{-5.7}^{+9.3+4.5}$ & $5.7_{-5.7}^{+9.3}+4.5$ & $5.7_{-5.7}^{+9.3+4.5}$ & $\begin{array}{ll}2.8_{-2.8}^{+8.2}+3.7 & -2.8\end{array}$ & $5.0_{-2.6}^{+4.5}+2.2$ \\
\hline$M \leq 19.0$ & $0.0_{-0.0}^{+6.5}+3.1$ & $0.0_{-0.0}^{+6.5}+3.1$ & $0.0_{-0.0}^{+6.5}+3.1$ & $0.0_{-0.0}^{+6.5}+3.1$ & $0.0_{-0.0}^{+3.6}+1.6$ \\
\hline$M \leq 20.3$ & $0.0_{-0.0}^{+6.5}+0.1$ & $\begin{array}{ll} & +8.2 \\
+8.8 & +3.7 \\
-2.8\end{array}$ & $0.0_{-0.5}^{+6.0}+3.0$ & $0.0+6.5+3.1$ & $0.7_{-0.5}^{+3.0}+1.6$ \\
\hline$M \leq 21.0$ & $2.8_{-2.8}^{+8.2}+3.7$ & $2.8_{-2.8}^{+8.2}+3.8$ & $0.0_{-0.0}^{+6.5}+0.1$ & $\begin{array}{l}-0.0-0.0 \\
\ldots\end{array}$ & $1.9_{-1.3}^{+4.4}+2.0$ \\
\hline$T_{2} \leq 19.9$ & $\ldots$ & $\begin{array}{l}-2.0-2.0 \\
\ldots\end{array}$ & $0.0_{-0.0}^{+6.5}+3.1$ & $\ldots$ & $0.0_{-0.0}^{+6.5}+3.1$ \\
\hline$T_{2} \leq 20.3$ & $\cdots$ & $\cdots$ & $0.0_{-0.0}^{+6.5}+0.0$ & $\cdots$ & $0.0_{-0.0}^{+6.5}+3.1$ \\
\hline
\end{tabular}

${ }^{\text {a } U n i t s ~ a r e ~} 10^{-3} \operatorname{arcmin}^{-2}$. First and second error pairs give, respectively, the $90 \%$ and $68 \%$ Poissonian confidence limits.

${ }^{\mathrm{b}}$ See the text for definitions of the samples.

Table 9. King Radial Profile Fits

\begin{tabular}{|c|c|c|c|c|c|c|c|}
\hline \multirow[b]{2}{*}{ Sample $^{\mathrm{a}}$} & \multicolumn{2}{|c|}{ Center Coords (J2000.0) } & \multirow[b]{2}{*}{$P A\left(^{\circ}\right)$} & \multirow[b]{2}{*}{$\varepsilon$} & \multirow[b]{2}{*}{$r_{c}\left({ }^{\prime}\right)$} & \multirow[b]{2}{*}{$r_{\lim }\left({ }^{\prime}\right)^{\mathrm{b}}$} & \multirow[b]{2}{*}{$\Sigma_{b}^{\mathrm{c}}$} \\
\hline & $\alpha$ & $\delta$ & & & & & \\
\hline$M \leq 19.0$ & $1.001727 \pm 0.000006$ & $-33.71026 \pm 0.00081$ & $95.5 \pm 2.7$ & $0.33 \pm 0.03$ & $6.97 \pm 0.69$ & $85.14 \pm 7.40$ & $0.26 \pm 0.21$ \\
\hline$M \leq 20.3$ & $1.002043 \pm 0.000039$ & $-33.70979 \pm 0.00146$ & $92.4 \pm 1.8$ & $0.28 \pm 0.03$ & $6.50 \pm 0.46$ & $80.76 \pm 5.98$ & $2.95 \pm 0.54$ \\
\hline$T_{2} \leq 19.9$ & $0.999622 \pm 0.000052$ & $-33.71548 \pm 0.00214$ & $96.8 \pm 4.1$ & $0.31 \pm 0.05$ & $8.47 \pm 1.06$ & $81.53 \pm 10.19$ & $1.22 \pm 0.38$ \\
\hline$T_{2} \leq 20.3$ & $1.000712 \pm 0.000028$ & $-33.71270 \pm 0.00308$ & $94.3 \pm 3.1$ & $0.26 \pm 0.04$ & $8.53 \pm 0.78$ & $75.06 \pm 5.46$ & $1.56 \pm 0.54$ \\
\hline Mean ${ }^{\mathrm{d}}$ & $1.001663 \pm 0.000006$ & $-33.71077 \pm 0.00066$ & $93.8 \pm 1.3$ & $0.29 \pm 0.02$ & $7.14 \pm 0.33$ & $79.59 \pm 3.34$ & $\cdots$ \\
\hline 1 & 1.002486 & -33.69407 & $\cdots$ & $>0$ & $\cdots$ & $(80-120)$ & $\cdots$ \\
\hline 2 & 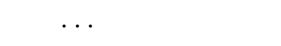 & $\ldots$ & $98 \pm 2$ & $0.35 \pm 0.05$ & $\cdots$ & $46 \pm 3$ & $\cdots$ \\
\hline 3 & 1.019497 & -33.56441 & $\ldots$ & $<0.3$ & 7.5 & $>75$ & $\cdots$ \\
\hline 4 & 1.003975 & -33.69662 & $95.4 \pm 4.3$ & $0.34 \pm 0.04$ & $8.9 \pm 0.9$ & \pm 10 & $\cdots$ \\
\hline 5 & 1.001206 & -33.71407 & $99 \pm 1$ & $0.32 \pm 0.03$ & $5.8 \pm 1.6$ & $76.5 \pm 5.0$ & $\ldots$ \\
\hline 6 & 1.007778 & -33.70000 & $98 \pm 2$ & $0.20 \pm 0.05$ & $7.56 \pm 0.7$ & $40 \pm 4$ & $\cdots$ \\
\hline
\end{tabular}

aReferences: (1) Shapley (1938a,b); (2) H61; (3) Demers, Kunkel \& Krauter (1980); (4) Eskridge (1988b); (5) IH95; (6) W03.

${ }^{\mathrm{b}}$ This parameter is described as the tidal radius, $r_{t}$, in most treatments, including the original discussion by King (1962). We have adopted the name limiting radius, $r_{\mathrm{lim}}$, here to avoid confusion between this fitting parameter and the true tidal radius of the system.

${ }^{\mathrm{c}}$ Units are $10^{-3} \operatorname{arcmin}^{-2}$.

${ }^{\mathrm{d}}$ Error weighted mean of the four samples. Errors will be slightly under-estimated since the samples are not completely independent. 
Table 10. PLC Radial Profile Fits

\begin{tabular}{|c|c|c|c|c|c|c|c|}
\hline \multirow[b]{2}{*}{ Sample } & \multicolumn{2}{|c|}{ Center Coords (J2000.0) } & \multirow[b]{2}{*}{$P A\left(^{\circ}\right)$} & \multirow[b]{2}{*}{$\varepsilon$} & \multirow[b]{2}{*}{$r_{c}\left({ }^{\prime}\right)$} & \multirow[b]{2}{*}{$\nu$} & \multirow[b]{2}{*}{$\Sigma_{b}{ }^{\mathrm{a}}$} \\
\hline & $\alpha$ & $\delta$ & & & & & \\
\hline$M \leq 19.0$ & $1.001751 \pm 0.000008$ & $-33.70764 \pm 0.00047$ & $89.81 \pm 0.12$ & $0.33 \pm 0.04$ & $10.73 \pm 1.03$ & $2.03 \pm 0.13$ & $0.00 \pm 0.04^{\mathrm{b}}$ \\
\hline$M \leq 20.3$ & $1.002271 \pm 0.000005$ & $-33.71114 \pm 0.00050$ & $89.43 \pm 0.38$ & $0.27 \pm 0.03$ & $8.60 \pm 0.69$ & $1.74 \pm 0.10$ & $0.42 \pm 1.71$ \\
\hline$T_{2} \leq 19.9$ & $0.991578 \pm 0.000015$ & $-33.71696 \pm 0.00205$ & $89.90 \pm 0.08$ & $0.49 \pm 0.03$ & $19.55 \pm 3.39$ & $2.82 \pm 0.55$ & $0.80 \pm 0.59$ \\
\hline$T_{2} \leq 20.3$ & $1.001197 \pm 0.000004$ & $-33.71297 \pm 0.00069$ & $89.90 \pm 0.03$ & $0.23 \pm 0.04$ & $11.77 \pm 1.41$ & $1.99 \pm 0.19$ & $0.00 \pm 0.34^{\mathrm{c}}$ \\
\hline Mean $^{d}$ & $1.001299 \pm 0.000003$ & $-33.71016 \pm 0.00030$ & $89.89 \pm 0.03$ & $0.34 \pm 0.02$ & $11.73 \pm 0.50$ & $1.88 \pm 0.07$ & $\cdots$ \\
\hline
\end{tabular}

${ }^{\text {a } U n i t s ~ a r e ~} 10^{-3} \operatorname{arcmin}^{-2}$.

balue: $\Sigma_{b}=2.19 \times 10^{-8} \operatorname{arcmin}^{-2}$.

'Value: $\Sigma_{b}=2.70 \times 10^{-6} \operatorname{arcmin}^{-2}$.

${ }^{\mathrm{d}}$ Error weighted mean of the four samples. Errors will be slightly under-estimated since the samples are not completely independent.

Table 11. Goodness-of-fit Estimates $\left(\chi^{2}\right)$

\begin{tabular}{|c|c|c|c|c|c|c|c|c|}
\hline \multirow[b]{2}{*}{ Sample } & \multicolumn{2}{|c|}{ Model $\Sigma_{b}$} & \multicolumn{6}{|c|}{ Measured $\Sigma_{b}$} \\
\hline & King & PLC & King & PLC & $\mathrm{K}+\mathrm{B}^{\mathrm{a}}$ & $r_{\text {break }}^{a, b}$ & $\mathrm{~K}+\mathrm{B}^{\mathrm{c}}$ & $r_{\text {break }} \mathrm{c}, b$ \\
\hline$M \leq 19.0$ & 4.38 & 4.05 & 4.42 & 4.37 & 3.98 & 61 & 4.26 & 79 \\
\hline$M \leq 20.3$ & 8.77 & 5.67 & 9.58 & 5.54 & 7.81 & 61 & 7.74 & 61 \\
\hline$T_{2} \leq 19.9$ & 3.46 & 5.03 & 5.05 & 5.97 & 2.70 & 57 & 2.71 & 59 \\
\hline$T_{2} \leq 20.3$ & 5.06 & 4.06 & 5.64 & 3.96 & 4.36 & 55 & 4.46 & 60 \\
\hline
\end{tabular}

a "King+Break" profile using the least-squares fit power law to the density points beyond $r_{\text {break }}$ as given in Figure 16. The least-squares fit for the $M \leq 20.3$ did not include the final point (because it was below the background) so the $\Sigma \propto r^{-1.9}$ fit is actually worse than the $\Sigma \propto r^{-2}$ fit.

${ }^{\mathrm{b}}$ Semi-major axis distance given in arcmin.

c "King +Break" profile using a $\Sigma \propto r^{-2}$ power law for the break population.

Table 12. Kolmogorov-Smirnov Statistics for Split RGB Populations

\begin{tabular}{lccc}
\hline \hline \multicolumn{1}{c}{ Comparison } & $N_{e}$ & $D$ & KS\% \\
\hline$M \leq 19.0$ blue to red & 157.6656 & 0.2491 & $4.2483 \times 10^{-7}$ \\
$M \leq 20.3$ blue to red & 364.2317 & 0.1562 & $3.0136 \times 10^{-6}$ \\
$T_{2} \leq 20.3$ to $M \leq 20.3$ blue & 339.8766 & 0.0233 & 99.196 \\
$T_{2} \leq 20.3$ to $M \leq 20.3$ red & 360.7070 & 0.1522 & $8.9081 \times 10^{-6}$ \\
\hline
\end{tabular}


Table 13. King Radial Profile Fits for Split RGB Populations

\begin{tabular}{|c|c|c|c|c|c|c|c|}
\hline \multirow[b]{2}{*}{ Sample } & \multicolumn{2}{|c|}{ Center Coords (J2000.0) } & \multirow[b]{2}{*}{$P A\left(^{\circ}\right)$} & \multirow[b]{2}{*}{$\varepsilon$} & \multirow[b]{2}{*}{$r_{c}\left({ }^{\prime}\right)$} & \multirow[b]{2}{*}{$r_{\lim }\left({ }^{\prime}\right)$} & \multirow[b]{2}{*}{$\Sigma_{\mathrm{CMD}}{ }^{\mathrm{a}}$} \\
\hline & $\alpha$ & $\delta$ & & & & & \\
\hline Blue $M \leq 19.0$ & $1.002102 \pm 0.000011$ & $-33.70122 \pm 0.00131$ & $96.3 \pm 4.7$ & $0.27 \pm 0.05$ & $9.57 \pm 1.31$ & $89.24 \pm 10.78$ & $0.12 \pm 0.07$ \\
\hline $\operatorname{Red} M \leq 19.0$ & $1.001609 \pm 0.000007$ & $-33.71245 \pm 0.00091$ & $96.0 \pm 3.1$ & $0.41 \pm 0.04$ & $6.08 \pm 0.86$ & $65.74 \pm 7.00$ & $0.05 \pm 0.04$ \\
\hline Blue $M \leq 20.3$ & $1.002319 \pm 0.000048$ & $-33.70173 \pm 0.00256$ & $92.6 \pm 2.1$ & $0.25 \pm 0.04$ & $7.47 \pm 0.78$ & $92.63 \pm 9.96$ & $0.44 \pm 0.13$ \\
\hline $\operatorname{Red} M \leq 20.3$ & $1.002213 \pm 0.000032$ & $-33.71280 \pm 0.00219$ & $96.9 \pm 3.0$ & $0.34 \pm 0.03$ & $5.95 \pm 0.59$ & $71.28 \pm 5.48$ & $0.26 \pm 0.10$ \\
\hline
\end{tabular}

aUnits are $10^{-3} \operatorname{arcmin}^{-2}$ as derived from the "CMD offset" method.

Table 14. Fractional Mass Loss Estimates ${ }^{\mathrm{a}}$

\begin{tabular}{lrcrrrr}
\hline \hline Sample & $n_{\text {break }}{ }^{\mathrm{b}}$ & $\Sigma_{\text {break }}{ }^{\mathrm{c}}$ & $r_{x t}\left({ }^{\prime}\right)$ & $n_{x t} \mathrm{~b}$ & $\left(\frac{d f}{d t}\right)_{1} \mathrm{~d}$ & $\left(\frac{d f}{d t}\right)_{2} \mathrm{~d}$ \\
\hline$M \leq 19.0$ & 623.7 & 1.97 & 80.4 & 5.3 & 3.97 & 4.41 \\
$M \leq 20.3$ & 1350.6 & 7.63 & 104.5 & 37.5 & 5.62 & 7.89 \\
$T_{2} \leq 19.9$ & 422.4 & 3.61 & 135.8 & 17.6 & 4.84 & 11.95 \\
$T_{2} \leq 20.3$ & 839.1 & 5.27 & 104.5 & 10.1 & 2.44 & 8.77 \\
Mean & \multicolumn{1}{c}{$\cdots$} & $\cdots$ & $\cdots$ & $\cdots$ & 4.22 & 8.26 \\
\hline
\end{tabular}

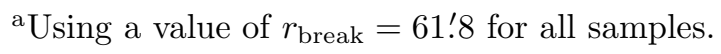

${ }^{\mathrm{b}}$ Non-integer values due to background subtraction.

${ }^{\mathrm{c}}$ Units are $10^{-3} \operatorname{arcmin}^{-2}$.

${ }^{\mathrm{d}}$ Units are $10^{-2} \mathrm{Gyr}^{-1}$. 

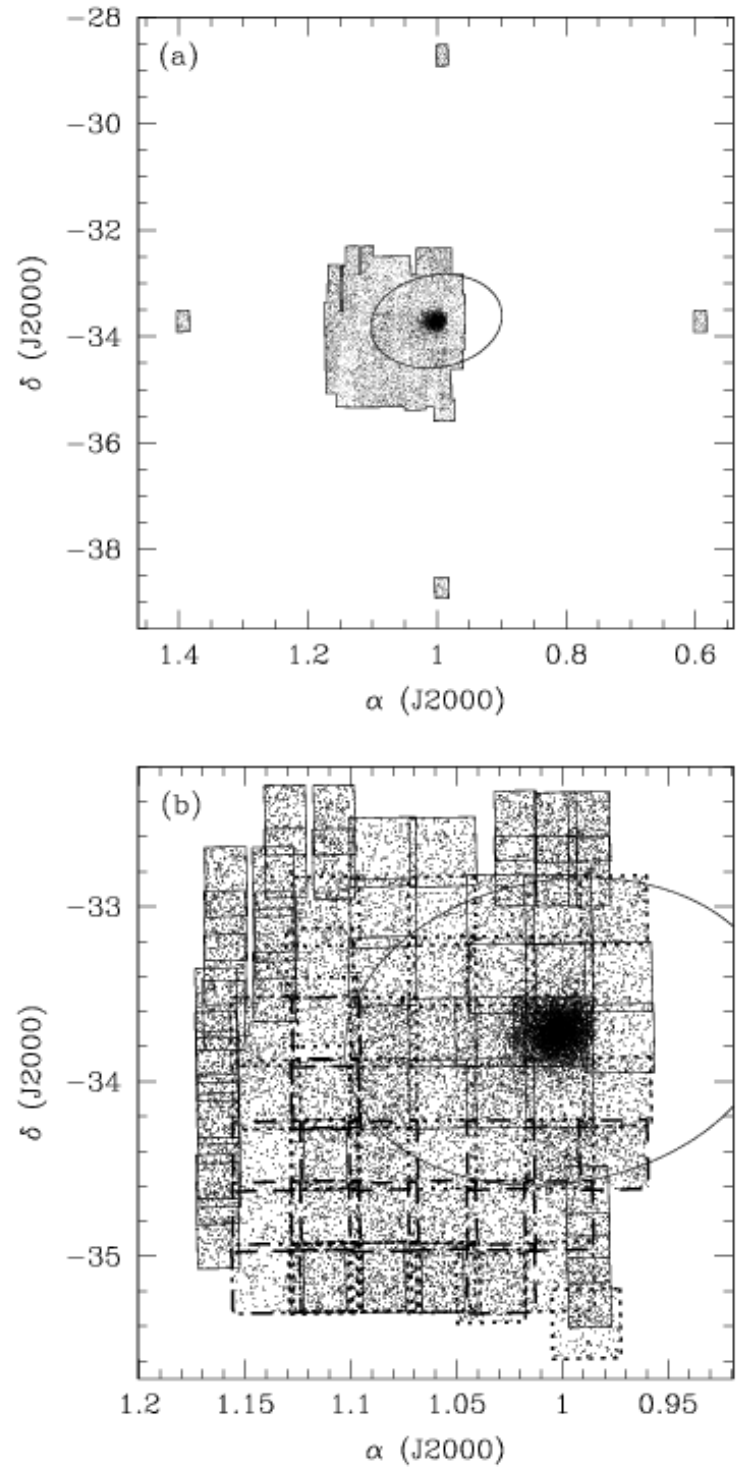

Fig. 1. - Spatial distribution of field boundaries and all photometered objects over (a) the entire survey area and (b) the contiguous inner region. The ellipse centered on the core concentration of the $\mathrm{dSph}$ is the King limiting radius, $r_{\lim }=76.5$, derived by IH95. The survey area extends to $\sim 2 r_{\text {lim }}$ from the center to the south and east. In (a), only the outer boundary of the inner contiguous fields is shown. In (b), individual pointing boundaries are shown with those taken under photometric conditions (54 out of 96) using solid boundaries, and fields requiring one (27), two (12), and three (3) boot-strapping steps from a photometric frame (explained in text) using dotted, dashed, and dot-dashed boundaries, respectively. The varying depth of the fields (see Figure 4) is due to differences in observing conditions. 


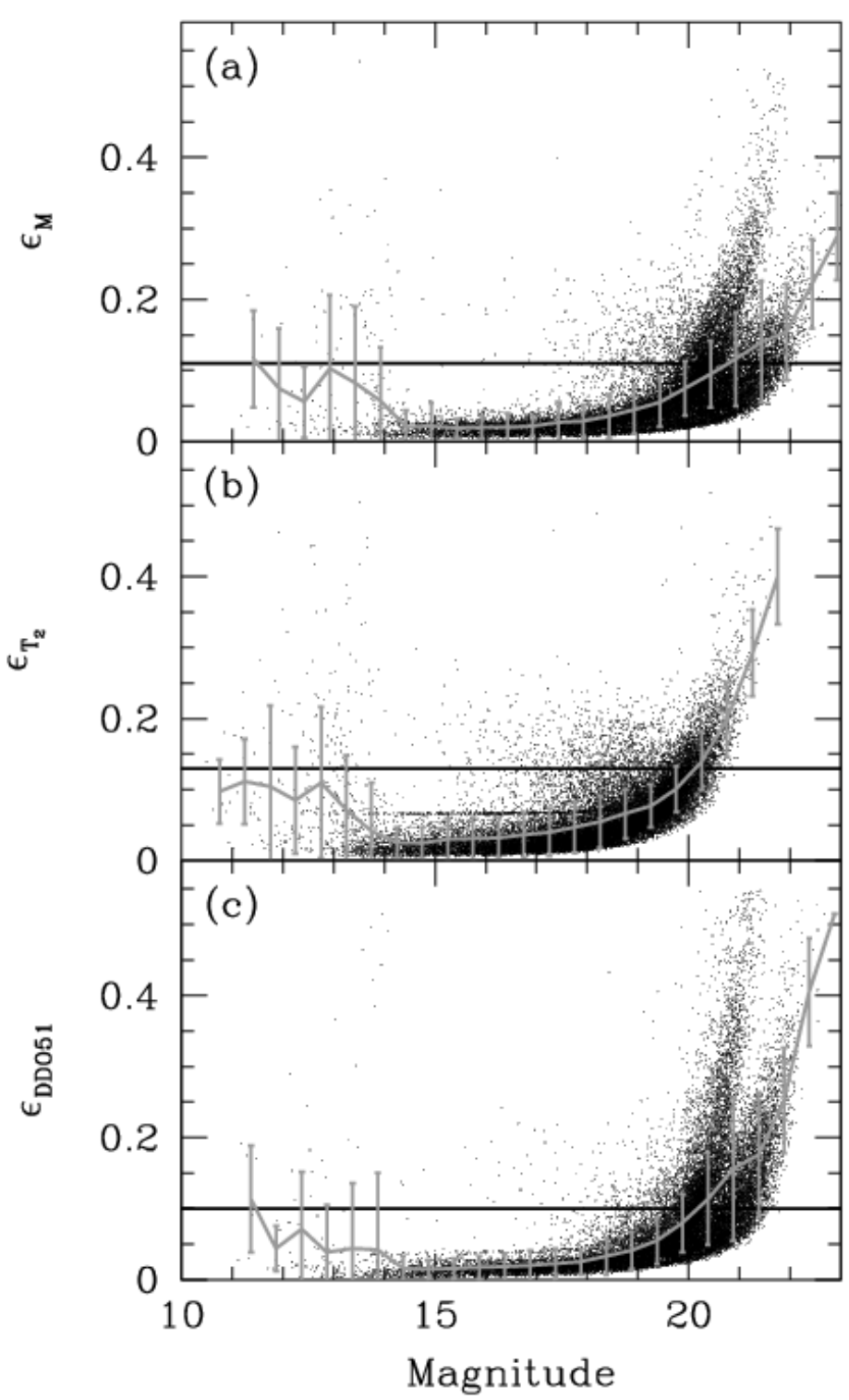

Fig. 2.- PSF measures of the magnitude error, $\epsilon_{X}$, with respect to the determined extinction corrected magnitude for the (a) $X=M$, (b) $X=T_{2}$, and (c) $X=D D O 51$ filters. The mean (grey line) and standard deviation (error bars) are shown to give a better sense of the statistical distribution of points. The solid black lines of constant $\epsilon_{X}$ denote the adopted error limits. Stars with measurement errors larger than these limits are discarded from any analysis. Note that some pointings have photometry for which $\epsilon_{X}$ does not asymptote to zero. This is most noticeable in the $T_{2}$ filter and is a product of a poorly converging PSF for some frames. 


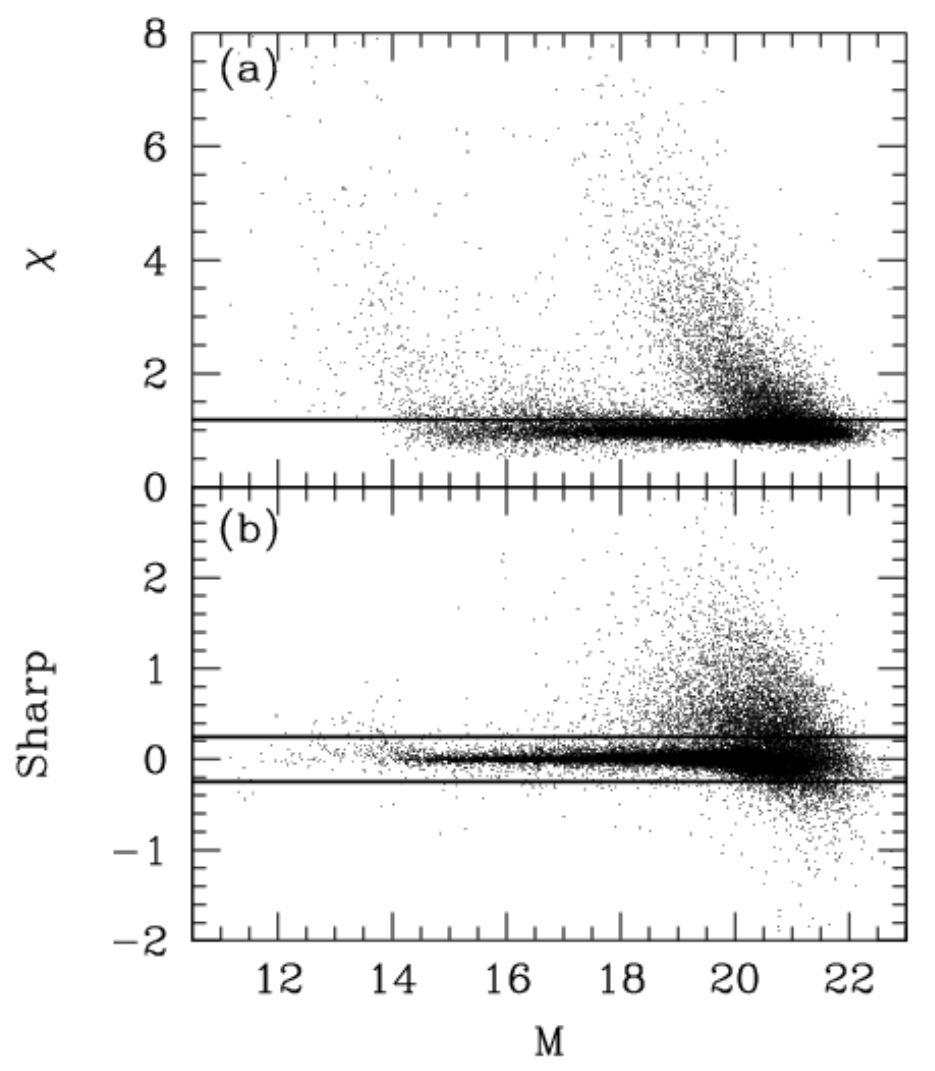

Fig. 3. - The PSF fitting parameters (a) $\chi$ and (b) sharp are plotted versus $M$ to show the stellar loci of good photometric measures. The measured $\chi$ and sharp values for each CCD frame have been offset so that the stellar loci lie along $\chi=1$ and sharp $=0$. The solid lines denote the adopted range of acceptable "stellar" morphologies of these normalized parameters: (a) $\chi<1.18$ and (b) $-0.25<\operatorname{sharp}<0.25$. 

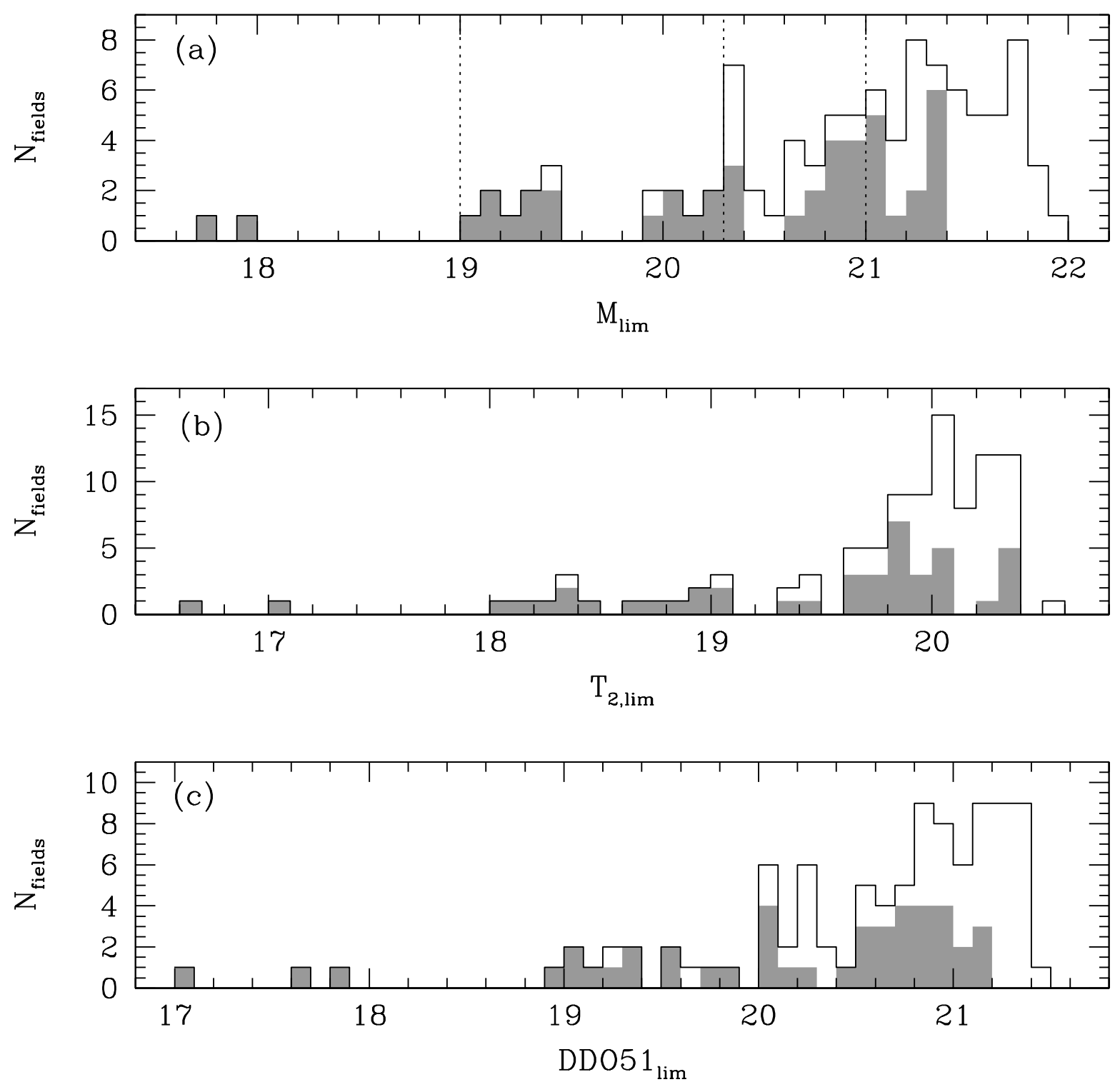

Fig. 4.- A histogram of the limiting magnitudes of all the pointings in the survey for (a) $M$, (b) $T_{2}$ and (c) DDO51. The grey regions give the number of non-photometric fields in each bin. Magnitude limits are determined by the mean magnitude of objects near the error limits of each frame. The magnitude limits that will define some of our analysis samples (§3.3) are shown by vertical dotted lines in the $M$ diagram. 


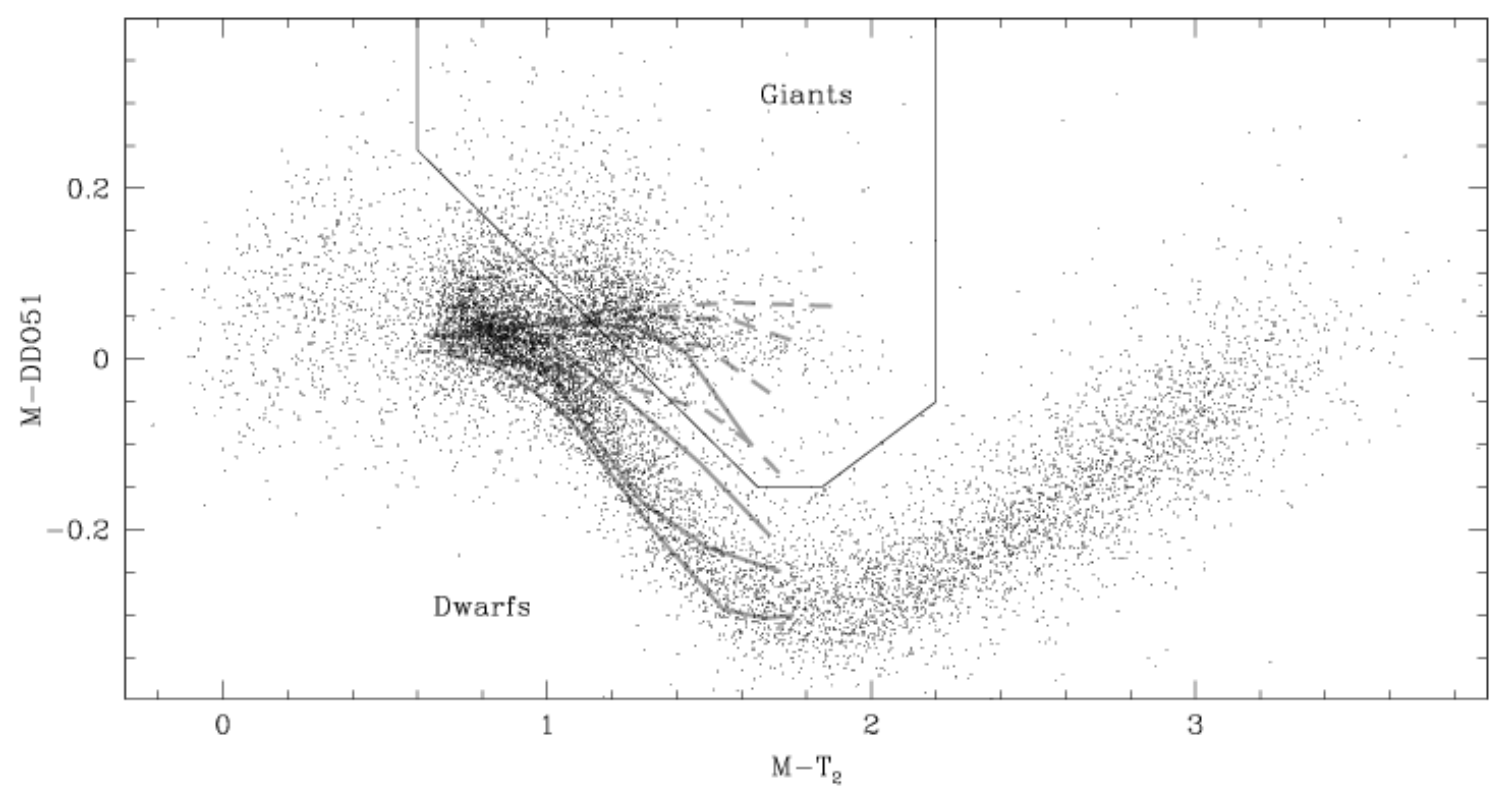

Fig. 5.- The two-color diagram (2CD) of all stellar objects in our survey that fall within the magnitude and profile shape limits described in $\S 2$. The limits of our 2CD selection of giants are plotted as solid straight black lines; the limits were selected to lie along, but above the dwarf 2CD locus. Isometallicity lines for dwarfs (solid grey lines) and for giants (dashed grey lines) are plotted for reference (from Figure 2b of Paper I). In increasing ( $M-D D O 51)$ color, these lines correspond to $[\mathrm{Fe} / \mathrm{H}]=0.0,-1.0,-2.0,-3.0$ for dwarfs and $[\mathrm{Fe} / \mathrm{H}]=$ $-1.0,-1.5,-2.0,-3.0$ for giants. 


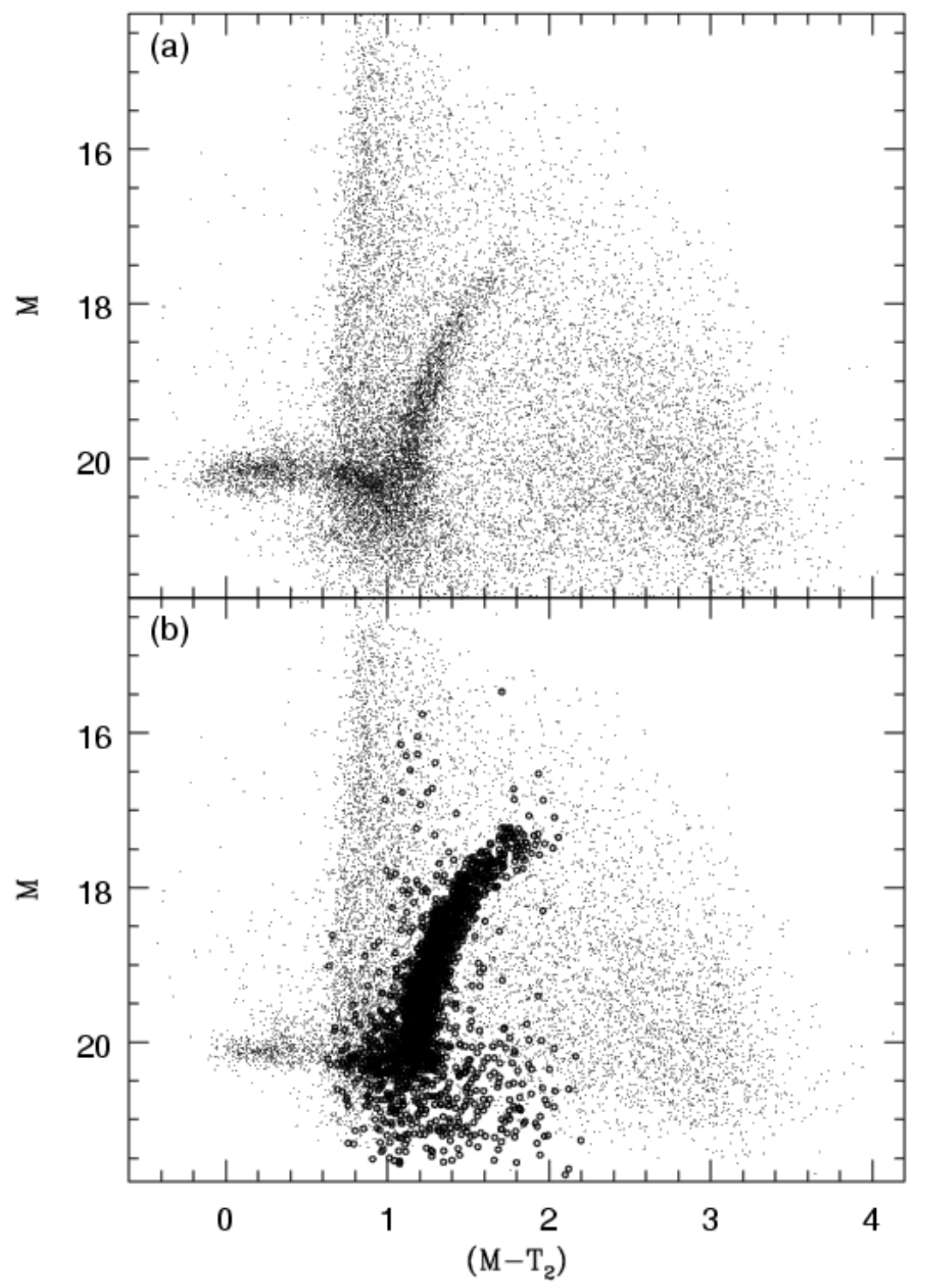

Fig. 6. - The color-magnitude diagrams (CMDs) of (a) all the star-like objects (as determined by PSF-fitting parameters) in our survey and (b) those objects with magnitude errors within the limits defined in $\S 2$. Also, in (b), those stars selected to be giant star candidates in the 2CD (Figure 5) are plotted as open circles. 


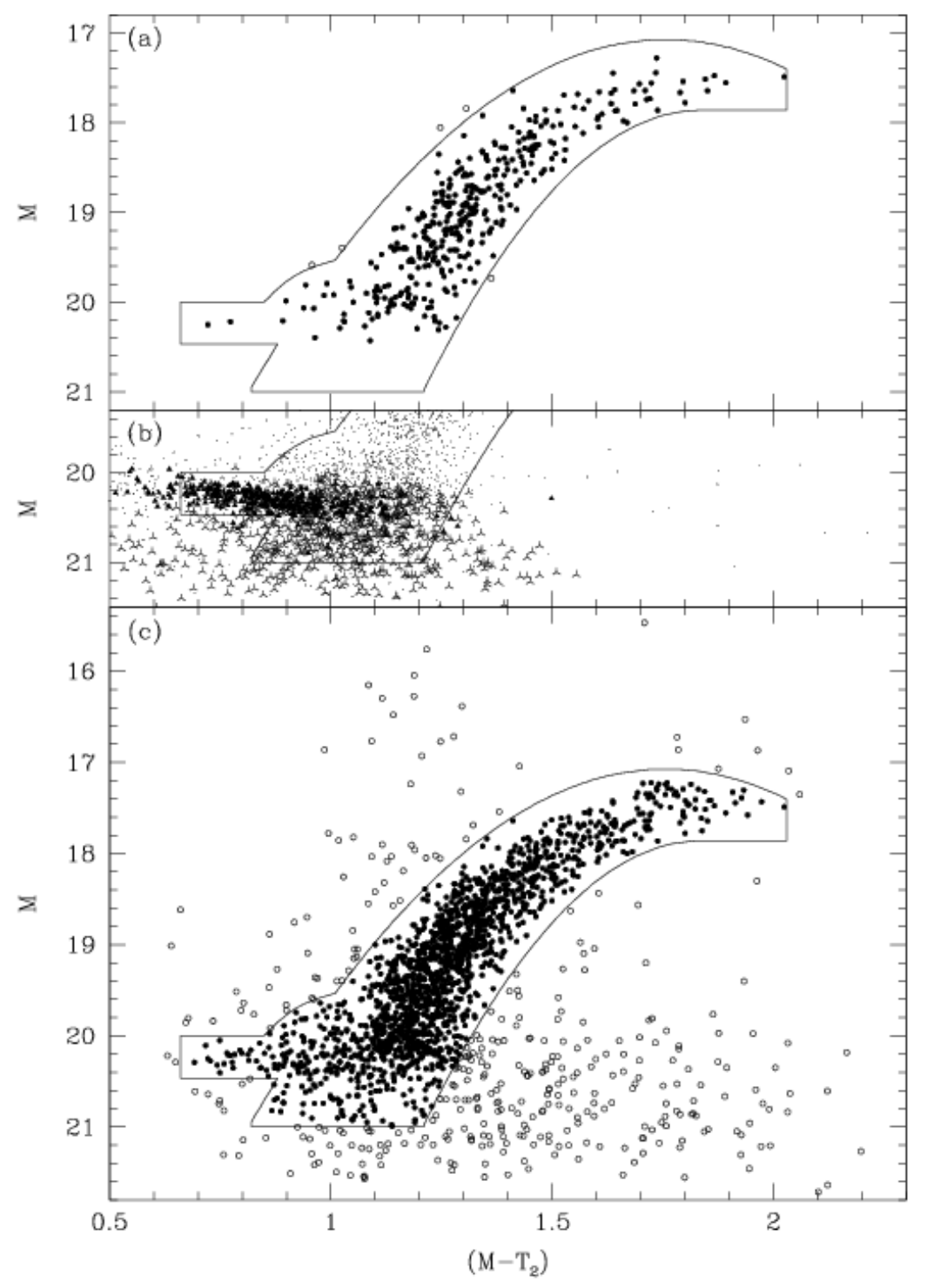

Fig. 7.- The CMD of stars selected as giant star candidates. Panel (a) shows the CMD of the giants found to have Sculptor proper motion membership probabilities greater than $80 \%$ by Schweitzer et al. (1995). These stars are used to define the RGB/RHB selection to $M \sim 20.5$. As described more fully in the text, (b) displays the RHB stars (triangles) and the lower portion of the RGB (three-pointed crosses) as selected from the $(B-V, V)$ CMD in Majewski et al. (1999). All stars photometered both in our study and in Majewski et al. (1999) (points) are shown in (b). This plot was used to define the RGB/RHB selection to $M \sim 21.0$. Finally, in (c), we demonstrate the application of our RGB/RHB selection to all giant star candidates found in our survey. In (a) and (c), those giant candidates falling inside our RGB/RHB selection are represented by filled circles, while those outside are left as open circles. 


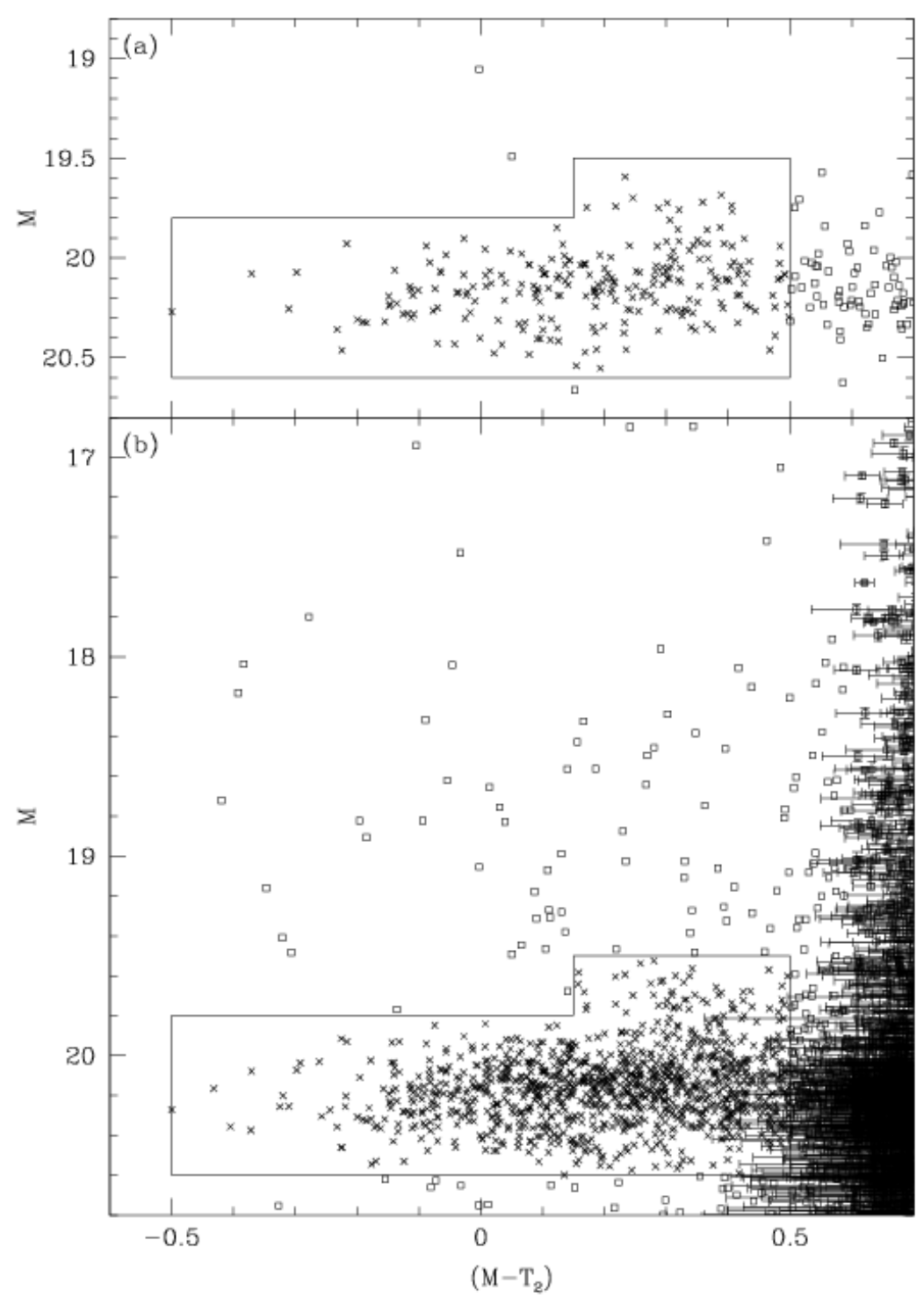

Fig. 8. - The CMD of stars in the color range of the BHB of Sculptor. Both (a) and (b) plot all stellar objects regardless of magnitude errors. The definition of the BHB selection is done in (a), which shows only those stars with Sculptor proper motion membership probabilities greater than $80 \%$. In (b), we demonstrate the application of this selection to all the stellar objects in our survey. In both plots, those objects selected to be Sculptor BHB candidates are shown as crosses and those left unselected are open squares. Finally, (b) displays error bars in both color and magnitude for objects with $\left(M-T_{2}\right)>0.6$ (the approximate blue edge of the MW field population) to demonstrate that there is little likely dwarf star contamination in this sample. 

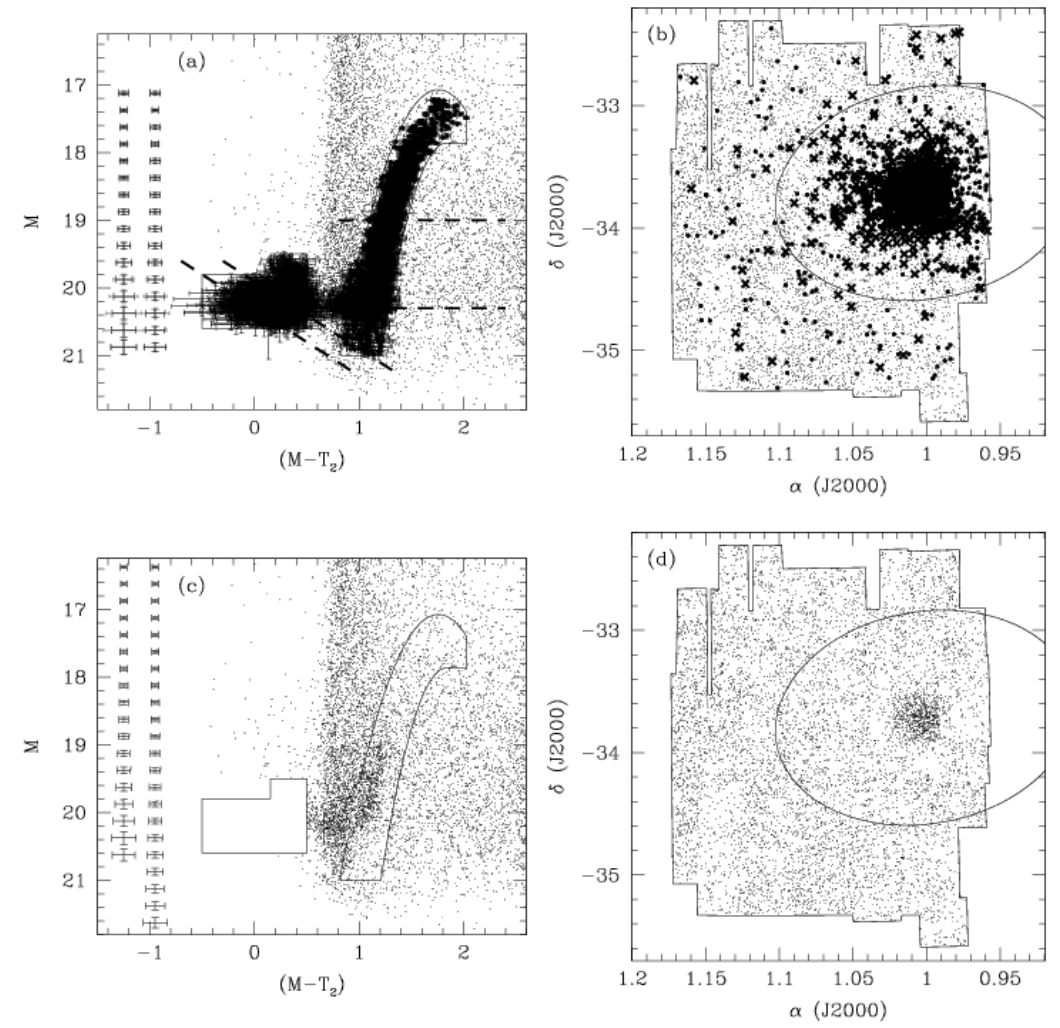

Fig. 9. - The distribution of all stars from our magnitude error and morphology-limited detected sample in the CMD (left panels) and on the sky (right panels). The top panels include the stars that meet our Sculptor candidate criteria shown with larger symbols (filled circles for RGB/RHB and crosses for BHB, including individual error bars) while the bottom panels show only those objects that were eliminated by our selection criteria. The boundaries of our CMD selection are shown in panels (a) and (c). Dashed lines in panel (a) show the magnitude limits adopted in our analysis of the structure of the dSph. Also, we show two sets of average color and magnitude errors in 0.25 magnitude bins at the left of panels (a) and (c). The set to the left is for stars at a radius of $\leq 0.5 r_{l i m}$, while the one to the right is for stars beyond this radius. The average errors are calculated for the Sculptor candidates in (a) and for all de-selected, field stars in (c). Average error bars are determined both with (grey) and without (black) the BHB population in (a). Panel (b) demonstrates the general correspondence of the BHB and RGB/RHB distributions as well as the significant number of stars outside the nominal limiting radius determined by IH95 (plotted ellipse in panels (b) and $(\mathrm{d})$ ). By comparison with Figure 1 it may be seen that some of the outermost Sculptor candidates appear to lie along individual CCD field boundaries (where the net depth of the survey is deeper). We account for this effect in our analysis by choosing magnitude limited samples. Our conservative Sculptor selection criteria have actually de-selected some likely Sculptor stars, as evidenced by the higher density of stars near the bottom of the RGB in panel (c) and the concentration of stars at the spatial center of Sculptor in panel (d). 

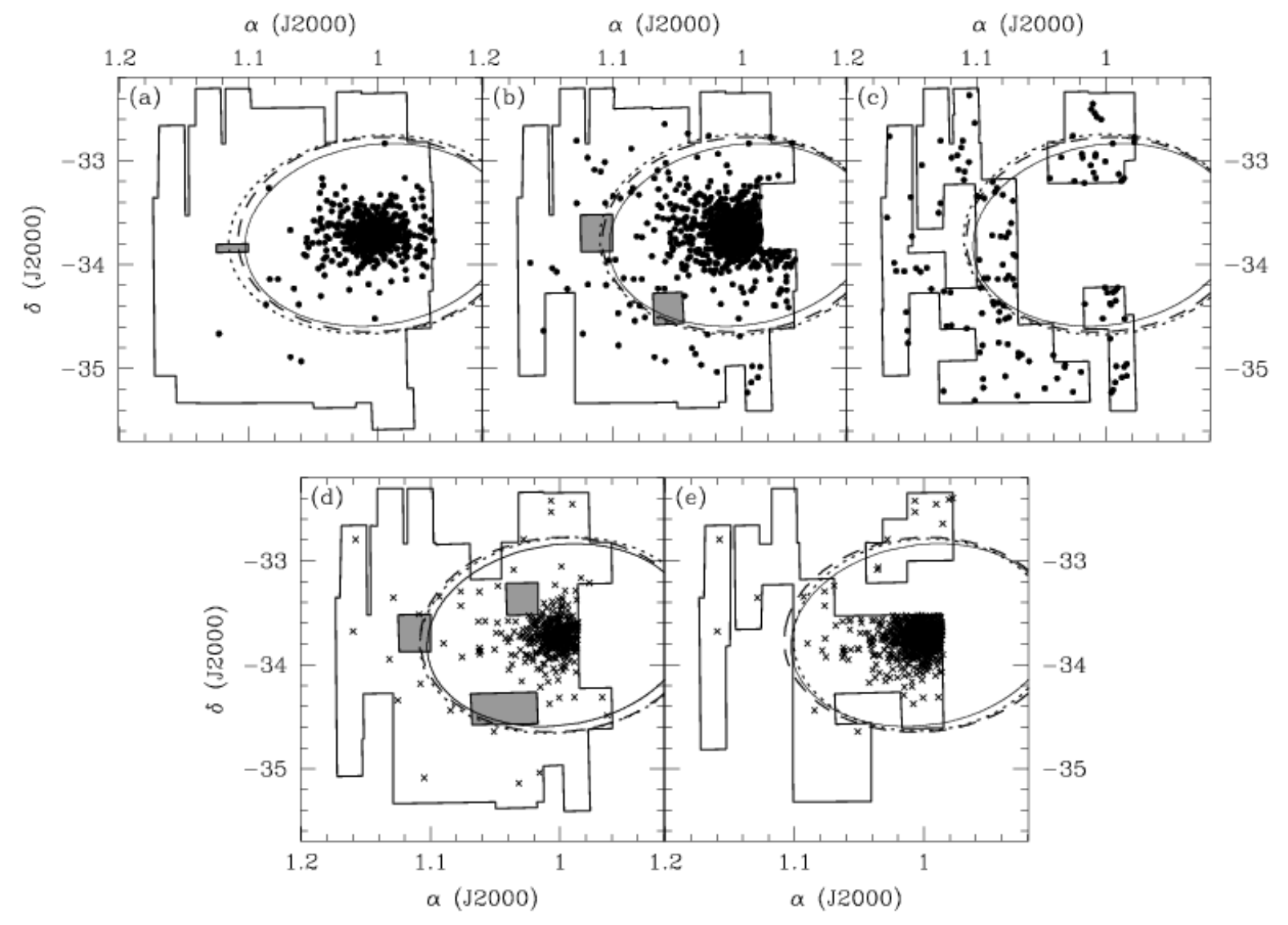

Fig. 10. - The spatial plots of all magnitude limited samples and their respective field boundaries or survey holes (grey): (a) $M \leq 19.0$, (b) $M \leq 20.3$, (c) $M \leq 21.0$, (d) $T_{2} \leq 19.9$, and (e) $T_{2} \leq 20.3$. Each panel gives for its subsample the limiting radius corresponding to the best-fit King profile (dotted ellipse; see Table 9), the limiting radius corresponding to the mean King profile parameters across all subsamples (Table 9; dashed ellipse), and the IH95 (solid ellipse) King limiting radius (§5.2). The ellipses in (c) are the same as those in (b) because the $M \leq 21.0$ sample was not fit to a radial profile (see text). The four derived King limiting radii (for the $M \leq 19.0, M \leq 20.3, T_{2} \leq 19.9$, and $T_{2} \leq 20.3$ samples) are only slightly different from one another and the same within the fitting errors. 

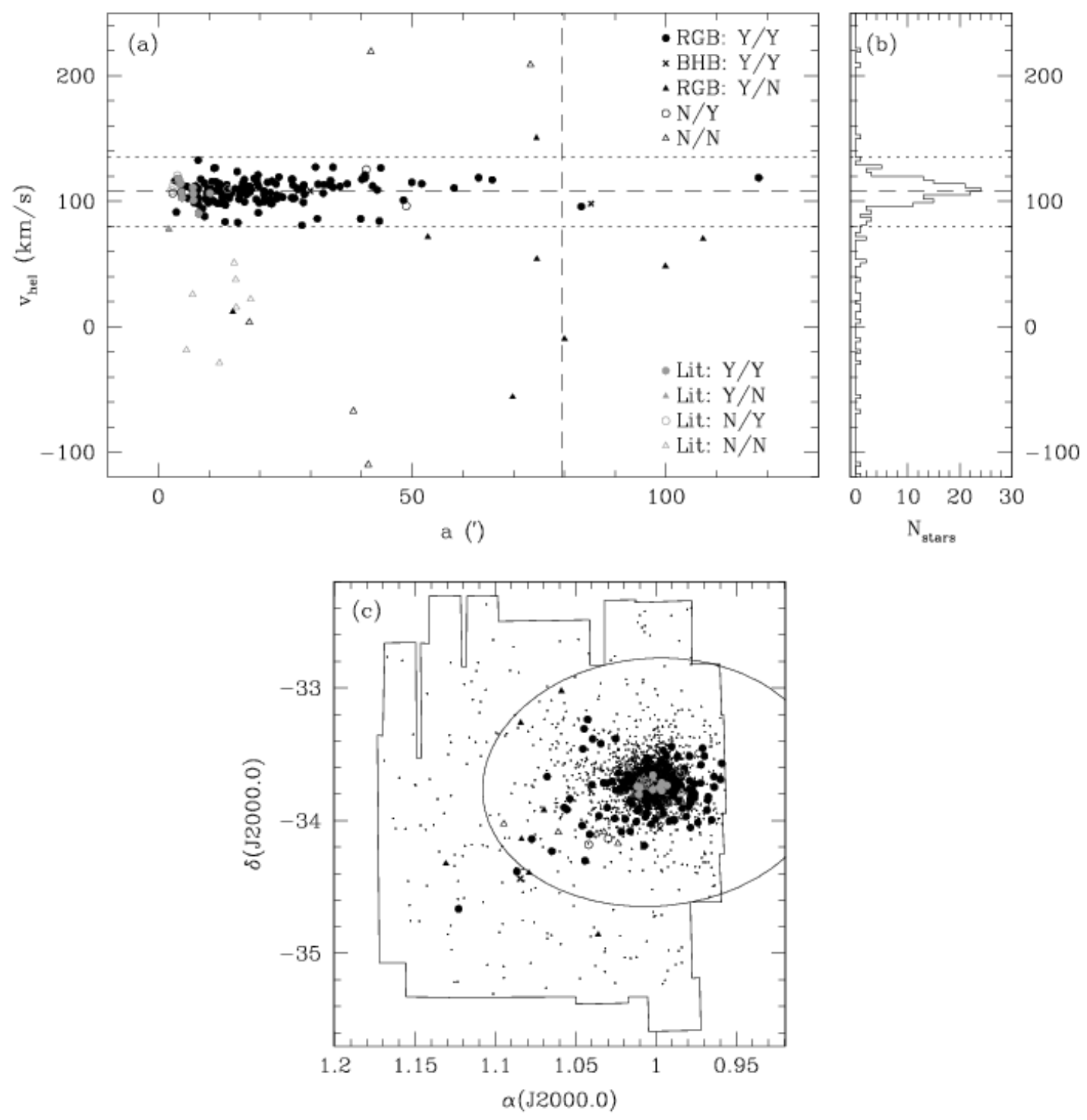

Fig. 11. - The (a) heliocentric radial velocity as a function of elliptical distance, $a$, from the derived cluster center, (b) histogram of the number of stars, $N_{\text {stars }}$, at each velocity, and (c) spatial distribution of spectroscopically observed stars. The legend in (a) gives the definition of each point symbol according to stellar type and photometric/velocity member status. The distribution of points within $80 \leq v_{\text {hel }} \leq 135$ (within the horizontal dotted lines) is roughly symmetric about the derived $\overline{v_{\text {hel }}}=107.96 \pm 0.76$ (horizontal dashed line). The small points in (c) are stars photometrically selected to be Sculptor candidates (BHB and RGB/RHB) but have not yet been observed spectroscopically. In particular, note (a) and (c) show six stars observed outside our derived mean King limiting radius (a: vertical dashed line, c: solid ellipse). Three of these stars are both photometrically and spectroscopically selected as Sculptor members. 


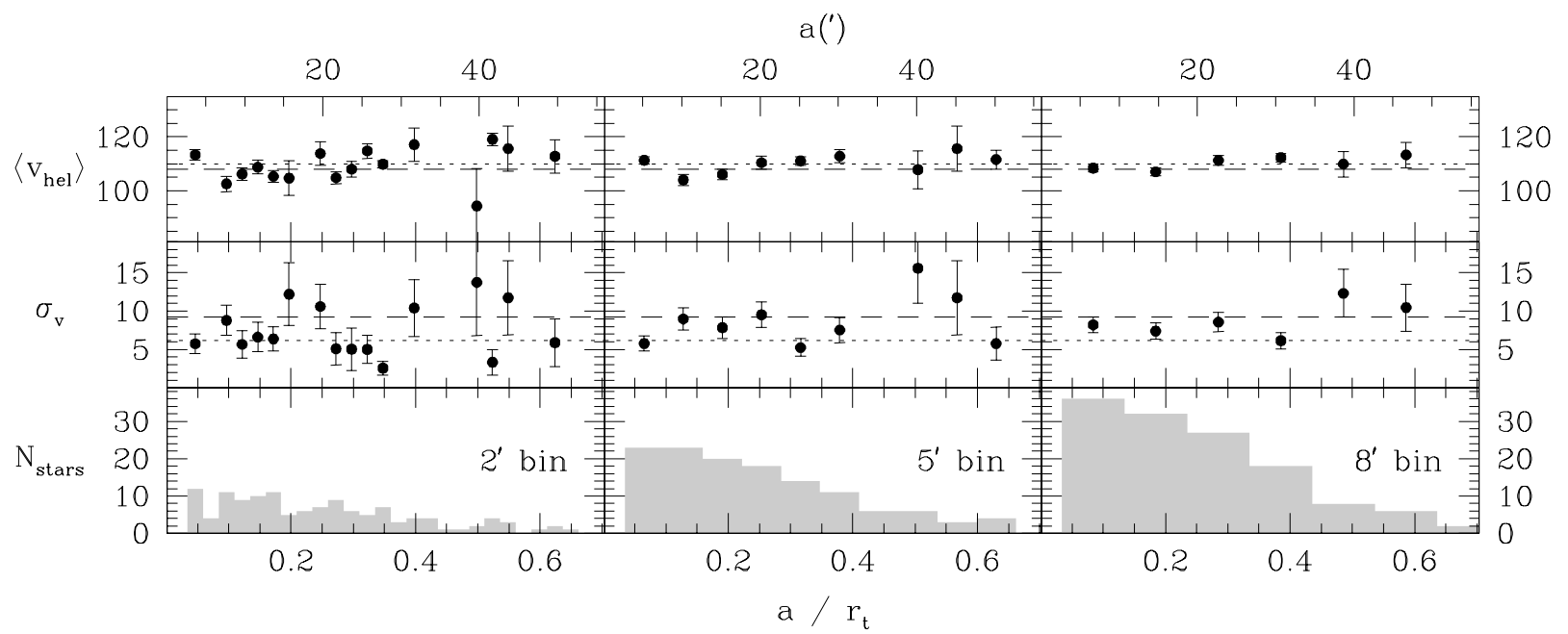

Fig. 12.- The azimuthally averaged $v_{\text {hel }}\left(\mathrm{km} \mathrm{s}^{-1}\right.$; top row), $\sigma_{v}\left(\mathrm{~km} \mathrm{~s}^{-1}\right.$; middle row), and number of stars per bin, $N_{\text {stars }}$, (bottom row) profiles. Points along the abscissa are marked both in terms of the semi-major axis distance, $a$, and the ratio of this distance to the mean of the derived King limiting radius found in $\S 5.2$. The results are given for three different bin sizes: $2^{\prime}$ (left column), $5^{\prime}$ (center column), and $8^{\prime}$ (right column). The values of $v_{\text {sys }}$ and the global $\sigma_{v}$ as derived by QDP (dotted line) and this study (dashed line) are also shown for reference. Note the value determined by QDP was determined from stars with $a \lesssim 10^{\prime}$; however, we extend the line here for comparison at all radii. 


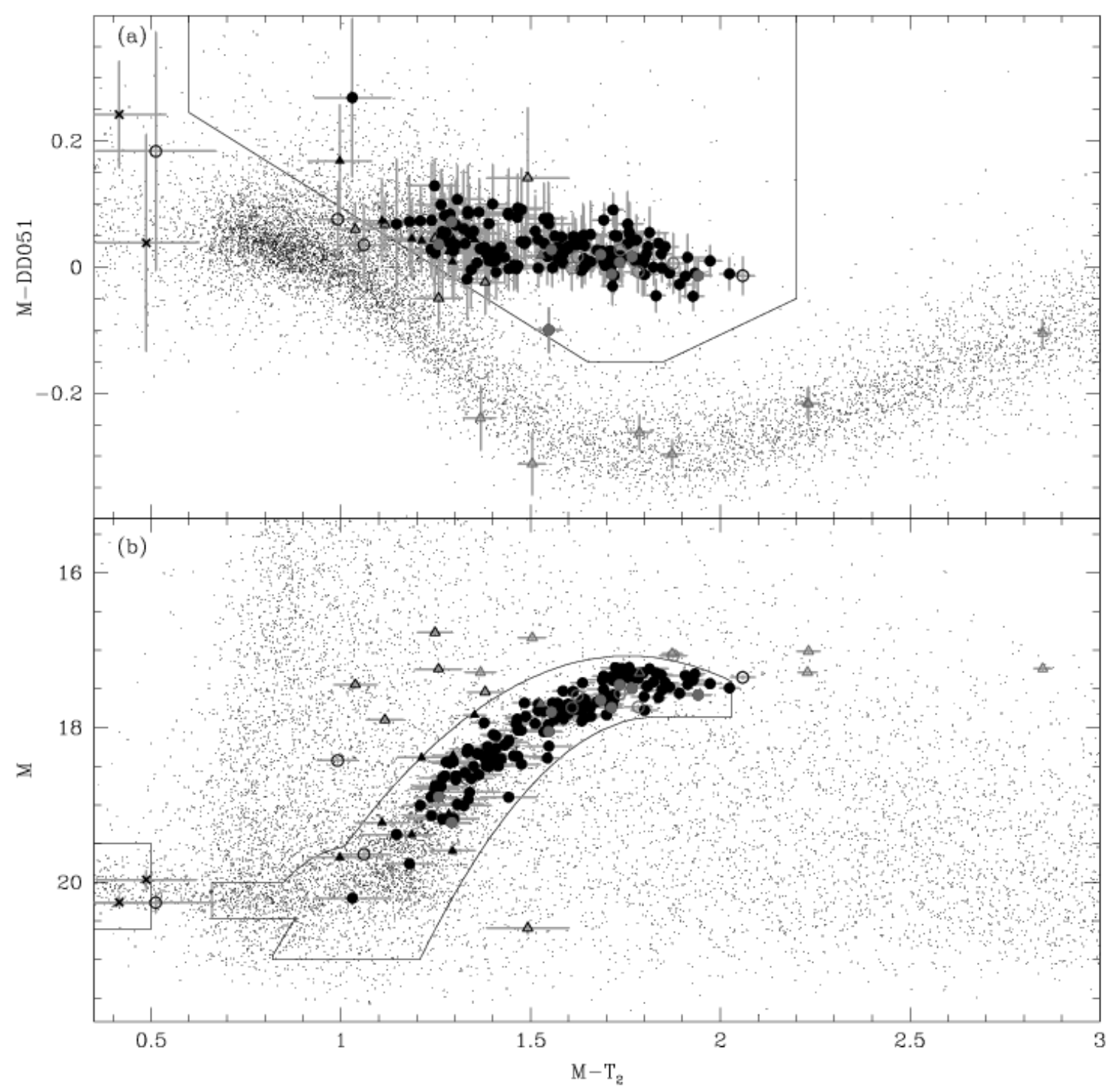

Fig. 13. - The distribution of all stars passing our photometric error and morphological limits in (a) the 2CD and (b) the CMD. The stars that have been observed spectroscopically are marked in the same way as Figure 11 and include error bars (grey). See Table 5 for a summary of the accuracy of our photometric selection technique as evidenced by our spectroscopic data. 


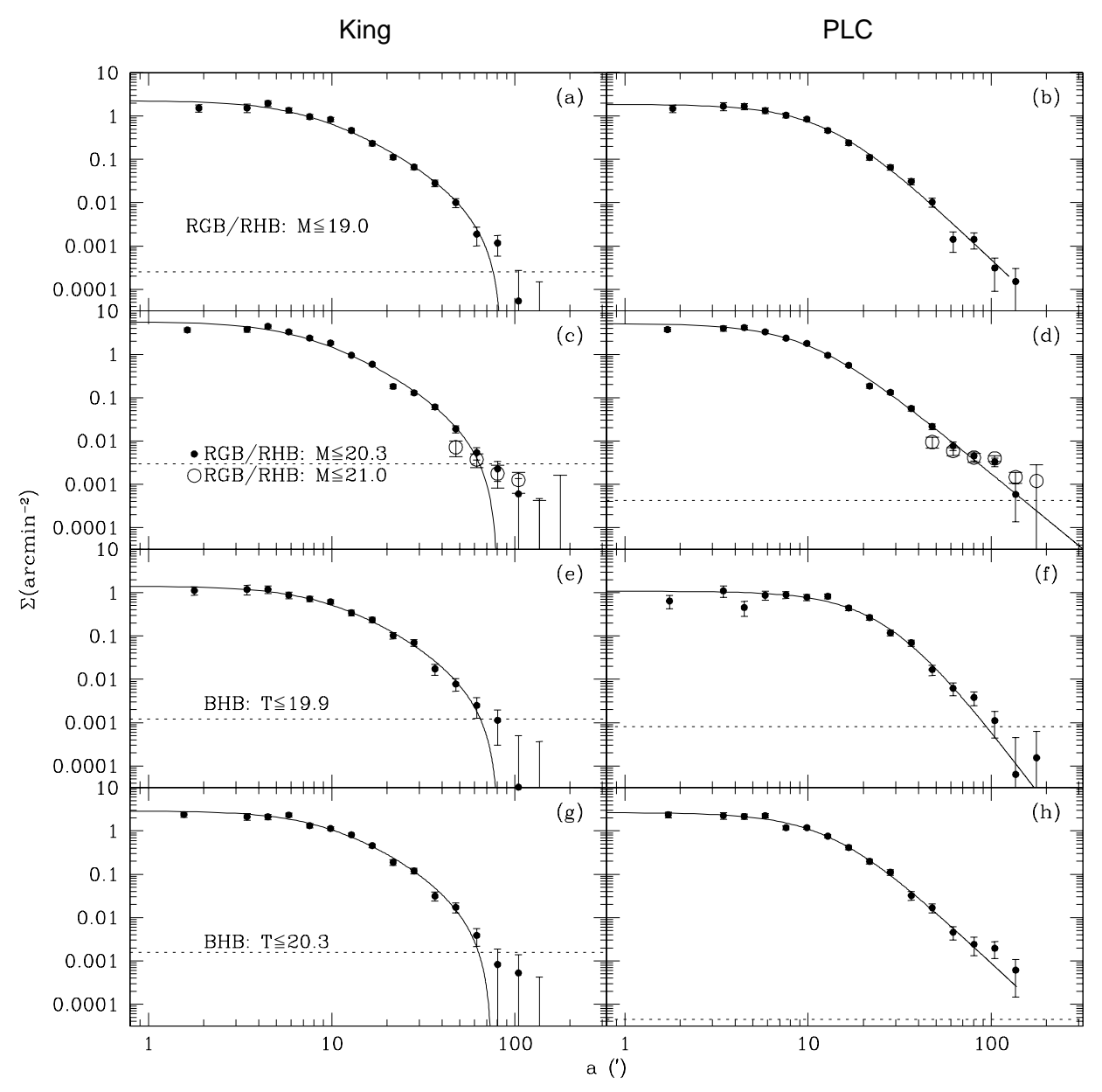

Fig. 14.- Initial radial surface density fits using the King (left) and PLC (right) models. The fits are shown for each sample: (a) and (b), the $M \leq 19.0$ sample; (c) and (d), the $M \leq 20.3$ and $M \leq 21.0$ samples; (e) and (f), the $T_{2} \leq 19.9$ sample; and (g) and (h), the $T_{2} \leq 20.3$ sample. The abscissa is given in units of the semi-major axis. The bestfitting models (solid lines) are overplotted on the resulting observed density points, which have error bars based on Poissonian statistics. Since our $M \leq 21.0$ sample did not contain the central parts of the galaxy, this sample is fit using model parameters derived for our $M \leq 20.3$ sample, scaled to densities appropriate for comparison (overplotted in panels (c) and (d) as open circles). (In detail, the $M \leq 21.0$ data points are scaled by the ratio of the number of stars in this sample to the number of stars in the $M \leq 20.3$ sample limited to the area covered by both samples.) The background densities as determined by our fitting program are subtracted from the observed density and overplotted (dotted line) in each panel to show where the $S / B=1$. The background for the $M \leq 21.0$ is taken to be the same as the $M \leq 20.3$ background, again scaled by the factor defined above (they are, therefore, equivalent on the plot). Note the significantly lower backgrounds determined by the PLC fits, whereas the King fits tend to place the background levels higher than the density of the break population. 


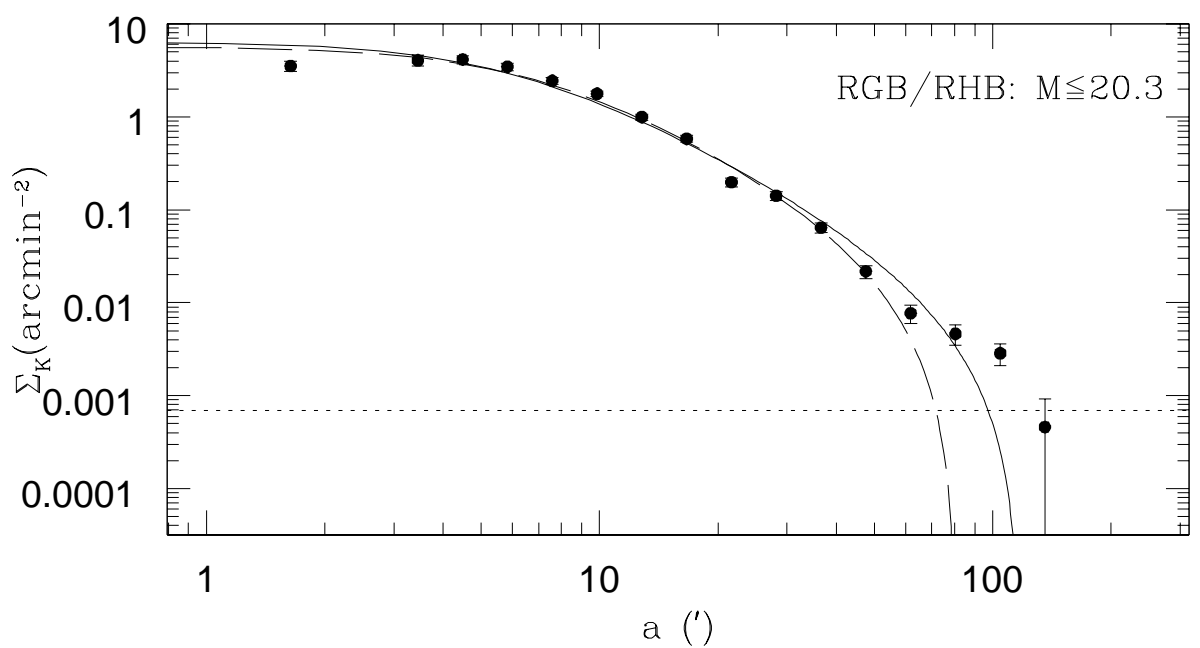

Fig. 15.- A demonstration of an attempt to fit our observed density distributions when fixing the background to the observed value. We plot the observed density distribution of the $M \leq 20.3$ sample that has been background subtracted according to the background density seen in our control fields (dotted line). The initial fit to this profile is shown (dashed line) along with the model derived after fixing the background density to our observed value (solid line). All parameters are nearly identical to those derived in the initial fit except for the King limiting radius which increases from $r_{\lim } \sim 80^{\prime}$ to $r_{\text {lim }} \sim 115^{\prime}$. 


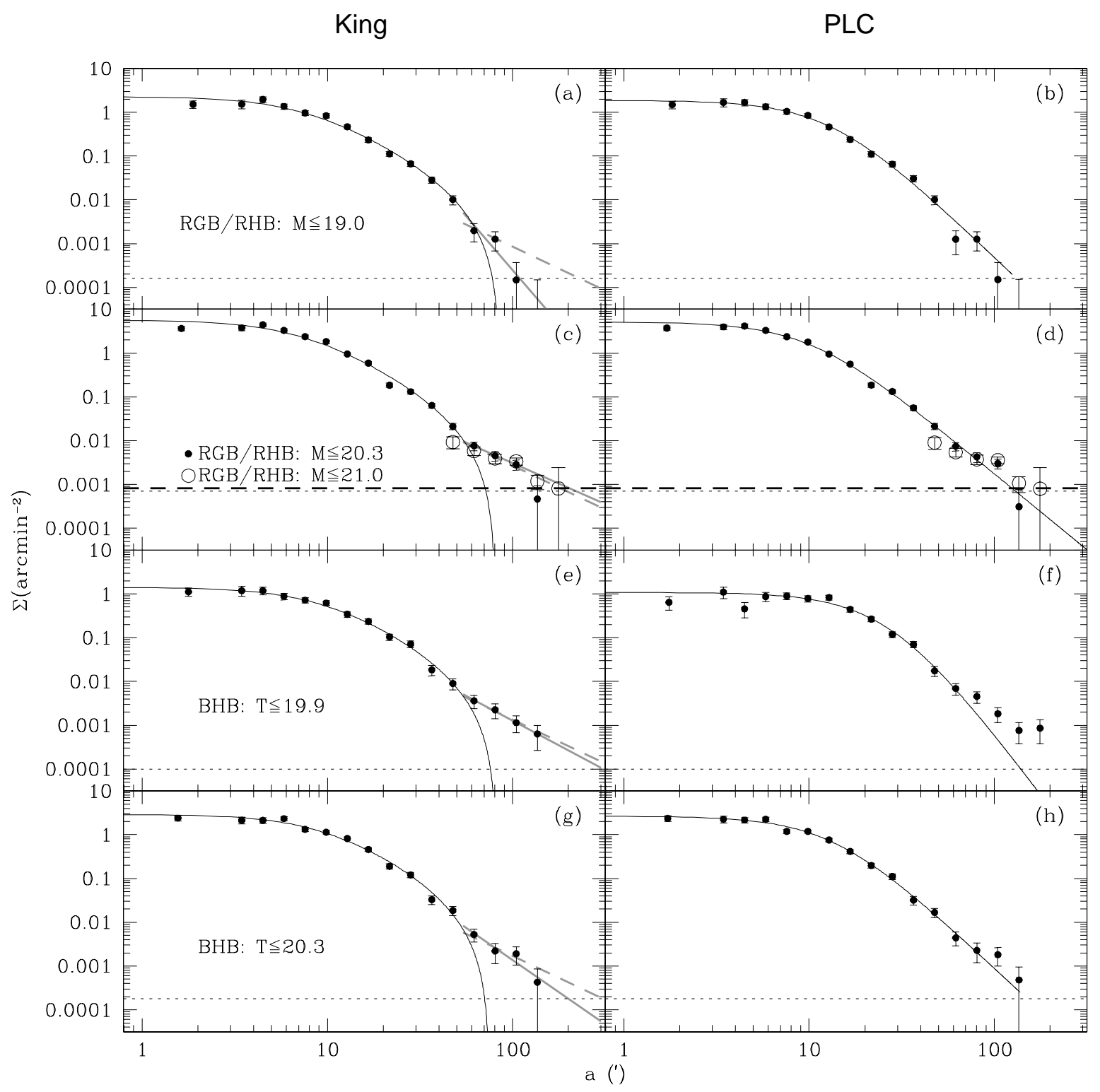

Fig. 16. - The same as Figure 14 except now the initial fits, which characterize the inner parts of Sculptor well, are compared to data corrected by our independently determined background levels. The background used is the larger of either $\Sigma_{\mathrm{CF}}$ or $\Sigma_{\mathrm{CMD}}$. Since we are now using backgrounds that are different between the $M \leq 20.3$ and $M \leq 21.0$ samples, both background levels are plotted in panels (c) and (d) (with dotted and dashed lines, respectively). Points considered to be part of the "break" population are modeled with a least-squares fit power law (solid grey line) in the left panels. The power laws are $\Sigma \propto r^{-4.9}$, $r^{-1.9}, r^{-2.3}$, and $r^{-2.9}$ in panels (a), (c), (e), and (g), respectively. We also show a simple $\Sigma \propto r^{-2}$ law (dashed grey line) for comparison. Goodness-of-fit values useful in comparing these fits are given in Table 11. 


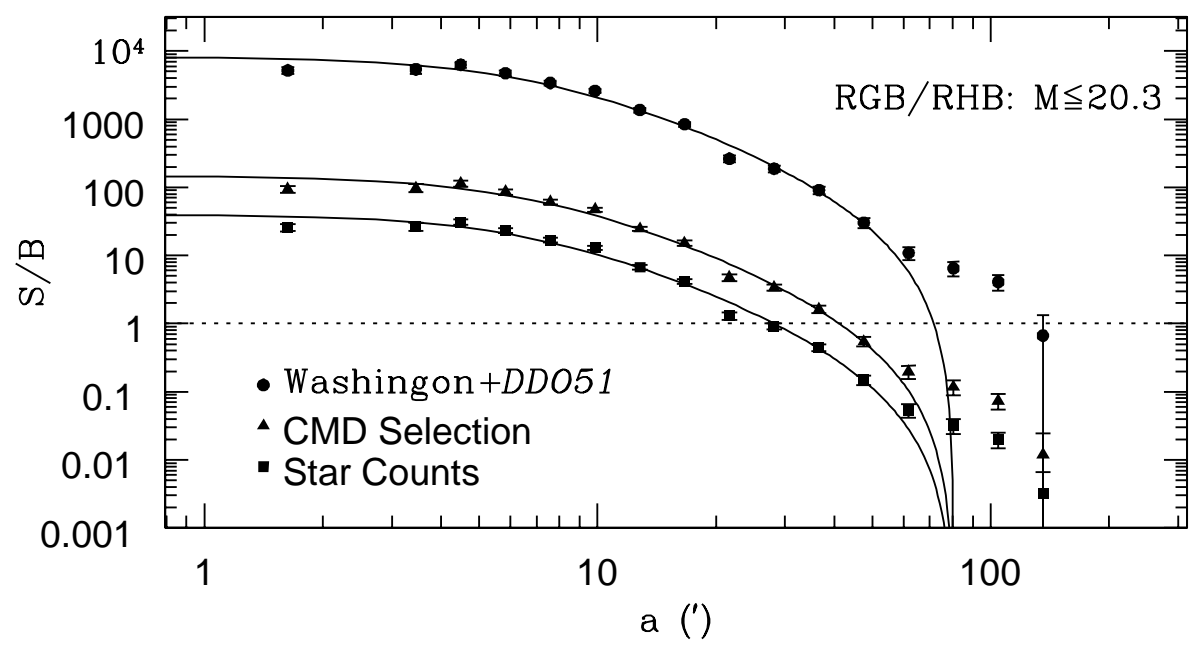

Fig. 17. - A comparison of $S / B$ for various strategies implemented to analyze our photometric catalog, including traditional starcounts (i.e., all stars within our error limits after removing galaxies; squares); a CMD-filtered scheme (making use of our CMD selection region from Figure 7; triangles); and our complete CMD and 2CD selection using Washington+DDO51 photometry (circles). The results for our $M \leq 20.3$ sample presented in Figure 16 are normalized to the expected $S / B$ for each of these three cases. This normalization is estimated by taking the ratio of the counts in the core of the dSph $\left(a<3^{\prime}\right)$ to those expected to be due to a background as measured by our control fields. Both the points and the model (solid line) are normalized by this ratio. As may be seen, the $S / B$ is improved by 0.6 order of magnitude in modifying the technique from simple starcounts to CMD-filtered starcounts, and improved by another 1.7 orders of magnitude in moving from CMD-filtered starcounts to our Washington+DDO51, CMD+2CD selection (§3). 


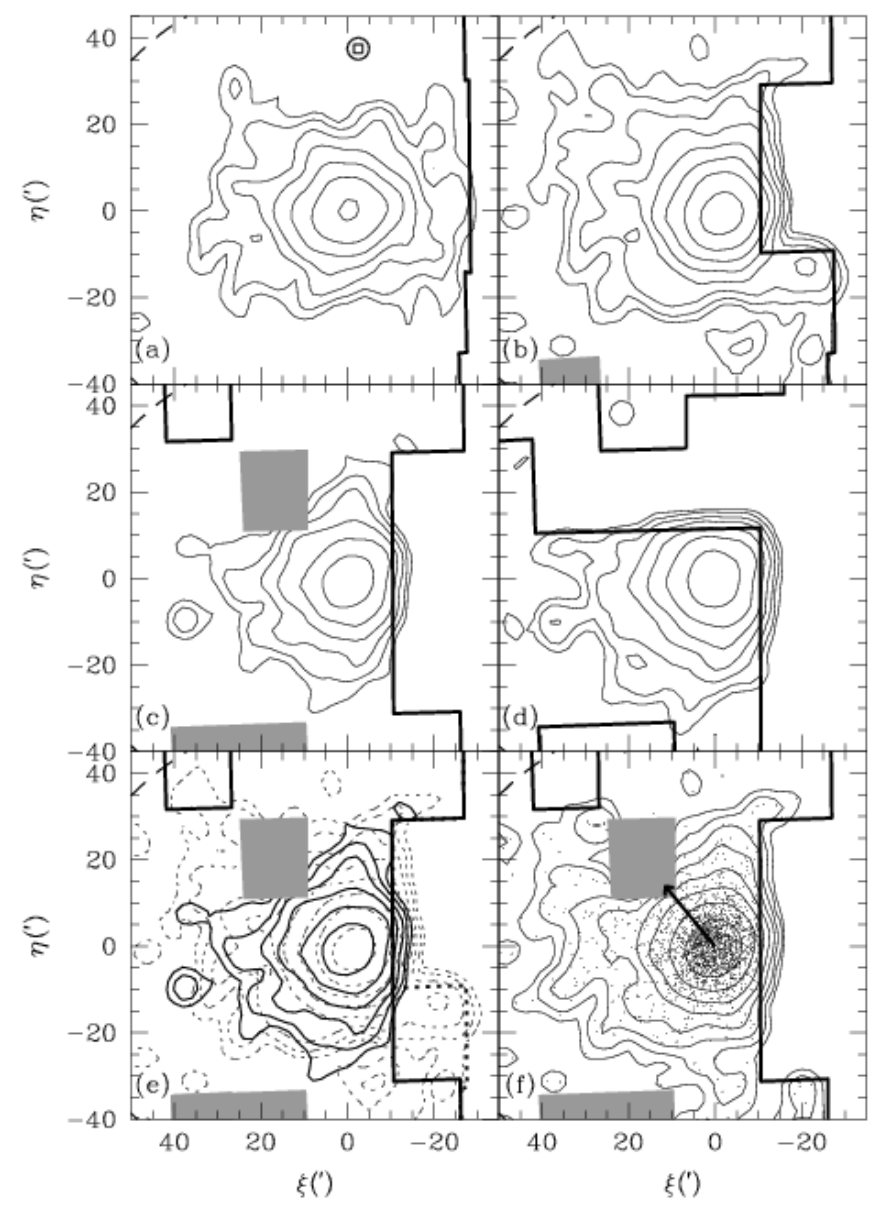

Fig. 18. - Contour plots of the (a) $M \leq 19.0$, (b) $M \leq 20.3$, (c) $T_{2} \leq 19.9$, and (d) $T_{2} \leq 20.3$ samples in the central regions of Sculptor. A section of the ellipse associated with the derived mean King limiting radius is also shown (dashed line to upper left). Panel (e) overlays the $M \leq 20.3$ (dotted lines) and $T_{2} \leq 19.9$ (solid lines) contours to show the strong correspondence between these two distributions. Panel (f) combines the two samples in (e) to give our most complete two-dimensional representation of Sculptor. In (f), we also plot the proper motion vector determined by Schweitzer et al. (1995), which has a position angle of $\sim 40^{\circ}$. The error in the position angle of the proper motion is indicated by the grey sector at the base of the arrow. Sections of the samples that are omitted due to insufficient depth are displayed with grey boxes as in Figure 10. For all contours, individual stars have been smoothed by a unit-normalized Gaussian kernel with $\sigma=2.5$, truncated at $3 \sigma$. The one $\sigma$ extent of this kernel and the sampling bin $\left(2^{\prime} \times 2^{\prime}\right)$ size are shown at the top of (a). The relative density levels are the same for each panel: 1, 2, 4, 8, 16, 32, 64 and 128. For a sense of what this translates to in terms of the actual star sample, the stars used to create the contours in (f) are over-plotted. 

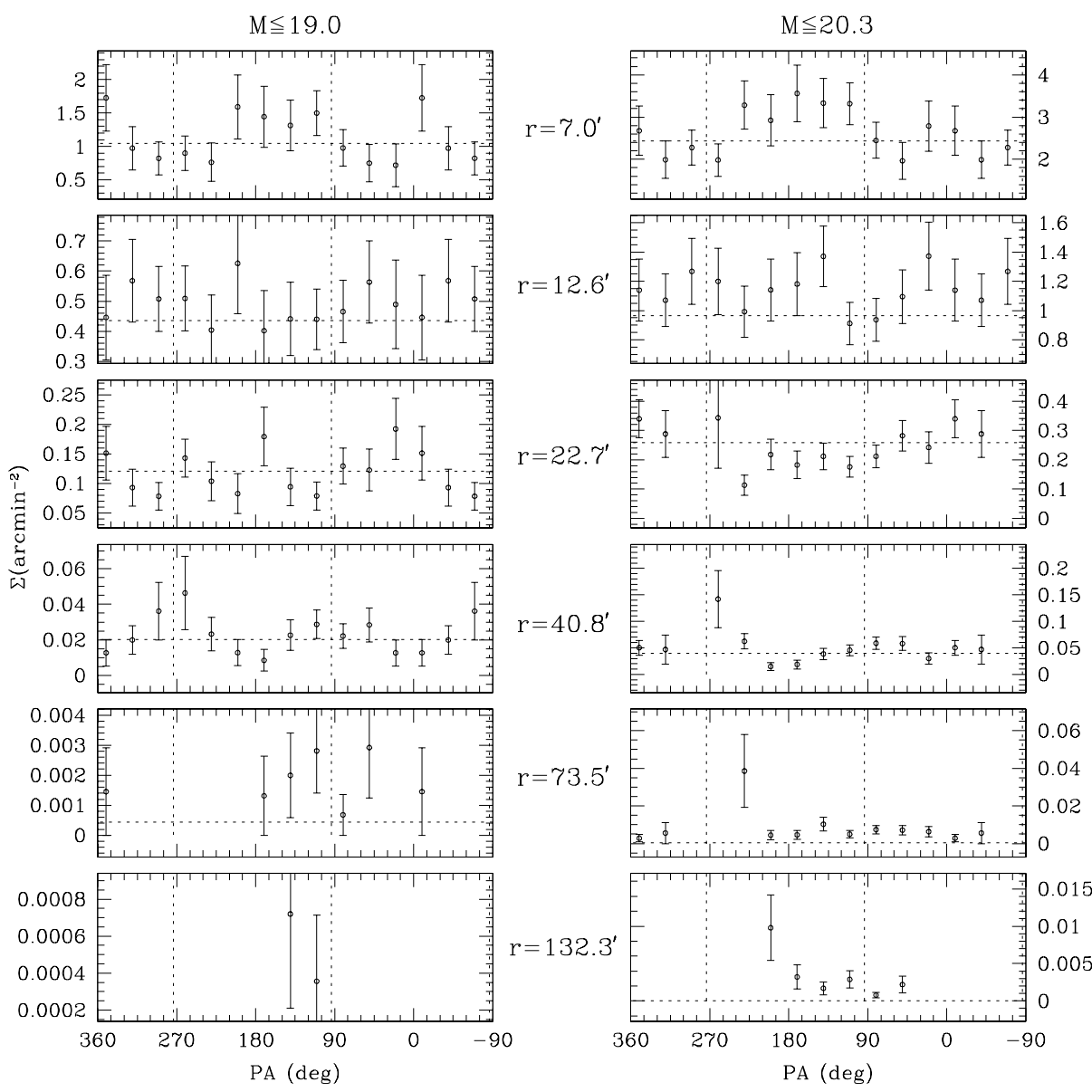

Fig. 19. - Measures of the variation in the surface density with azimuth in the $M \leq 19.0$ (left) and $M \leq 20.3$ (right) samples as a function of semi-major axis radius. The marked radii are the bin centers just as in previous radial plots. The horizontal dotted line shows for each elliptical annulus the density for that annulus derived from the global King profile fit to all radii. Were there no variation in isophote shape with radius, the densities of individual annular sectors would be constant at all radii, and this constant value should match that of the global fit, to the degree that the fitted radial profile is a good match to the observed radial profile. Changes in isophote shape from the nominal Sculptor ellipticity (line 5 of Table 9) will show up as systematic deviations in the densities of annular sectors at any radius. The tendency for systematic deficits in density near the semi-major axes (vertical dotted lines) and larger densities near the semi-minor axes seen at smaller radii indicate that the central Sculptor profile is rounder than the nominal ellipticity from the global fit. On the other hand, the opposite trend observed at larger radii (e.g., at $r=40$ '8) suggests that the outer contours of Sculptor are more elliptical than the global King profile fit, with the ellipticity along the same $P A$ as the global fit. No strong evidence for isophotal twisting is seen, but incomplete survey coverage at intermediate and large radii leaves great uncertainty in this conclusion. 


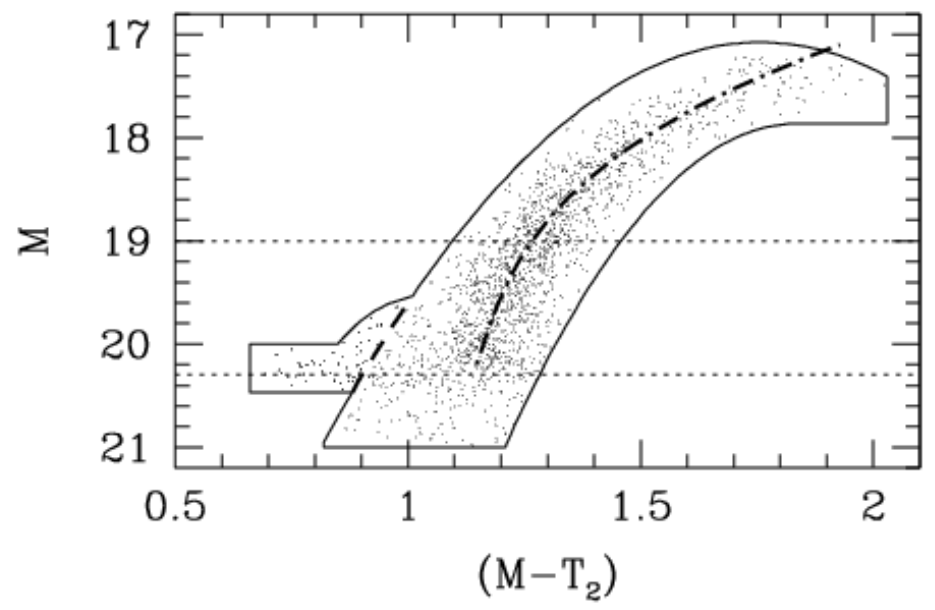

Fig. 20.- Demonstration of our division of the Sculptor RGB into "metal-poor" (blue) and "metal-rich" (red) halves. A fourth-order polynomial has been fit to the distribution of stars within our RGB/RHB selection (dot-dashed line). This division of the RGB into blue and red parts is done only for the $M \leq 19.9$ and $M \leq 20.3$ samples whose limits are shown by dotted lines. Because the RHB population is expected to track the spatial distribution of the red half of the RGB, we trim (with the dashed line) the RHB-dominated part of our selection region away from the blue half of the $\mathrm{RGB}$. This will reduce, but not totally eliminate the "dilution" of the distinct blue RGB spatial distribution due to the the overlap of the RHB with the blue RGB in the CMD (e.g., Figure 7). 


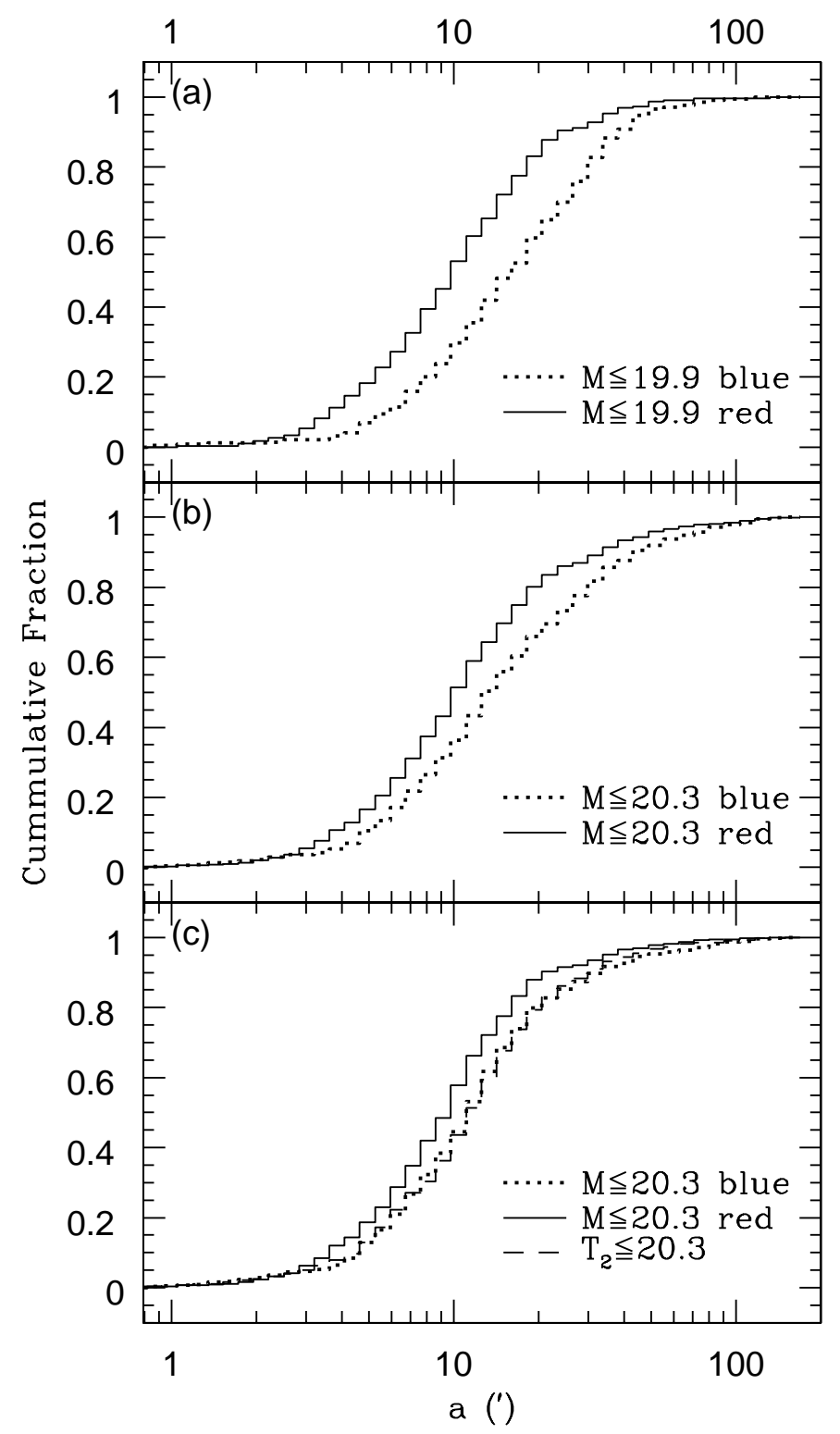

Fig. 21.-Cumulative distributions of the red (solid lines) and blue (dotted lines) populations (see Figure 20) for (a) the $M \leq 19.0$ sample, (b) the $M \leq 20.3$ sample, and (c) the $M \leq 20.3$ sample only over the area corresponding to the $T \leq 20.3$ sample (dashed line). In all three cases the red population is much more concentrated than the blue population. In (c), the BHB population is shown to closely follow the blue $M \leq 20.3$ sample. A similar test could be done for the RHB, which should follow the red population; however, we are unable select a reasonably pure sample of RHB stars from our CMD with a large enough sample size. 


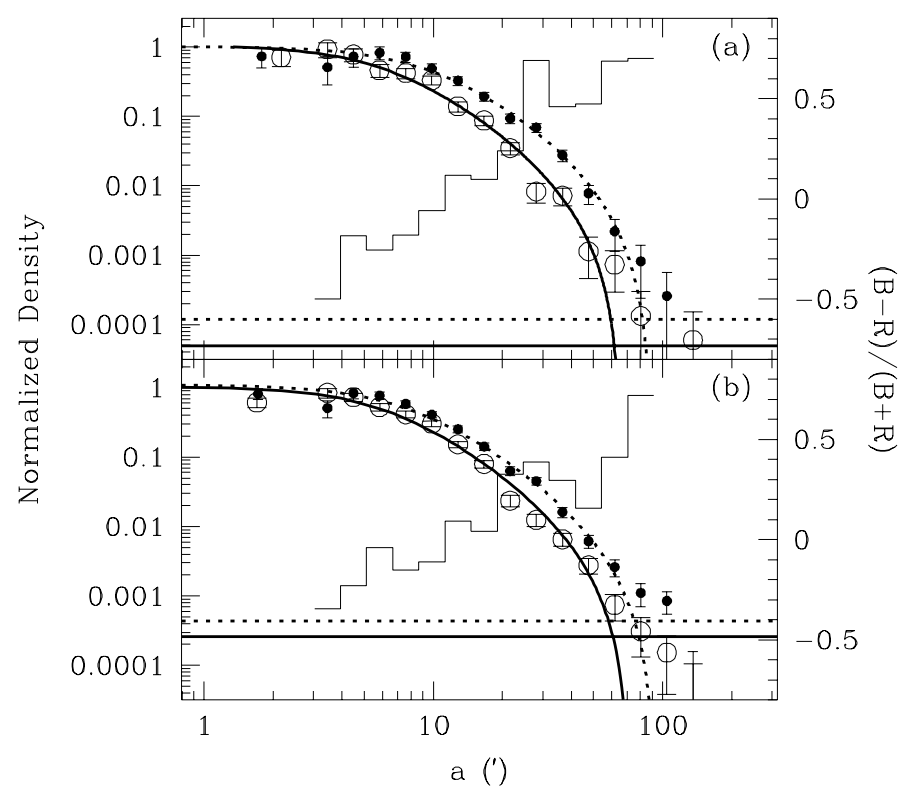

Fig. 22.- Radial surface density distributions for the blue (filled circles) and red (open circles) RGB populations in the (a) $M \leq 19.0$ and (b) $M \leq 20.3$ samples. The best-fitted King profiles are overplotted for the blue (dotted line) and red (solid line) populations along with the background determined by the "CMD offset" method. As illustrated in Figure 21, the blue population is much more extended than its red counterpart. This gradient is further demonstrated by the overlayed color index $(B-R) /(B+R)$ (shown by the histogram), where $B$ and $R$ are, respectively, the number of "blue" and "red" stars within a given annulus. 


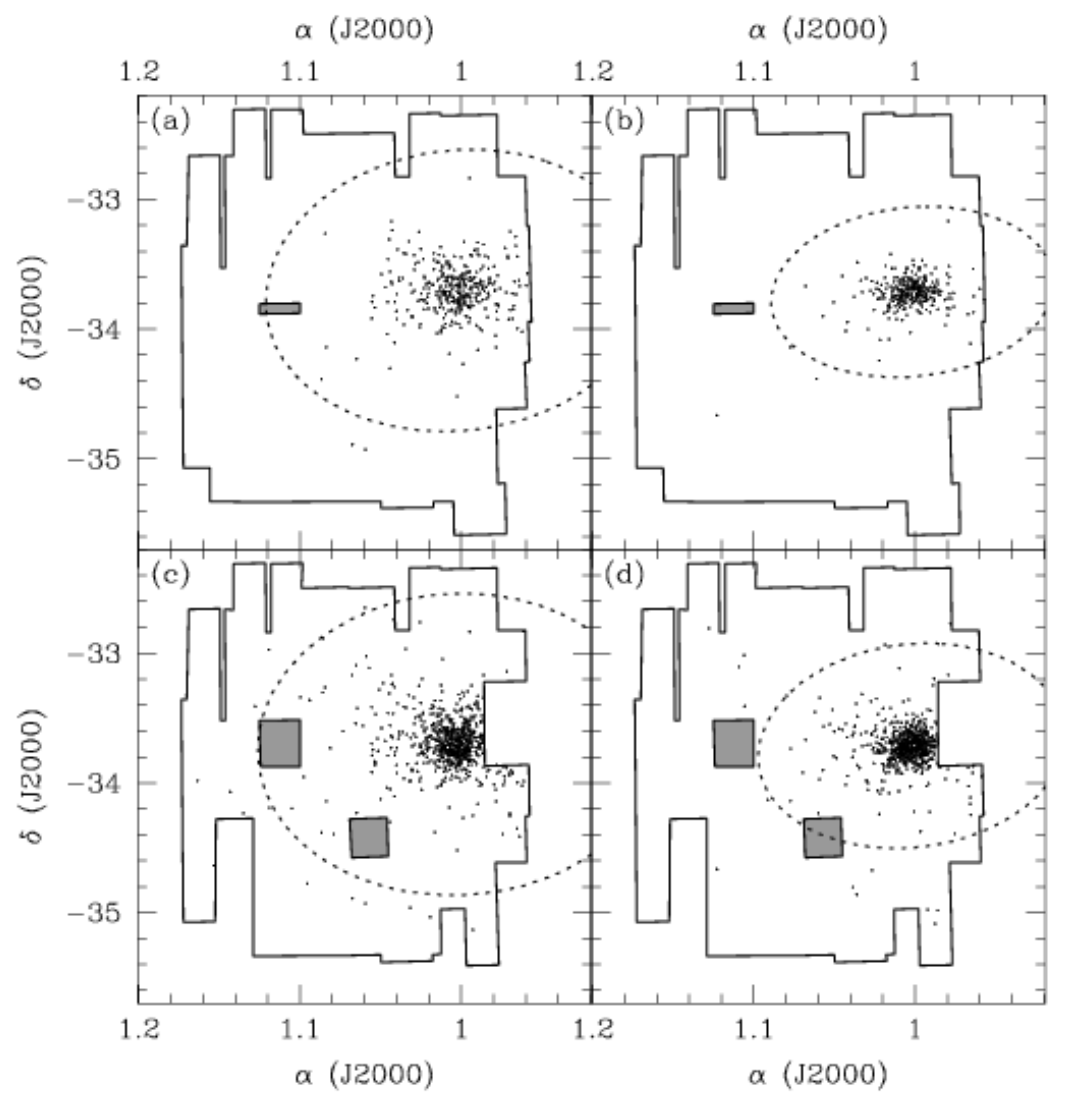

Fig. 23. - The spatial distribution of the "blue" (left) and "red" (right) RGB populations for the $M \leq 19.0$ (top) and $M \leq 20.3$ (bottom) samples. The derived King limiting radii (dotted line) are also shown. The red population is much more concentrated than the blue population; but, interestingly, it is also more elliptical. 